\title{
Measurements of Magnetic Fiber Dynamics in Magnetic Fields using Optical and Electrical Techniques
}

by

Shaun Swan

A thesis

submitted to the Victoria University of Wellington in fulfilment of the requirements for the degree of

Master of Science

in Engineering.

Victoria University of Wellington

2019 



\begin{abstract}
The fabrication of piezoelectric ceramics (Piezoceramics) currently relies on a costly dice and fill process to create an array of aligned pillars. These pillars act as waveguides, improving the performance of the piezoceramic wafers over the bulk piezoceramic alone. It is theorised the creation of aligned pores in the piezoceramic may exhibit the same waveguiding effect, removing the need for the dice and fill process.

A technique for creating these pores is in development at Callaghan Innovation, New Zealand, where nickel coated carbon fibers are added to the ceramic slurry, aligned with a magnetic field, and attracted to the bottom of a mold. The number of fibers and degree of alignment dictate the waveguiding effectiveness and hence the performance of the piezoceramic. Additionally the time taken for fibers to form an array in the bottom of the mold dictate the piezoceramics fabrication time. Thus it is crucial to be able to measure the alignment and magnetically assisted sedimentation of these fibers in-situ. However the ceramic slurry is opaque, hence the optical methods traditionally can not be implemented.

This thesis describes the development and implementation of an electrical technique using the anisotropic conductance of fibers, for measuring fiber dynamics during the fabrication of piezoceramics. The results of this electrical technique are compared to both optical monitoring results in a transparent solution, and models for the motion of rigid cylinders in a fluid suspension.

The change in conductance corresponding to fiber rotation was found to have a time constant corresponding to fiber rotation which is a scalar multiple of that of transmission microscopy and the mathematical modeling. This is a product of the geometry of the electrode configurations used to measure conductance. Furthermore, for fiber rotation, the fiber concentration in the solution
\end{abstract}


changes the effective fluid viscosity due to hydrodynamic turbulence created by the rotating fibers.

The conductance change corresponding to the magnetically assisted fiber settling is in good accordance with both the optical observations and mathematical modeling for $50 \mathrm{mPas}$ solutions, however for $30 \mathrm{mPas}$ solutions the modeling underestimates the settling time by $20 \%$. The maximum fiber concentration to create a single layer of aligned fibers in the bottom of the mold was found to be 12 fibers $/ \mathrm{mm}^{3}$. Exceeding this limit results in a secondary and tertiary layer of fibers forming directly below the fiber suspension injection location. 


\section{Acknowledgments}

I would like to thank Gideon Gouws of Victoria University and Mike Staines of the Robinson Research Institute for being my supervisors during the completion of this thesis.

I would also like to thank Simon Granville of the Robinson Research Institute for assisting with SQUID measurements, and Sarah Spencer of the Robinson Research Institute for assisting with the SEM analysis components of the project.

Finally, I would like to thank Trent Little for the many hours of discussions we have had in the lab. Without these discussions working late nights would have been impossible. 



\section{Contents}

1 Introduction 1

2 Background 5

2.1 Magnetism ........................ 5

2.1 .1 Diamagnetism . . . . . . . . . . . . . 6

2.1 .2 Paramagnetism . . . . . . . . . . . . 8

2.1 .3 Ferromagentism . . . . . . . . . . . . . . 9 9

2.2 Synthesis and Implenetation of Magnetic Particles . . . . . . . . 13

2.2.1 Manipulation of Magnetic Particles . . . . . . . . . . . 15

2.2.2 In Situ Monitoring of Magnetic Particles . . . . . . . . . . 18

2.3 Fabrication of Porous Ceramics . . . . . . . . . . . . . . . 22

2.3.1 Forces of a Fiber During Ceramic Fabrication . . . . . . . . 22

2.4 Literature Review of Magnetic Alignment and Sedimentation of Slender Bodies . . . . . . . . . . . . . . . . 26

3 Experimental Methodologies 31

3.1 Selection and Properties of Magnetic Fibers . . . . . . . . . . . . 31

3.2 Selection of a Suspension Fluid . . . . . . . . . . . . . . . 38

3.3 Experiment Setup for the Electrical Measurement of Fiber Translation and Rotation . . . . . . . . . . . . . . . . . 40

3.3.1 Lock-in Amplifier . . . . . . . . . . . . . . . . . . . 42

3.3 .2 Multiplexor ................... 44

3.3.3 Electrode configurations . . . . . . . . . . . . . 44 
3.3.4 Magnetic Cells . . . . . . . . . . . . . . . . 48

3.3 .5 Solenoid . . . . . . . . . . . . . . 50

3.3.6 Labview Software . . . . . . . . . . . . . 50

3.4 Experimental Setup for the Optical Measurement of Fiber Translation and Rotation . . . . . . . . . . . . . . . . 54

3.4.1 Helmholtz Coil . . . . . . . . . . . . . . . . . . 55

3.4 .2 Basler Camera . . . . . . . . . . . . . . . 55

3.4.3 Transparent Fiber Mold . . . . . . . . . . . . . 58

3.5 Experimental procedures . . . . . . . . . . . . . . . 59

3.5.1 Fiber Suspension Preparation . . . . . . . . . . . . . 59

3.5.2 Electrical Experiments . . . . . . . . . . . . . 60

3.5.3 Optical Experiments . . . . . . . . . . . . . 62

4 Fiber Rotation $\quad 65$

4.1 Helmholtz coil . . . . . . . . . . . . . . . . . . 66

4.2 Mathematical Modeling of Fiber Rotation . . . . . . . . . . . . 69

4.2.1 Single Fiber Modeling . . . . . . . . . . . . . . 71

4.2.2 Monte-Carlo Simulation . . . . . . . . . . . . . . . 71

4.3 Results of Fiber Rotation using Electrical Techniques . . . . . . . . 75

4.3.1 Heating in the solenoid . . . . . . . . . . 75

4.3 .2 Fiber Relaxation . . . . . . . . . . . . . . . 83

4.4 Quadrant Electrode Configuration ... . . . . . . . . . 84

4.5 Ring Electrode Configuration . . . . . . . . . . . . . . . . 86

4.6 Discussion . . . . . . . . . . . . . . . . 88

5 Fiber Translation $\quad 95$

5.1 Modeling of the Magnetically Assisted Sedimentation of Fibers . 95

5.2 Visual Observations of Fiber Translation . . . . . . . . . . . . . . . 110

5.3 Electrical Measurement of Fiber Translation . . . . . . . . . . . . . 112

5.4 Discussion . . . . . . . . . . . . . . . . . . 116 
CONTENTS

6 Summery and Conclusion 119

6.1 Recommendations for the Fabrication of Porous Ceramics . . . 120

6.2 Future Work . . . . . . . . . . . . . . . . . . . . . 121 


\section{List of Figures}

1.1 The "dice and fill" method for creating piezoceramic wafers. . . . 3

2.1 Pyrolytic carbon levitation due to a strong magnetic repulsion . . 7

2.2 The magnetic saturation curve for paramagnetic materials . . . 9

2.3 Moving domain walls in a ferromagnetic material . . . . . . . 11

2.4 The magnetic saturation curve for ferromagnetic materials . . . . 12

2.5 Alignment of superparamagnetic material coated graphite flakes. 16

2.6 Magnetic drug delivery in cells. . . . . . . . . . . . . . . 17

2.7 A schematic representation of a magnetic particle imaging system 19

2.8 An experimental magnetic particle manipulation system consisting of orthogonal coils. . . . . . . . . . . . . . . . . . 21

2.9 The process for creating porous ceramics under development at Callaghan Innovation . . . . . . . . . . . . . . . 23

3.1 Chemical vapor deposition results in a high quality finish compared to electroplating . . . . . . . . . . . . . . 33

3.2 Fiber length distribution of the nickel coated carbon fibers. . . . . 34

3.3 SEM photography of the carbon fibers showing imperfections and damage in the nickel coating. . . . . . . . . . . . . . . 35

3.4 Magnetic hysteresis curve of nickel coated carbon fibers . . . . . 36

3.5 Processing steps for preparing the bulk fibers to an experimentally ready state . . . . . . . . . . . . . . . . . . . 39

3.6 High level block diagram of the experimental setup . . . . . . . 43

3.7 Model and photograph of the quadrant electrode configuration . 46 
3.8 Model and photograph of the ring electrode . . . . . . . . . . . . 47

3.9 Photograph of the ladder electrode configuration and the corresponding pairs used for sensing applications. . . . . . . . . . . 49

3.10 The open magnetic cell allows optical access whereas the enclosed magnetic cell maximises magnetic flux though the piezoceramic mold. . . . . . . . . . . . . . . . . . . . . . . . 51

3.11 The magnetic field strengths in both the open and enclosed cell . 52

3.12 The magnetic field gradients in both the open and enclosed cell . 53

3.13 Photograph of the Helmholtz coil used in optical experiments . . 56

3.14 Helmholtz coil field strength-applied current relationship . . . . . 57

3.15 The Basler camera used for optical experiments . . . . . . . . . . 58

3.16 Agitating the fiber suspension to uniformly distribute fibers results in air bubbles in the fluid which are removed thorough the

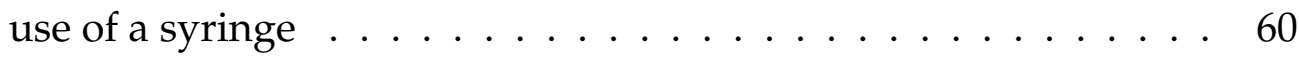

4.1 Optical monitoring of the fiber rotation for a single fiber in a Helmholtz coil generated magnetic field . . . . . . . . . . . 66

4.2 Fiber rotation in a viscous solution is dependent fiber length, fluid viscosity, and initial orientation . . . . . . . . . . . 68

4.3 The effects of initial fiber orientation on the modeling a single fiber subject to a magnetic field. . . . . . . . . . . . . 72

4.4 The effects of viscosity on the modeling of a single fiber in a glycerol water solution subject to a magnetic field. . . . . . . . . 73

4.5 A comparison between the analytical model and the optical measurements. . . . . . . . . . . . . . . . . 74

4.6 Monte Carlo simulation of the net fiber angle. . . . . . . . . . . 76

4.7 A comparison between the time constant of heating in the solenoid and the fiber rotation time constant . . . . . . . . 78

4.8 Conductance temperature relationship for 75 weight percent glycerol water solutions . . . . . . . . . . . . . 79

4.9 Temperature adjusted change in conductance for fibers rotating in the solenoid induced magnetic field . . . . . . . . . . . . . . 81 
4.10 The change in conductivity between randomly oriented and aligned fibers. . . . . . . . . . . . . . . . . 82

4.11 Relaxation in the fiber orientation after the removal of the magnetic field. . . . . . . . . . . . . . . . . . . . 84

4.12 Fiber rotation as measured by the quadrant electrode configuration 85

4.13 Time constants for varying fiber concentrations measured using the quadrant electrode. . . . . . . . . . . . . . . . . . 87

4.14 Time constants for varying fluid viscosities measured using the quadrant electrodes. . . . . . . . . . . . . . . 88

4.15 Raw measurements of the change in conductance from the ring electrode configuration . . . . . . . . . . . . . . . . . . 89

4.16 The time constant of fiber rotation as measured by the ring electrode configuration with varying fiber concentrations . . . . . . 90

4.17 An increase in viscosity results in an increase in the conductance time constant measured with the ring electrode configuration . . 91

4.18 A comparison of conductance time constants with an applied field and optically obtained time constants of fiber rotation . . . . 93

5.1 Magnetic field gradients used in the modeling of the fiber motion 98

5.2 Modeled position profile of fibers during sedimentation . . . . . 100

5.3 Modeled velocity profile of fibers during sedimentation . . . . . . 101

5.4 Modeled acceleration profile of fibers during sedimentation . . 102

5.5 Modeled magnetic force profile of fibers during sedimentation . 103

5.6 Modeled drag force profile of fibers during sedimentation . . . 104

5.7 Modeled sedimentation time as a function of fluid viscosity for the open magnetic cell . . . . . . . . . . . . . . . . . . 105

5.8 Modeled sedimentation time as a function of fluid viscosity after adjustments are made for the practical design of the magnetic cells 106

5.9 The motion of fibers in the enclosed magnetic cell as a function of initial position . . . . . . . . . . . . . . . . 108

5.10 Simulated response from the measurement system . . . . . . . 109

5.11 Visual observations of the setting time . . . . . . . . . . 111 
5.12 Multiple layers of fibers are observed to form for high fiber concentrations . . . . . . . . . . . . . . . . . 112

5.13 The negative field gradient results in fibers collecting in the top of the cell . . . . . . . . . . . . . . . . . . . . . . . 113

5.14 Measurements of fiber motion using the electrode configuration over a short time span . . . . . . . . . . . . . . . . . 114

5.15 Measurements of fiber motion using the electrode configuration over a long time span . . . . . . . . . . . . . . . . 115 


\section{List of Tables}

3.1 Summery of the properties of nickel coated carbon fibers . . . . 37

3.2 Viscosity of Glycerol-water solutions as a function of temperature 41

3.3 The time current was applied to the solenoid was dependent on the magnitude of the current in order to mitigate thermal effects. 61

4.1 The time constant for fibers suspended in various glycerol-water solutions using a Helmholtz coil in conjunction with video analysis 69

4.2 The effects of remnant magnetisation in the fibers results in a small change in the time constant of rotation. . . . . . . . . . 83

5.1 Constants used in the fiber translation modeling. . . . . . . . . . . 97

5.2 Time taken for fibers to completely settle to the bottom of the mold in the magnetic cell. . . . . . . . . . . . . . . . . . 117 


\section{Chapter 1}

\section{Introduction}

Magnetic nano- and microparticles have become an area of interest due to their range of potential technological, medical, and environmental applications. This is due to the wide range of properties these materials can exhibit. For example, microparticles with a stable, switchable magnetic state can be used to represent bits of information in data storage applications [1]. Particles which exhibit superparamagnetic ${ }^{1}$ properties, and are stable in both a water and physiological environment, can be used for the targeted treatment of cancerous tumors [2]. Polymeric magnetic particles which bind to specific bacteria, or whole bacteria species, can be used in the clinical analysis of drinking water in order to evaluate its safety and microbial quality [3].

On a slightly larger scale, magnetic microwires can be added to a composite in order to add anisotropic properties. These range from field/stress tunable properties [4] [5] which have been suggested for use in stress sensitive media for remote non-destructive health monitoring of structures, and microwave absorption [6] [7] for use in aircraft coatings, whist maintaining the composites mechanical performance. Fibers used for these applications typically have diameters on the order of microns with an aspect ratio greater than 100.

While the specific applications for these materials are diverse, they share

\footnotetext{
${ }^{1}$ Superparamagnetic particles have a single magnetic domain and hence have no magnetic hysteresis.
} 
a common property in the ability to manipulate the position, orientation, and electrical properties of these particles using magnetic fields. For example, magnetic memory such as magnetic random access memory (MRAM) relies on the magnetic manipulation of the materials electrical resistance through changing electron spin states [8]. Anisotropic properties can be created through the use of linear magnetic fields to align magnetic particles in a particular direction. Finally, magnetic field gradients can be used to direct particles to a target location.

A potential new application of these magnetic microwires is in the fabrication of porous piezoelectric ceramic (piezoceramic) transducers [9]. A piezoelectric material is one which expands and contracts with the application of a strong electric field. This expansion and contraction leads to the production of ultrasonic waves, however the bulk material has a large amount of mechanical loss [10]. Currently, the mechanical loss is reduced through the fabrication of bulk piezoceramic wafers which are processed using a so called "dice and fill" mechanical technique [11]. This is illustrated in Figure 1.1, where the fabrication steps to produce an array of $50 \mu \mathrm{m}$ piezoelectric pillars are demonstrated. The resulting pillars promote the expansion of the piezoelectric perpendicular to the surface, acting as a waveguide for the ultrasonic waves. The precision with which this mechanical step can be performed not only limits the imaging resolution and performance, but also increases transducer costs roughly 100-fold. This mechanical fabrication step is the key element imposing a major barrier to the wider application of ultrasonic technology.

It is hypothesized that the waveguiding effect in piezoceramics can also be achieved by the controlled presence of uni-axially oriented pores in the material, eliminating the need for a costly dice and fill process. The development of such a novel piezoceramic is currently under development by Callaghan Innovation, New Zealand. To produce such a piezoceramic containing a high density of aligned pores, a process is being developed where magnetic fibers are added to the ceramic slurry. This slurry is then cast to produce $1-2 \mathrm{~mm}$ thick ceramic wafers. In order to impart the desired anisotropic structure to these wafers, the magnetic fibers must be manipulated and aligned by an external magnetic field 


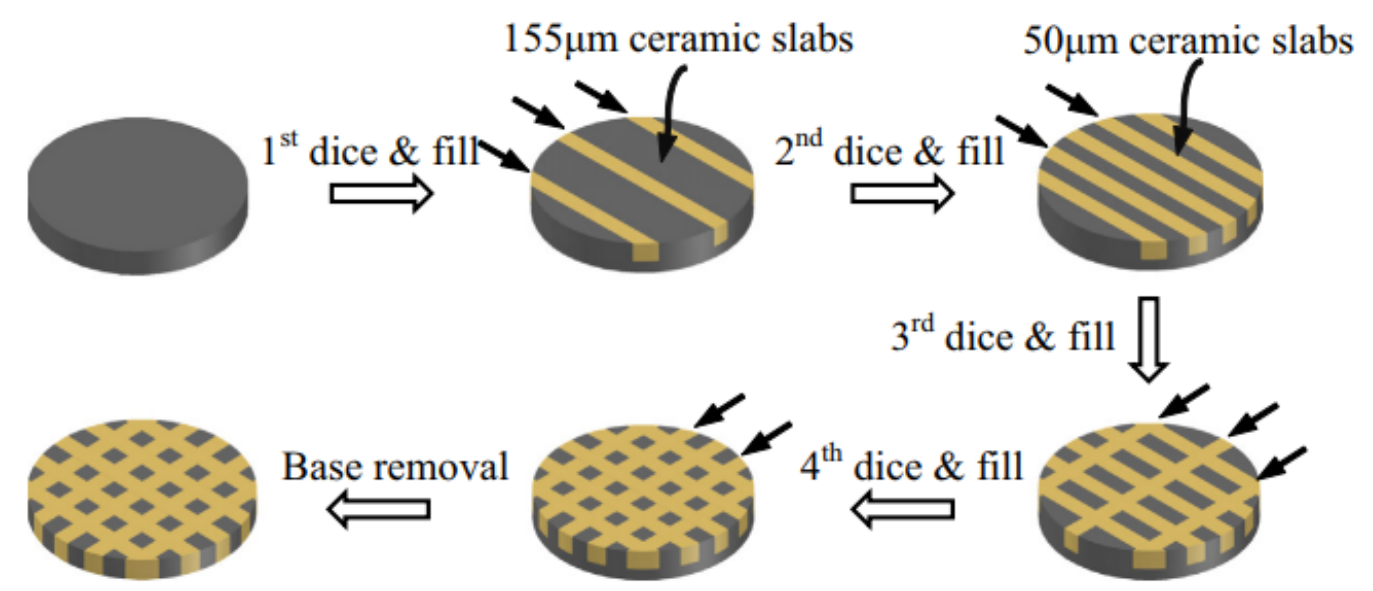

Figure 1.1: The "dice and fill" method requires making multiple precision cuts in the $X$ and $Y$ plane of piezoceramic wafer in order to act as a waveguide for ultrasonic waves and hence improve the imaging resolution and performance over the bulk material [12]. The cuts are filled with an interstitial material such as an epoxy or polymer.

during the casting process. In the subsequent ceramic firing process the fibers can be burned out, leaving a controlled density of aligned pores. The presence of these pores controls the expansion and contraction of the material, effectively acting as a waveguide to ultrasonic waves.

The waveguiding effect is dependent on the degree of alignment and spatial density of the pores, thus essential to be able to control these parameters during the casting process. Transmission optical microscopy is commonly used for observing fiber alignment [13] [14], however piezoceramic wafers are created using lead zirconate titanate (PZT), an opaque material. This makes in situ optical monitoring and characterisation of the fiber motion and hence the resulting piezoceramic wafer unachievable.

This Masters project forms part of the Callaghan Innovation research and development work for the production of such piezoceramics with aligned pores. The focus of the work is as follows:

- Research existing methods for optical and electrical monitoring of parti- 
cles and fibers in fluid suspensions.

- Develop and implement techniques for the optical and electrical monitoring of magnetic fiber rotation when subjected to a linear magnetic field in a transparent fluid.

- Develop and implement an optical and electrical technique for monitoring the magnetically assisted sedimentation of magnetic fibers.

- Compare the results of optical and electrical techniques to analytical models for fiber rotation and translation.

The structure of the thesis is as follows: Background theory introducing magnetics and the magnetic properties of materials, including the manipulation and monitoring of magnetic particles is presented in Chapter 2. The experimental equipment and procedures developed for this project are explained in Chapter 3. Theoretical modeling and experimental results of fiber rotation and translation are presented in Chapters 4 and 5 respectively. Finally, Chapter 6 provides discussion of the key results, conclusions, and future work. 


\section{Chapter 2}

\section{Background}

This chapter introduces the basic theory of magnetism and the magnetic properties of materials. Techniques for manipulating, tracking, and measuring magnetic particles are then discussed. Finally, literature regarding these techniques is analysed.

\subsection{Magnetism}

Magnetic flux will permeate through a vacuum as a result of an applied field $\vec{H}$. At a given point in space, the magnetic flux density $\vec{B}$ is given by

$$
\vec{B}=\mu_{0} \vec{H}
$$

where $\mu_{0}$ is the permeability of free space, with a defined value of $4 \pi \times 10^{-7}$ $\mathrm{H} / \mathrm{m}$. In this case, the magnetic flux density is dependent solely on the applied field. A material introduced to this space interacts with the applied field, and affects the magnetic flux distribution. The magnetic flux density becomes

$$
\vec{B}=\mu_{0}(\vec{M}+\vec{H})
$$

where $\vec{M}$ is the magnetic moment per unit volume commonly referred to as the magnetisation. The magnetisation $\vec{M}$ resulting from an applied field $\vec{H}$ may be represented by

$$
\vec{M}=\chi \vec{H}
$$


where $\chi$ is the volume magnetic susceptibility of the material. This value for $\chi$ describes how the material interacts with the magnetic field. For materials with $\chi$ greater than 0 , the material is said to be "paramagnetic"; the magnetisation is higher than that of free space. Conversely, materials with $\chi$ less than 0 are said to be "diamagnetic"; the material tends to exclude a magnetic field from its interior.

For other forms of magnetism $\chi$ is a tensor, meaning its value will be dependent on both the current strength of the applied field and any previous field exposure.

\subsubsection{Diamagnetism}

Diamagnetism is an inherent property of all matter due to the subatomic structure of individual atoms. Atoms consist of bands of electrons orbiting a proton-neutron nucleus. The proton and neutron in turn are comprised of quarks [15] [16]. Quarks and electrons are fundamental particles known as fermions which have spin magnetic moments resulting in a bulk material's magnetic properties. Fermions have a spin of $1 / 2$ in either the up or down direction, denoted as spin-up or spin-down. Fermions obey the Pauli exclusion principle where there can not be two fermions having the same position, velocity and spin direction [17]. As a result, spin-up and spin-down electrons form pairs in the orbitals of atoms. These opposing spin directions effectively cancel out the spin magnetic moments of the electrons, leaving the magnetic properties as a function of only the spin magnetic moments of the nucleus [18]. These materials are repelled by a magnetic field however the effect is miniscule and common diamagnetic materials are thought of to be non-magnetic. These include water, organic compounds, and gold [19]. The repulsive force acting on highly diamagnetic materials, including superconductors and pyrolytic carbon, has been demonstrated to result in the levitation of the material above a strong magnetic field, illustrated in Figure 2.1. All materials exhibit diamagnetism, but as the effect is very small $\left(\chi \approx-10^{-5}\right)$, diamagnetism is masked by all other forms of magnetism the material may exhibit. 


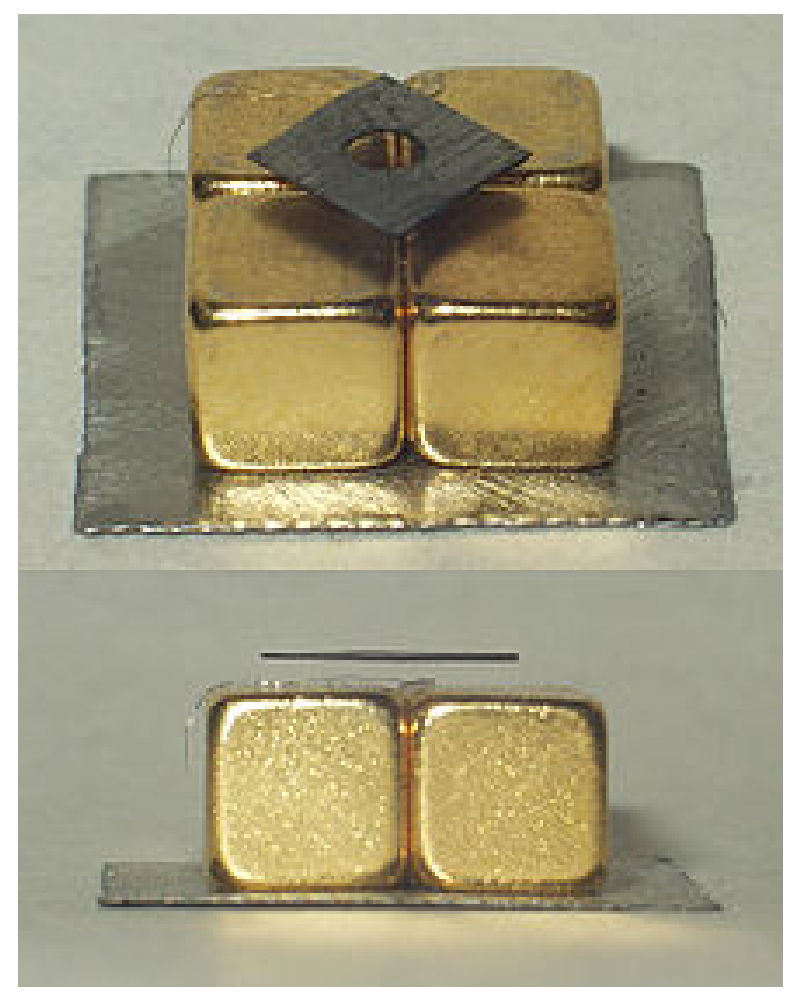

Figure 2.1: Materials with a large diamagnetic constant levitate above strong magnets due to the repulsion force. Pyrolytic carbon is a material with one of the highest diamagnetic constants at room temperature. The magnets illustrated here are $1 \mathrm{~cm}$ cubes. 


\subsubsection{Paramagnetism}

Materials with unpaired electrons and hence incomplete orbitals are paramagnetic. These unpaired electrons create a magnetic dipole moment in the material [20]. Due to thermal agitation these dipole moments are randomly distributed throughout the material giving the material a net zero magentisation. The application of an external magnetic field results in the spin direction aligning parallel to the external field, giving the material a non-zero magnetic moment [21]. This magnetic moment is dominated by aligned dipole moments, however there is still a small diamagnetic component. The temperature dependance of many paramagnetic materials can be explained by the Curie-Weiss law [20],

$$
\chi=\frac{\mathrm{C}}{\mathrm{T}-\theta}
$$

which states the magnetic susceptibility, $\chi$, is inversely proportional to the absolute temperature, $\mathrm{T}$, adjusted with a constant, $\theta$, which has the same units as the temperature and may have positive or negative values. In general, metals do not obey the Curie-Weiss law.

Conductive materials such as metals exhibit Pauli paramagetism due to the electron locatisation in the material [22]. In conductive materials, the electrons can be considered as free electrons (delocalised) able to move through the bulk of the material. The conduction band for such a material is identical for both spin-up and spin-down electrons, however with the application of a magnetic field the conduction band splits apart into a spin-up band and a spin down band due to the difference in magnetic potential energy. As the Fermi level must be identical for both bands, there will be a small surplus of the spin direction with less energy [23]. This effect is stronger in $\mathrm{d}$ - and $\mathrm{f}$ - electrons compared to s- and p- electrons due to a larger sensitivity to conduction band shifts associated with a stronger electron localisation [24]. However in all cases Pauli paramagnetism is weaker than regular paramagnetism due to only the electrons near the Fermi level contributing to the materials magnetisation.

The magnetisation of Paramagnetic and Pauli paramagnetic materials increases linearly with the applied field strength until a saturation magnetisation 


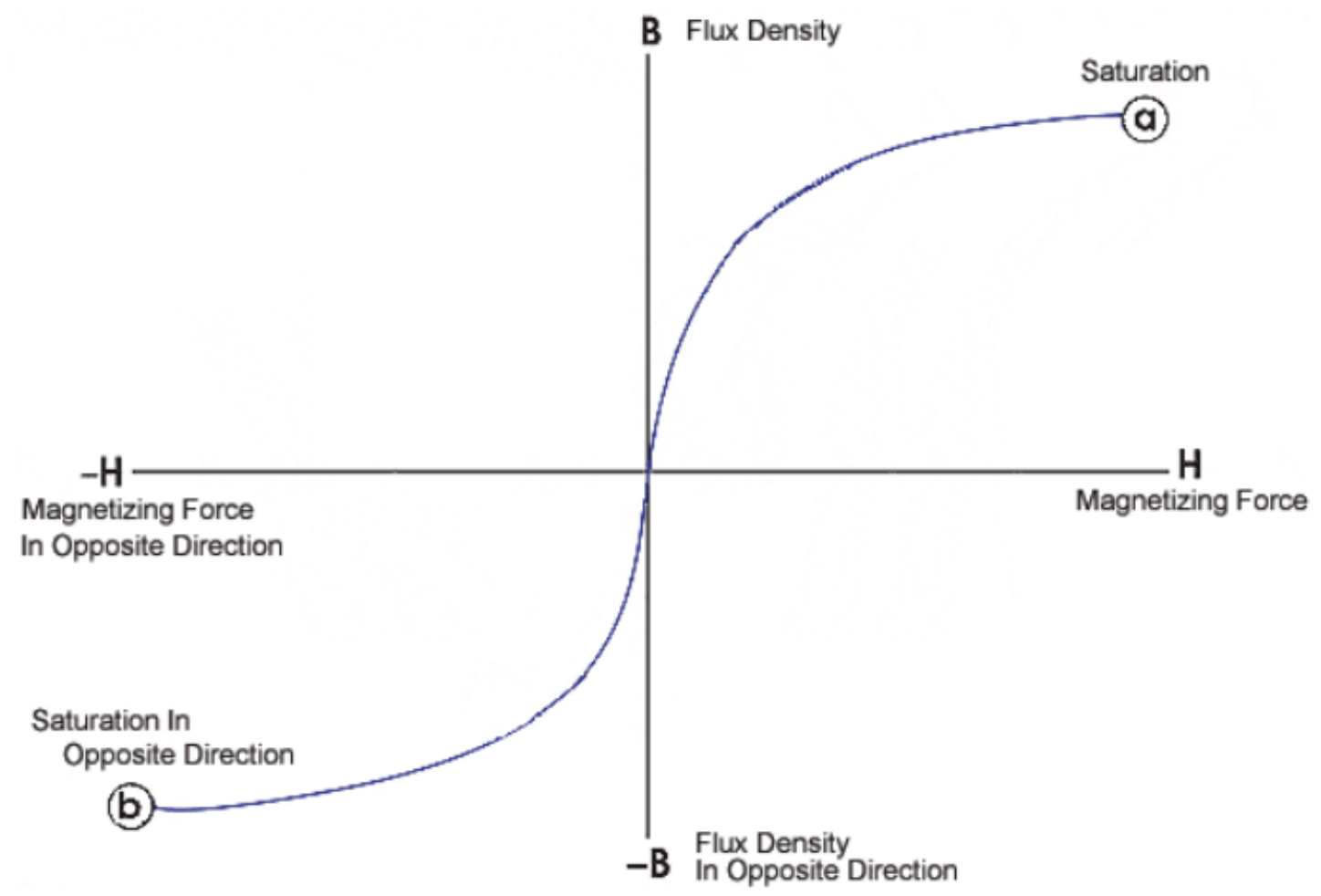

Figure 2.2: The general magnetisation curve for paramagnetic materials has no remnant magnetisation when the magnetic field is removed. The magnetic flux density saturates both positively (a) and negatively (b).

is reached, whereby an increase in applied field has no effect. The removal of the magnetic field results in the material returning to its initial demagnetised state with dipole moments randomly oriented and a zero net magnetic moment. These effects are illustrated in Figure 2.2, where a general magnetisation curve for paramagnetic materials is presented.

\subsubsection{Ferromagentism}

Ferromagnetic materials exhibit the magnetic dipole phenomena observed in paramagnetic materials, however they retain their magnetisation with the removal of a magnetic field [25]. In ferromagnetic materials, electrons which repel one another reduce the electrostatic energy, and hence tend to become more sta- 
ble when the spins of unpaired electrons are parallel compared to anti-parallel. This is called the exchange energy; the energy between parallel and anti-parallel spin direction.

This exchange keeps the dipole moments with a specific domain aligned, but not in any particular direction. The magnetic anisotropy of the material keeps the spin orientation stable, without it the orientation would change randomly with thermal fluctuations.

In a demagnetised state, ferromagnetic materials have a number of domains, each with aligned spins in a random orientation illustrated in Figure 2.3 [26]. This is due to the decay of the exchange interaction over distance, hence over long distances the spins align in the opposite direction due to the dipole energy overcoming the exchange interaction. At the border of these domains, the spin direction rotates between the two competing domains direction. These domains can be altered with the application of an external magnetic field until the material is in magnetic saturation, where all domains are aligned and an increase in magnetic field strength has no effect. These domains become fixed with the removal of the magnetic field due to the domain walls 'snagging' on defects in the crystal lattice, preserving their parallel orientation.

The magnetisation as a function of external field strength for a ferromagnetic material can be described by the hysteresis curve illustrated in Figure 2.4. This describes the magnetisation saturation of the material, as well as both the retentivity; the magnetisation remaining after the removal of the applied field, and the coercivity; the magnetic field strength applied in the opposite direction required to demagnetise the material.

Superparamagnetism [27] is a form of magnetism exhibited by small ferromagnetic particles which contain a single domain, typically with a diameter of $3-50 \mathrm{~nm}$. Single domain particles have a hysteresis curve with no retentivity or coercivity. Due to the materials magnetic anisotropy, the magnetic moment of superparamagnetic particles typically have only two stable orientations antiparellel to each other, separated by an energy barrier. Due to thermal fluctuations the magnetic moment can flip between these two directions. 


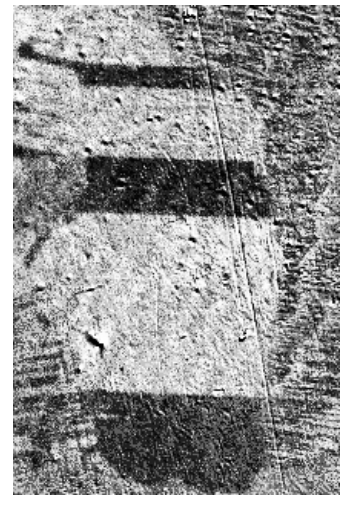

(a)

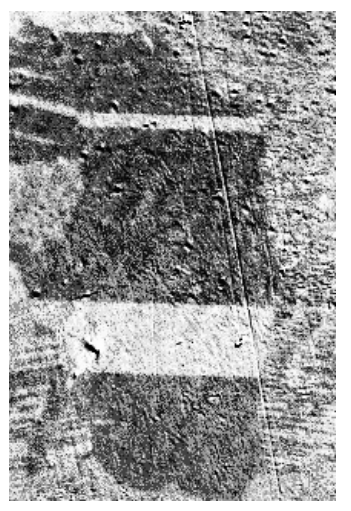

(d)

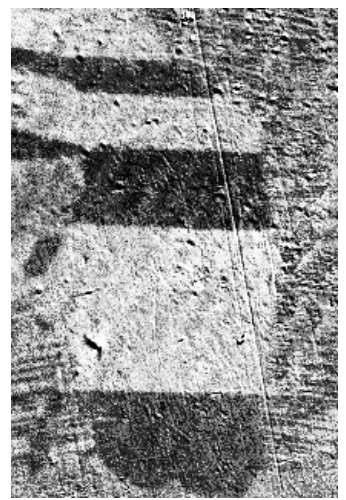

(b)

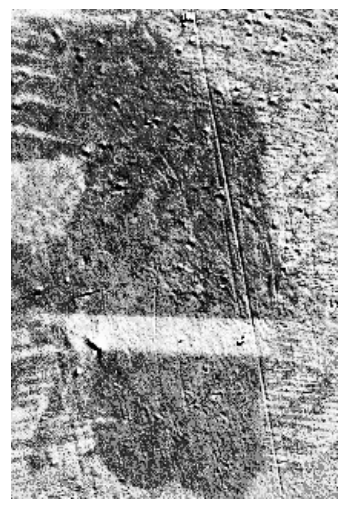

(e)

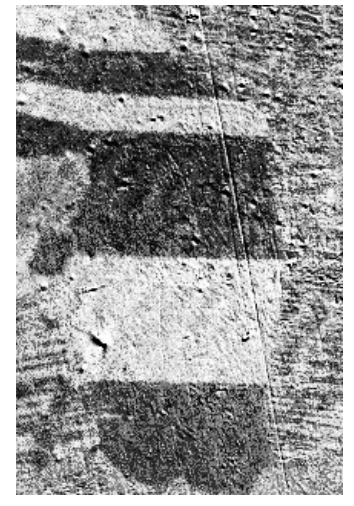

(c)

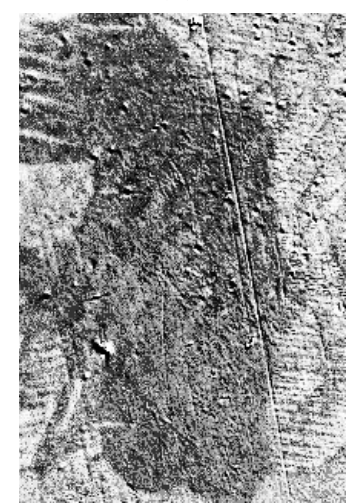

(f)

Figure 2.3: Moving domain walls in a ferromagnetic material due to an applied external field, observed with a Kerr-effect microscope. The sample starts in a demagnetised state (a), applying an increasing magnetic field in a downward direction (b-e), and finally saturating (f). Light areas have magnetisation upwards and dark areas have magnetisation downwards. 


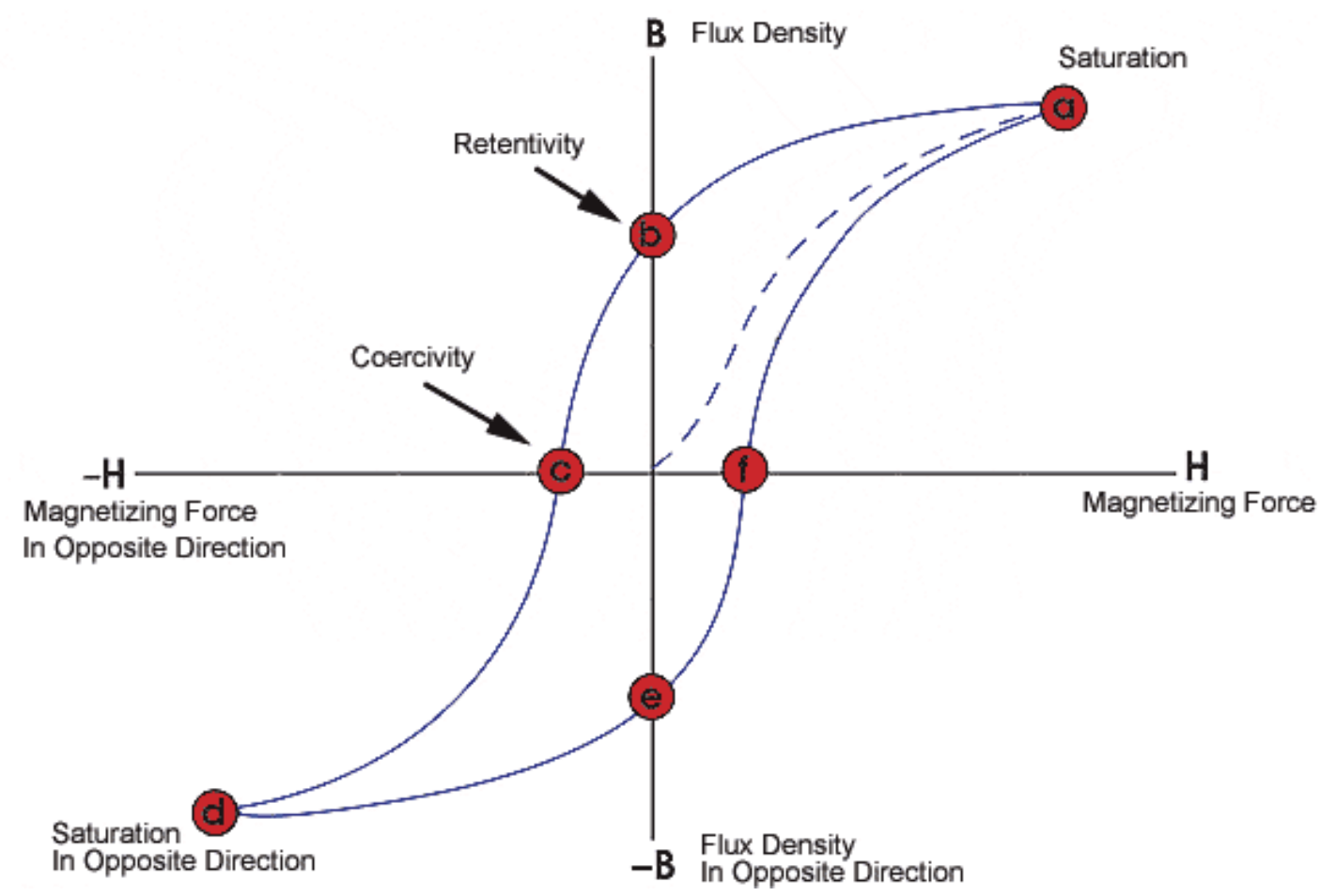

Figure 2.4: An unmagnetised ferromagnetic material exposed to an external magnetic field becomes magnetised following the dashed line until it becomes magnetically saturated (a). The removal of the magnetic field results in remnant magnetisation (b). The application of a increasingly negative magnetic field demagnetises the material (c), until the material reaches magnetic saturation in the opposite direction $(\mathrm{d})$. Removing the negative magnetic field results in remnant magnetisation (e), and the application of a positive magnetic field demagnetises the material (f). Further increasing the positive magnetic field results in positive magnetic saturation (a). 


\subsection{Synthesis and Implenetation of Magnetic Parti- cles}

Magnetic particles have a wide variety of uses including environmental [28] and medical applications [29], and creating anisotropic properties in composites [30]. This section details the synthesis and implementation of magnetic particles across a range of applications.

Magnetic micro- and nanoparticles have been synthesised using magnetic materials imbedded in polymer microspheres. These particles can be tailored to bind to certain metal species or molecules when put in an aqueous solution, and passed through a High Magnetic Gradient Separation (HGMS) system where the magnetic particles collect in high field gradient regions. This separation has implications in the reprocessing of waste, for example the remediation of heavy metals, and for the isolation of contaminants from water [28].

Magnetic particles can also be synthesised with ligands bound to the polymer matrix. This allows for the particles to be bound to a diverse rage of organic substances such as cells, proteins, and nucleic acids. Anion-exchange magnetic particles carrying diethylaminoethyl groups were synthesised to ionicly bind pharmacological or biological substances or drugs. These modifications to the particles allow for the use in biomedical applications such as targeted drug delivery and clinical analysis of biological samples. Magnetic particles used in this manner have benefits over chemotherapy and radiotherapy due to the nonspecificity of these treatments even under ideal conditions, resulting in damage to otherwise healthy tissues.

Magnetic fluid hyperthermia is the treatment of cancer cells using heat. This process relies on the manipulation of a magnetic fluid to a target location and the subsequent heating of the fluid. Heat is generated in the magnetic fluid due to the magnetic hysteresis loss when subject to a variable magnetic field [31]. Cancer cells are destroyed at temperatures of $42-46{ }^{\circ} \mathrm{C}$ whereas normal cells can survive at these temperatures, making this technique an effective targeted treatment for cancerous cells. The magnetic fluids used in this procedure utilise 
the superparamagnetic properties of iron oxide nanoparticles [29].

The addition of magnetic microwires to a composite material gives it anisotropic or high frequency responce properties. The microwire composition varies from application to application. For example, nickel coated carbon fibers added to a conductive composite can be used for electromagnetic interference shielding. This high frequency shielding is important especially with the demand for reliable electronics and the rapid growth of radio frequency radiation sources [32]. Similar EMI shielding can be achieved using carbon coated iron nanocapsules [33].

Two dimensional nanocomposites fabricated using a host matrix and magnetically aligned nanoscale-diameter wires [34]. Potential applications include thermal insolation in microelectronics [30], thermoelectrics, boiling surfaces in cryogenic applications [35], and low-dielectric constant dielectrics [36]. The efficiency and energy density of thermoelectric devices are determined by the figure of merit

$$
\mathrm{ZT}=\frac{S^{2} \sigma T}{k}
$$

where $S$ is the Seebeck coefficient, $\sigma$ is the electrical conductivity, $k$ is the thermal conductivity, and $T$ is the absolute temperature. Phonons in nanostructured materials has shown to increase ZT compared to their bulk counterparts [37] [38] [39]. The thermal conductivity of such composites is dependent on the volume fraction and diameter of the imbedded nanoscale wires, and hence can be tailored for a number of practical applications.

The performance and properties of composites can be tailored for specific applications through the addition of nano- or microfibers. An increase in the elastic modulus of polymer nanocomposites has been observed with the addition of either randomly oriented or aligned multi-walled carbon nanotubes (MWNT) [40]. The load transfer between aligned carbon fibers and a polymer matrix has been demonstrated to significantly improve the ultimate strength and yield of polymer composites reinforced with aligned carbon fibers [40]. Anisotropy in the magnetic susceptibility, electrical conductivity, and elastic modulus of a polyester resin containing magnetically aligned MWNTs have 
been demonstrated [41].

\subsubsection{Manipulation of Magnetic Particles}

Magnetic particle manipulation refers to the manipulation of magnetic materials in $3 \mathrm{D}$ space, including position and orientation control, which is commonly achieved through the manipulation of magnetic flux through a material.

The combination of a constant magnetic field with magnetic particles allows for the alignment of magnetic particles parallel with the flux lines from a magnet. This technique has been demonstrated to align superparamagnetic material coated graphite flakes during the electrode casting and drying processes in the fabrication of electrodes for high-rate performance lithium ion batteries [42], illustrated in Figure 2.5. This alignment resulted in an improvement in the transportation diffusion mechanisms in highly loaded electrodes through creating a shorter diffusion pathway for lithium ions across the anode.

The introduction of a field gradient allows for the attraction of magnetic particles to the surface of the magnet creating a small region of densely populated particles. This technique, illustrated in Figure 2.6, has shown successful manipulation of intravenously injected pharmaceutical drugs bonded to a magnetic fluid. An externally located permanent magnet has been used to attract the magnetic chemicals to a target location, effectively trapping the drug. A number of permanent magnets were used to create unique magnetic fields to match the shape of tumors, with successful treatment of unsuccessfully pretreated cancer and sarcomas demonstrated using this method [43]. This magnetic guidance technique has shown promise for the development of targeted treatments, with research focusing on the development of magnetic drugs to use in conjunction with this procedure [44] [45] [46].

This technique is also used in microfluidic systems where magnetic particles are bonded to a material. The force exerted on a particle is a function of its magnetic moment, hence when the magnetic particle binds to a substance, a series of parallel channels can be used in conjunction with a permanent magnet to separate the particle from the bulk fluid [47]. 


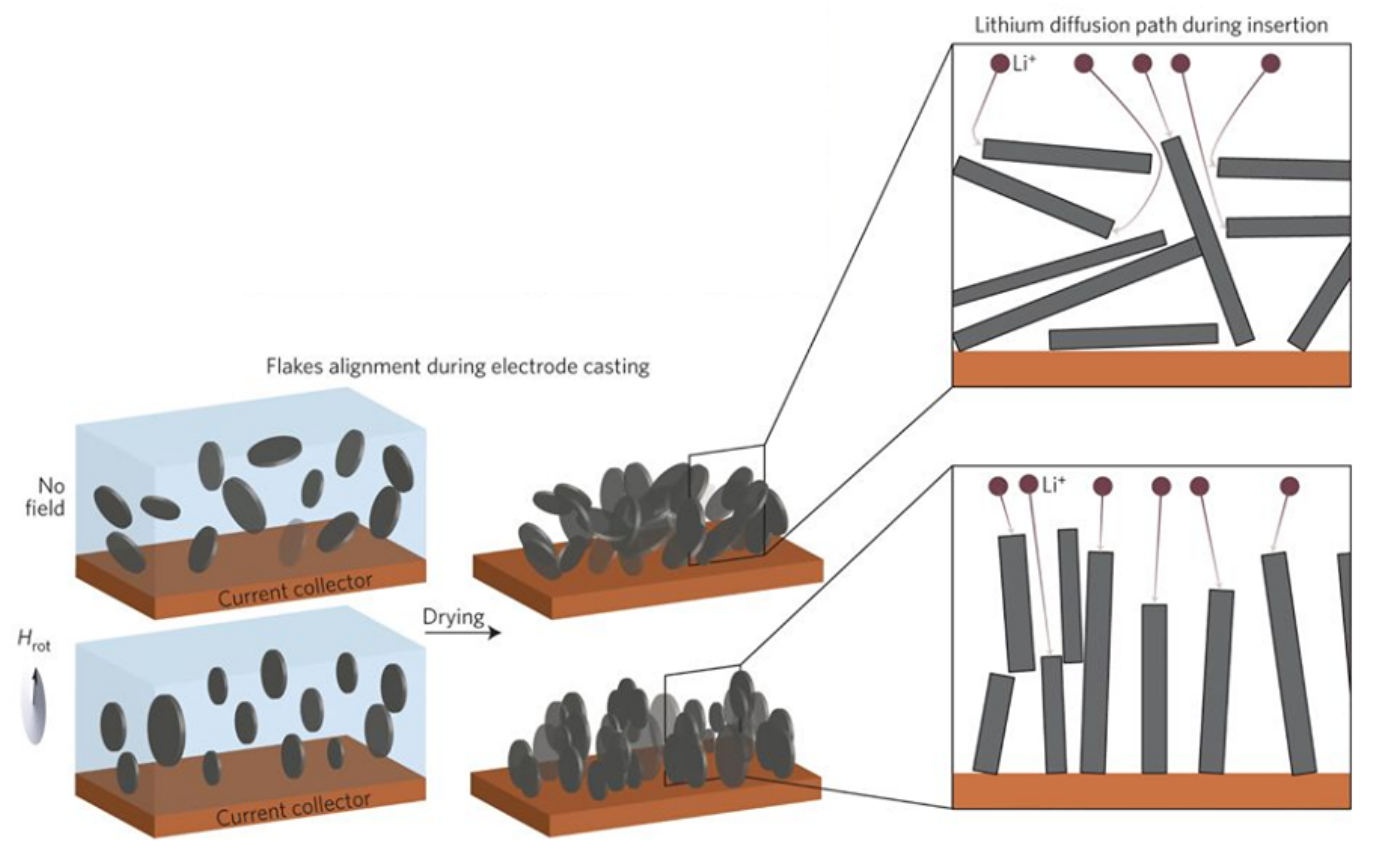

Figure 2.5: Graphite flakes coated with a superparamagnetic material can be aligned using a magnetic field during the casting and drying process [42]. 


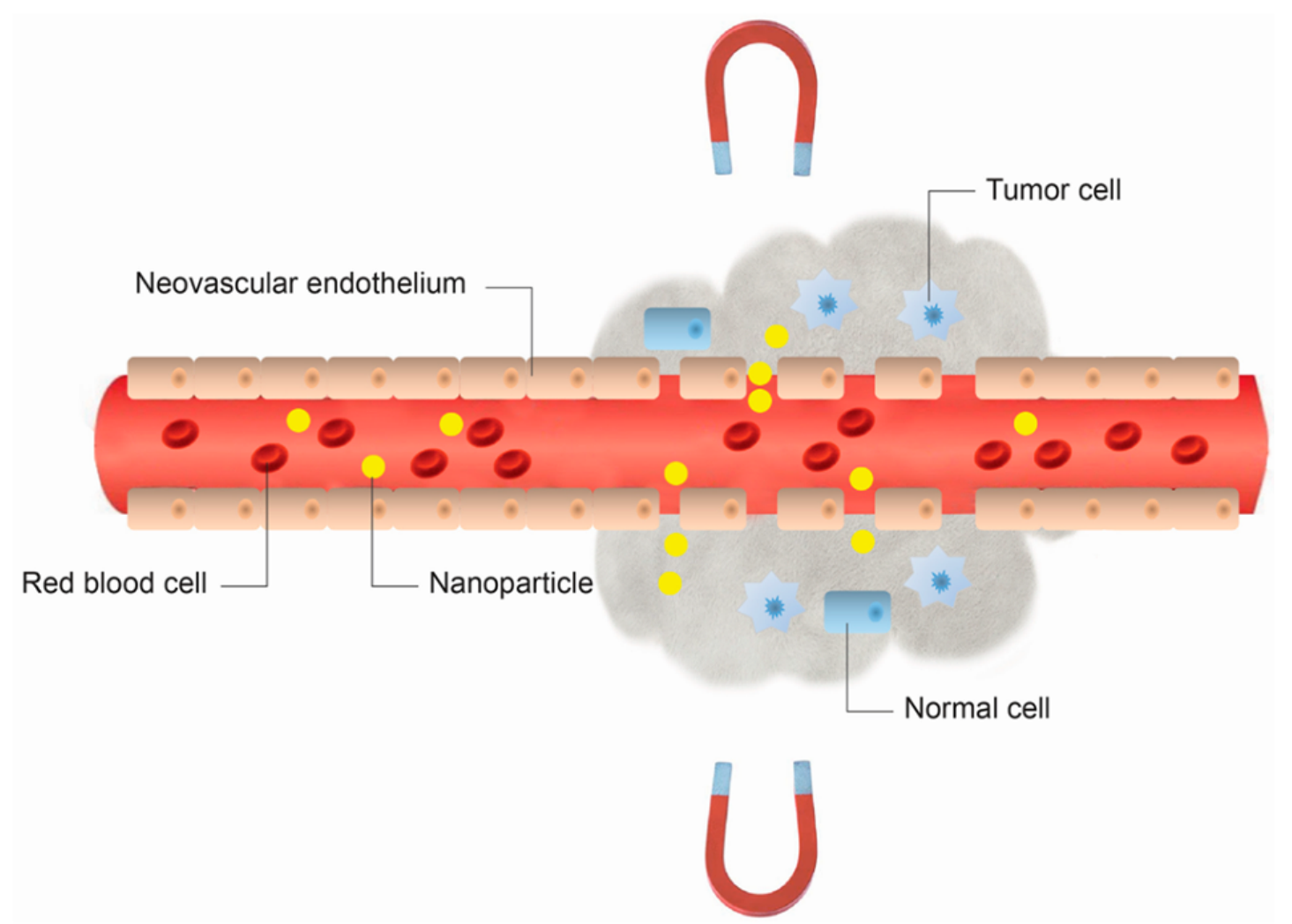

Figure 2.6: Pharmaceutical drugs bonded to magnetic nanoparticles can be manipulated to a target location for tumor treatment using external permanent magnets. 
The magnetic field can be manipulated using electromagnets as opposed to permanent magnets. The combination of three electromagnets orthogonal to one another allow for both position and orientation manipulation of a magnetic material in 3D space. Traditionally, catheters are operated manually through the rotation of the guide wire to the desired path, and then manual advancement or retraction of the sleeve until the target destination is reached. However, the modification of the catheter to have a magnetic tip in conjunction with orthogonal electromagnets removes the need for the rotation step, simplifying catheter based procedures. This technique has been demonstrated to provide fine control of these magnetic catheters for use in electrophysiology studies ${ }^{1}$ and cardiac ablation of arrhythmias ${ }^{2}$ in humans [50].

\subsubsection{In Situ Monitoring of Magnetic Particles}

Magnetic particle imaging (MPI) is a tomographic ${ }^{3}$ imaging method which directly measures the magnetisation of superparamagnetic iron oxide nanoparticle (SPIONs) tracers [51]. The signal generated by the SPIONs as a response to an oscillating magnetic field produces high order harmonics of the excitation frequency [52] [53] [54], allowing high spatial and temporal resolution mapping of the local SPIONs [55] [56]. As biological organs are diamagnetic they appear transparent in MPI measurements eliminating issues with background signals. The basic components of an MPI system are illustrated in Figure 2.7 whereby the oscillating magnetic field is generated by the application of a current through transmit coils. An additional static magnetic field is superimposed onto the drive field to create a region of space with a net zero magnetic field containing the SPIONs of interest. The resulting magnetic flux density is evaluated through measuring the induced voltage on the receive coils [51].

A single sided MPI device capable of 1D imaging has been created using two

\footnotetext{
${ }^{1}$ Electrophysiology is the study of ion current in biological tissues [48].

${ }^{2}$ Cardiac ablation is a procedure to correct an abnormal heart rhythm through the scarring or destruction of tissue in the heart [49].

${ }^{3}$ Tomography is imaging by sections using any kind of penetrating wave.
} 


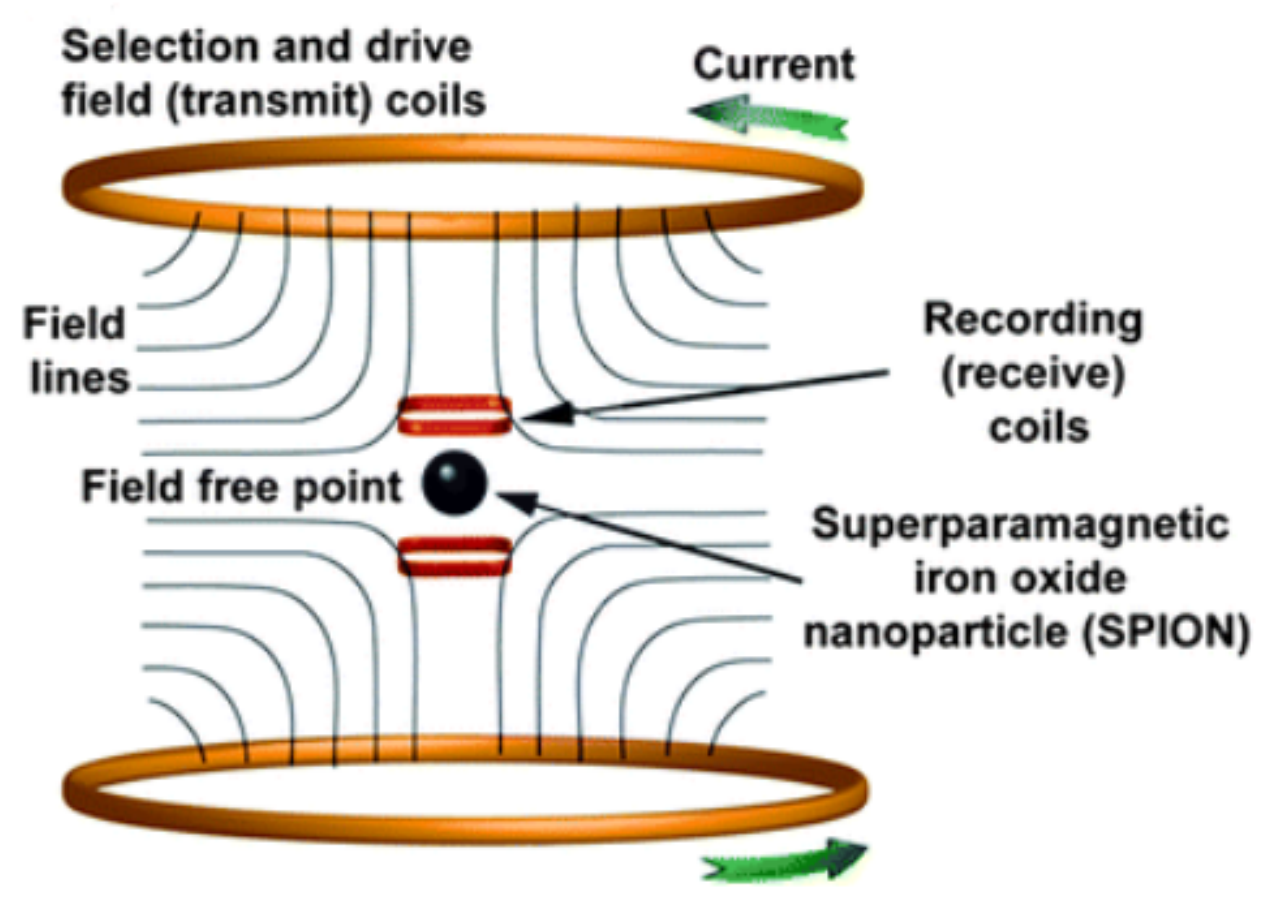

Figure 2.7: Schematic representation of an MPI system used for measuring superparamagnetic iron oxide nanoparticle (SPIONs) in organic material. A magnetic field is generated using transmit coils, with the resulting flux density around the SPIONs measured as a voltage induced on the receive coils indicating the position and density of SPION particles [51]. 
concentrically positioned transmit coils and a single receive coil sandwiched between them [57]. Regular MPI devices are required to enclose the sample, however this device allows for a sample to be placed above the device, and hence removes the size limitations traditional devices suffer from. The image resolution is a function of field strength, which decreases as a function of distance. This makes these single sided MPIs applicable for near surface imaging with high resolution up to a depth of several centimeters [57].

Whist magnetically assisted delivery of magnetic nanoparticles have been implemented in targeted drug delivery, drugs delivered to healthy tissue can cause undesirable side effects. Real time monitoring of magnetic nanoparticles can improve the efficiency of such a targeted drug delivery system. One method for two axis magnetic particle manipulation with simultaneous location measurement requires four actuation coils to generate a field gradient illustrated in Figure 2.8 [58]. A cancellation and a receive coil is then placed inside an excitation coil orthogonal to the 4 actuation coils. 


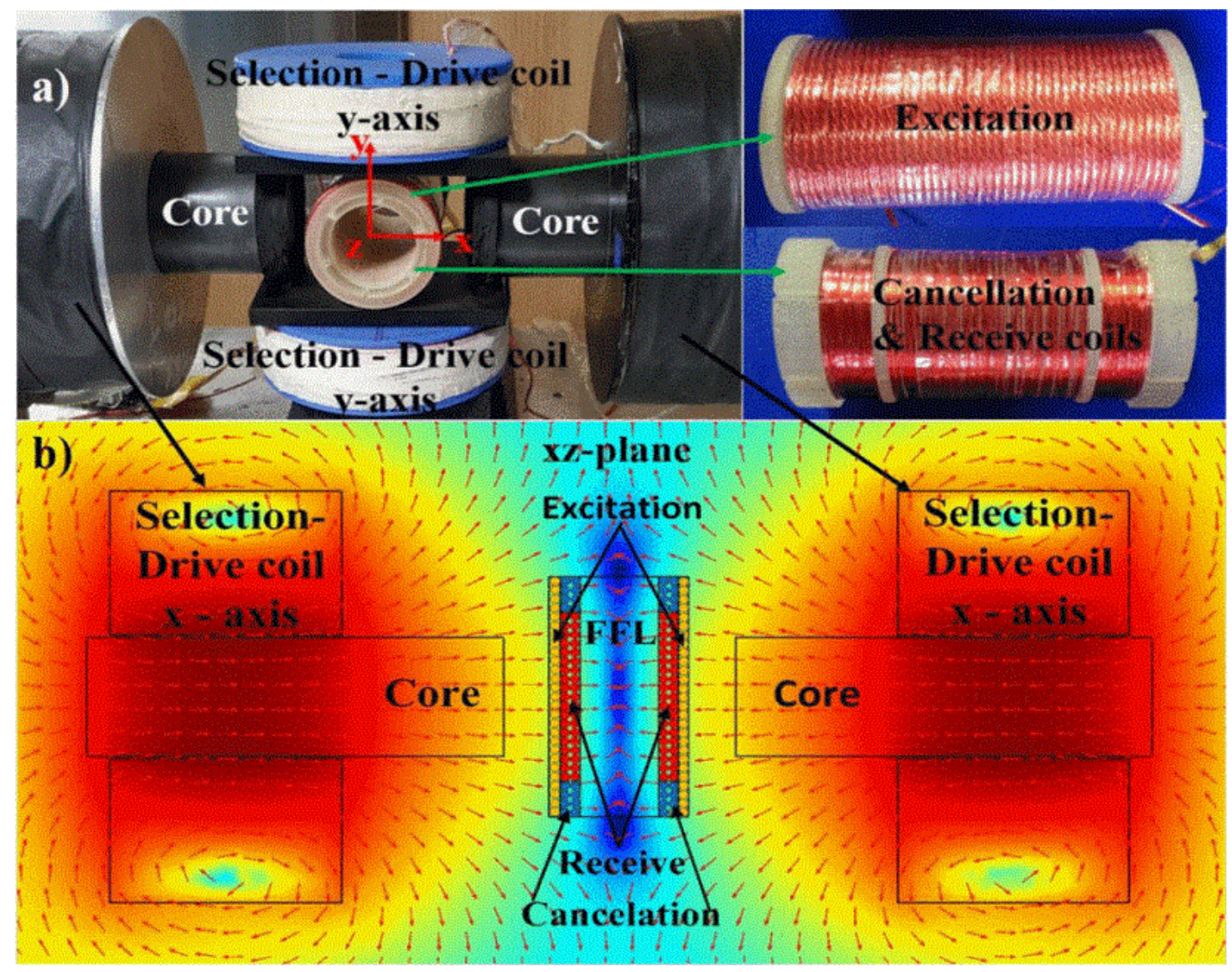

Figure 2.8: The experimental design of the 2D magnetic particle manipulation system (a) consists of selection/drive coils orthogonal to an excitation coil enclosing a receive coil. The resulting field profile inside the system controls the magnetic particles position over time. 


\subsection{Fabrication of Porous Ceramics}

This section provides a brief overview of the process for the fabrication of porous ceramics under development at Callaghan Innovation, and the forces a fiber will experience during this process.

The creation of porous ceramics involves creating a carbon fiber-filled PZT ceramic slurry and inserting it into a mold. The mold is placed into a magnetic field which aligns the fibers, and attracts them to the bottom of the mold. The ceramic is then hardened and sintered, burning out the fibers and leaving an array of aligned pores. Figure 2.9 illustrates this process in 6 steps. The degree of alignment in the bottom of the mold is dependent on the angle of the flux lines from the magnet relative to the mold, so a metal cell containing a permanent magnet was created which provides a high field gradient for attracting fibers and orients the flux lines perpendicular to the magnets face.

A ceramic slurry is comprised of the PZT powder, an organic binder, a dispersant, and water. The ratio of these materials defines the resulting ceramics properties. The ceramic slurry formula for the fabrication of porous ceramics is under development, with initial compositions producing fragile wafers which are prone to cracking. From the perspective of this thesis, this makes the development of a specific method for measuring the fiber motion challenging as the specific properties of the final ceramic slurry are undefined, hence the method should be suitable for fibers suspended in a general fluid.

\subsubsection{Forces of a Fiber During Ceramic Fabrication}

The forces acting on a fiber during the fabrication of porous ceramics will dictate the fiber dynamics and hence the quality of the final wafer. These forces are briefly discussed as follows:

\section{Gravitational force}

The gravitational force acting on a fiber results in the natural sedimentation of the fibers. The sedimentation effect in combination with drag aligns slender 


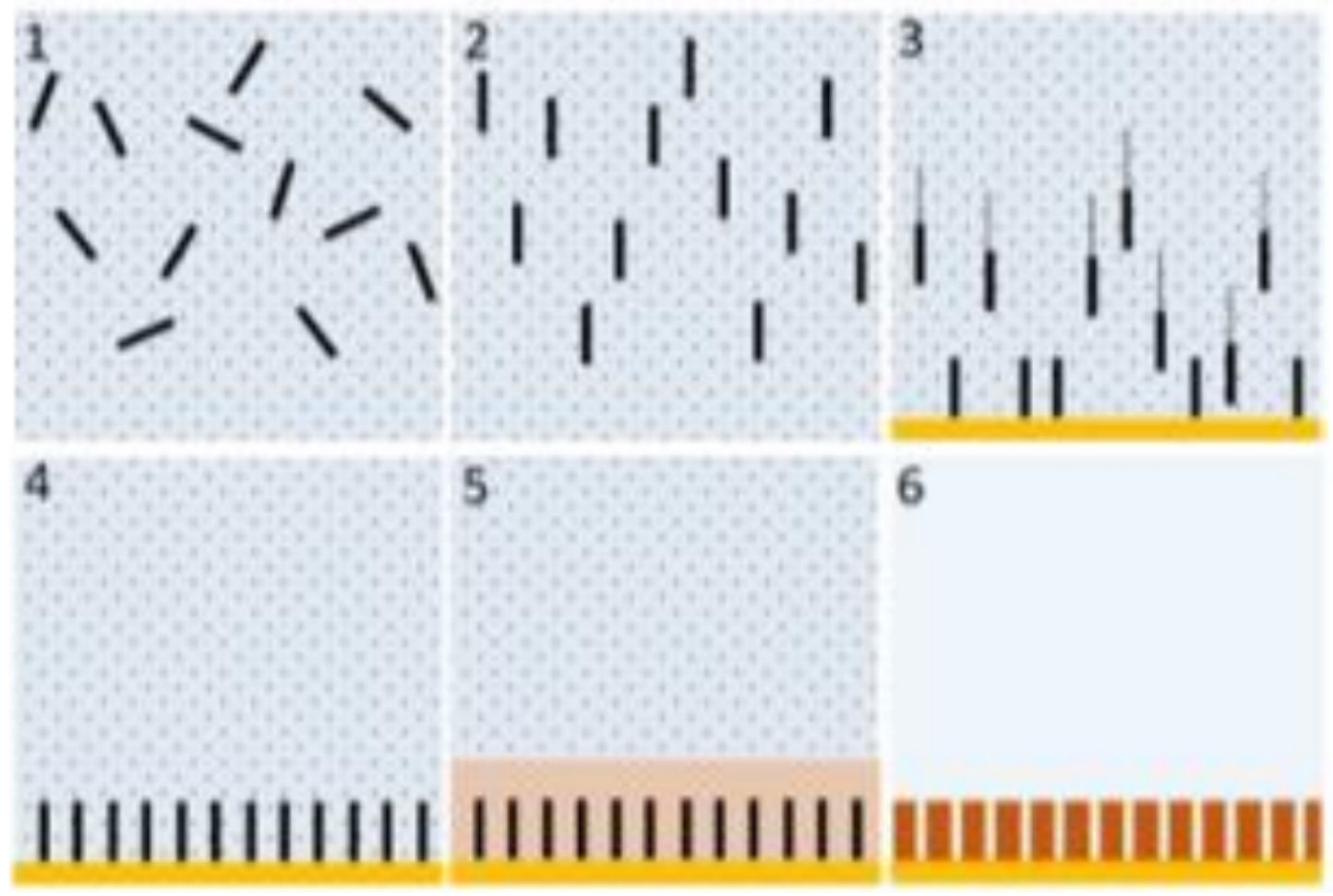

Figure 2.9: The process under development for the fabrication of porous ceramics under development at Callaghan Innovation can be simplified into 6 steps. Firstly, a PZT ceramic slurry containing fibers is created and inserted into a mold (1). The fibers are then aligned via a magnetic field (2), and attracted to the surface of a permanent magnet (3). The fiber form an array at the bottom of the mold (4) add the ceramic is hardened (5). Finally, the ceramic is sintered, burning out the fibers and leaving an array of aligned pores. 
bodies parallel to the bottom of the ceramic mold [59], which is the opposite of what is required for the fabrication of aligned pores. This force acts on all particles in a fluid, hence it should be included in the analysis of fiber motion.

\section{Hydrodynamic Drag}

The hydrodynamic drag force opposes the motion of an object [60]. This force in combination with gravitational forces aligns a slender body parallel to the bottom of the ceramic mold. The drag force is proportional to the objects velocity, which imposes a terminal velocity to an object in free fall, hence it is important to determine the influence this force has on fiber motion on the time taken for a fiber to reach the bottom of the mold.

\section{Buoyancy Force}

Buoyancy is the upward force which opposes the weight of an immersed object [61]. The magnitude of this force is dependent on the mass of the displaced fluid, so for the ceramics applications this imposes lower limits on the magnetic field gradient generated in the magnetic cell. This is because the PZT is a dense material $\left(7600 \mathrm{~kg} / \mathrm{m}^{3}\right)$ when compared to carbon $\left(2267 \mathrm{~kg} / \mathrm{m}^{3}\right)$. In order to mitigate this buoyancy force, either the PZT component of the slurry should be kept to a minimum or the nickel coating on the fibers should be large as nickel has a density of $8909 \mathrm{~kg} / \mathrm{m}^{3}$. In any case, analysis of this force is crucial in the successful creation of porous ceramics.

\section{Magnetic Attraction}

The magnetic attraction force is used to overcome buoyancy forces during the fabrication of porous ceramics. This force is proportional to the magnetic field gradient [62], which is maximised through the magnetic cell. Fibers subjected to a weak magnetic field gradient may either float to the top of the mold, or end in a stagnation point where the magnetic force may not overcome the the other forces, resulting in a net force of zero. Typically this point would be at a 
region where the magnetic fluid gradient changes from positive to negative or vice versa.

\section{Hydrodynamic Torque}

The hydrodynamic torque [63] on a fiber in a ceramic slurry is dependent on the motion of the fluid. In laminar flow, fibers tend to align with the direction of the flow, whereas turbulent flow fiber orientation and velocity will be be essentially random. Hydrodynamic torque is particularly important during fiber rotation, where it opposes the alignment of fibers with the magnetic field.

\section{Magnetic Torque}

The magnetic torque is a product of the magnetic field strength, and results in the alignment of the fibers with the field lines [63]. This force must overcome the horizontal fiber alignment due to sedimentation effects, and the hydrodynamic forces opposing the change in fiber orientation.

\section{Brownian Motion}

Brownian motion [64] is the motion of a particle suspended in a fluid due to its collision with fast moving molecules in the fluid. The motion of the molecules is random in all directions, so for large particles in the fluid the Brownian motion has a net zero effect due to collisions on all sides of the particle. Particles greater than $100 \mathrm{~nm}$ do not exhibit Brownian motion, hence the force from these collisions can be ignored.

\section{Stress and Strain}

Fibers in motion in a fluid are subject to shear stress at the fiber-fluid boundary [65]. For Newtonian fluids (which we aim to use), the shear stress is linearly proportional to the strain rate, and is encompassed in the drag calculations as skin friction. 
The strain on a fiber due to external loads such as gravity, or a temperature change may lead to the deformation of the fiber should the load be high enough. This significant deformation of the fibers will alter the fiber dynamics. During the fabrication of porous ceramics, the fibers are not expected to experience significant external forces where deformation may occur, and high temperature changes where thermal expansion occurs will be during the sintering process when the fibers are to be burned out of the ceramic. This means both stress and strain effects can be considered to be negligible.

\subsection{Literature Review of Magnetic Alignment and Sedimentation of Slender Bodies}

The magnetic alignment of microwires has been reported in literature since 1972, where Timbrell demonstrated the alignment of high-modulus carbon fibers and iron coated man-made fibers in a liquid suspension via magnetic fields [66]. The effect of field strength and particle size on the alignment of rodlike and platelet-like particles in a host matrix was explored by Erb, where the ideal particle size for minimising the required applied magnetic field strength was determined to be in the range of $10 \mu \mathrm{m}$ [67]. The motion of particles smaller than this is dominated by thermal effects and larger particles are dominated by gravitational effects, hence both require higher magnetic torque to align them. However, the monitoring of these microwires and microparticles are strictly optical.

Michaud demonstrated the magnetic alignment of weakly diamagnetic ceramic fibers through transmission optical microscopy using a CCD camera. The camera was placed in a magnetic field beside a brightly backlit sample container [13]. The experiment used glycerin with a fluid viscosity of 1.49 Pas as a suspension medium to avoid sedimentation, while fiber rotation was recorded over a 90 degree range. In-situ transmission optical microscopy has was used by Takahashi to measure the alignment of vapor-grown carbon fibers (VGCFs) in polydimethylsiloxane [14] when subject to magnetic and electric fields. Struc- 


\subsection{LITERATURE REVIEW OF MAGNETIC ALIGNMENT AND} SEDIMENTATION OF SLENDER BODIES

tural observations were made using transmission optical microscopy along two axis, monitoring the growth of VGCFs in 1 Pas silicone oil. Electrophoresis ${ }^{4}$ was determined to be a key factor in the resulting structures' properties.

The combination of modeling and optical monitoring of a single fiber is also prevalent in literature. The rotation of a suspended ferromagnetic fiber in a magnetic field has been modeled and evaluated by Shine and Armstrong [68]. The model consists of balancing the hydrodynamic torque and the magnetic torque acting on the fiber. The agreement between the model and experimental data is reported to increase with higher aspect ratios. Kimura derived an expression for fiber rotation through balancing the hydrodynamic torque and magnetic torque for a prolate ellipsoid [69]. The derived model is in good agreement with experimental results for both $30 \mu \mathrm{m}$ diameter polyethylene fibers and a $10 \mu \mathrm{m}$ diameter carbon fibers with lengths of $1,2,3$, and $4 \mathrm{~mm}$. Suspension solutions were crated using a water-ethanol mixture where the fibers were neutrally buoyant, with viscosities of 2.57 and $1.06 \mathrm{mPas}$ respectively. In both Shine and Kimura's experiments either only a single fiber is considered, or the number of fibers were limited to avoid fiber aggregates. Hence it is unknown how well the model will predict the motion of spatially dense fiber concentrations where inter-fiber interactions either physically or through magnetic dipole interactions.

Ciambella examined the rotation of a number of short magnetic fibers in a fluid with time dependent viscosity [70]. A simple model was derived to model the rotation of nickel coated carbon fibers in polydimethylsiloxane (PDMS) during the curing phase, where the viscosity increases as the PDMS solution solidifies. The specimen was placed between the plates of an electromagnetic which was used to generate the magnetic field, and the fiber orientation was captured using an optical microscope. The derived model shows good agreement with the experimental observations, where the fiber orientation distribution is a function of the geometric properties of the fiber and the curing rate of the PDMS

\footnotetext{
${ }^{4}$ Electrophoresis is the motion of dispersed particles relative to a fluid subjected to a uniform magnetic field.
} 
solution.

The translational motion of slender bodies in a fluid is a complex and well studied field [71] [72] [73]. Slender body theory is a methodology which takes advantage of a high length-to-width ratio to obtain an approximation of the net effect on the body through considering stokes flow [74]. Batchelor modeled the fluid-mechanical interactions between aligned fibers in semi-dilute suspensions [75]. Based on this work, Evans incorporated slender body theory into Batchelors model and assumed sufficiently large particle size such that Brownian motion can be omitted from the model [76]. Most recently, Borker has developed an extension for the slender body theory in which the resistance to translation is modeled for slender triaxial ellipsoids under stokes flow [77]. In resistive force theory, forces exerted on the slender deformable body by the surrounding fluid are described as externally applied normal and tangential tractions with velocity proportional magnitudes [78]. This theory is generally applied to flowinduced viscous forces on submerged bodies at low Reynolds number flows, and to the movement of microscopic organisms swimming in fluids [79]. These methods for modeling the motion of slender bodies are typically applied to either slender bodies subject to flow, as this is use in the fabrication of fiber reinforced composites [80] [81]. Ferec has modeled fiber interactions under flow using a hydrodynamic friction coefficient proportional to the relative velocity at the contact point [82]. The model includes a probability component dependent on the concentration of fibers per unit area. Similarly, Folgar has demonstrated their derived model can model the alignment of fibers under shear flow [83]. It is noted the fibers reach a steady state orientation, and the time taken to reach this state increases with fiber concentration and fiber length.

The motion of cylinders in free-fall is reported extensively in literature. Abelev simulated and dropped a $2.4 \mathrm{~m}$ cylinder with an aspect ratio of 4.5 to analyse the effects of changing the center of mass and the shape of the cylinder ends on the settling motion in water [84]. The effects of changing the center of mass from the center of volume resulted in a less stable orientation during free-fall. Furthermore, the even weighted cylinder demonstrated more lateral 


\subsection{LITERATURE REVIEW OF MAGNETIC ALIGNMENT AND SEDIMENTATION OF SLENDER BODIES}

motion as a result of hydrodynamic stability. Bragg modeled the unsteady rotation of cylinders in free-fall [85]. The model derived is determined to accurately describe the angular motion of the cylinder for low Reynolds numbers near Stokes flow. It is also noted surface roughness asymmetries and deformations may alter the free fall behavior. Chhabra provides a modified Reynolds number to represent the drag coefficients and wall effects in a shear-thinning polymer, which is in good accordance with experimental observations [86].

Magnetically assisted settling of materials are not well documented, as most applications involving microwires require flow alignment to create anisotropic properties in the material [87] [88], and hence the collection of fibers in a specific location is undesirable. The magnetically assisted settling of magnetic fibers, particularly in stagnation point flow $^{5}$, is a gap in literature which this thesis aims to fill.

\footnotetext{
${ }^{5}$ Stagnation point flow is the flow of fluid around a solid surface where the fluid is divided into two streams.
} 


\section{Chapter 3}

\section{Experimental Methodologies}

This chapter details the experimental methodologies used in the analysis of fiber motion. The selection and properties of magnetic fibers and suspension fluids are described in sections 3.1 and 3.2 respectively. The experimental setup for the electrical measurement of fiber rotation and translation is then presented. Optical methods used in the verification of the electrical measurements are discussed in Section 3.4. Finally, the experimental procedures are described.

\subsection{Selection and Properties of Magnetic Fibers}

In order to create porous ceramics, a technique where fibers are aligned in a magnetic field has been devised. The selection of fibers is dependent on geometric constraints and the fibers magnetic properties. The intended application for this research is in the development of ultrasonic transducers, where the wafer thickness is $1-2 \mathrm{~mm}$. A pore depth:diameter ration between 20 and 240 has been demonstrated to act as waveguides [89]. For this reason the fiber length used to create pores will have a length of $1 \mathrm{~mm}$, allowing for fiber diameters between 4 and $50 \mu \mathrm{m}$. The density of pores in the wafer is dependent on fiber concentration, where fibers with a lower diameter allow for a greater number of pores in the wafer compared to larger fiber diameters. The fibers are required to have a high magnetic susceptibility and low saturation magnetisation, such that the 
fibers can be easily manipulated in low strength magnetic fields. Finally, the fibers are required to be able to be burned out of the ceramic during the ceramics firing process in order to produce the desired pore.

Porous ceramics have been fabricated using an alumina-carbon mixture, where the carbon is burned out during the sintering process [90]. This suggests carbon may be a good material for creating aligned pores in ceramics, however carbon in all forms is usually diamagnetic [91]. To overcome the diamagentic nature of carbon materials, a magnetic material such as iron [66] [92] or nickel [93] [94] [95] is attached to increase the magnetic susceptibility. The sintering process reaches temperatures of up to $1530^{\circ} \mathrm{C}$ [90], which is sufficient to melt nickel but not iron, which have melting points of $1455^{\circ} \mathrm{C}$ and $1538^{\circ} \mathrm{C}$ [96] respectively. However the process in development at Callaghan Innovation has a sintering temperature of $1250^{\circ} \mathrm{C}$. At this temperature the carbon fiber will burn out of the ceramic and both iron and nickel will oxidise in the air.

From these considerations, a $500 \mathrm{~g}$ bag of precision-chopped nickel coated carbon fibers with a diameter of $7.12 \mu \mathrm{m}$ and a $1 \mathrm{~mm}$ length were sourced from Conductive Composites [97]. The chopping process used by Conductive Composites is a proprietary process which produces fibers that have exposed carbon on both end faces, as if lengths of carbon fiber are chopped after the nickel coating is applied. The nickel coating is applied via chemical vapor deposition which results in a higher quality coating on the fiber compared to electroplating techniques, illustrated in Figure 3.1.

Measurements of fiber lengths were obtained though taking scanning electron microscope micrographs of a sample size of 240 fibers and individually measuring the lengths of completely visible fibers. The fiber length distribution illustrated in Figure 3.2 shows a bimodal length distribution. Conductive Composites precision chopping has resulted in whole fibers with a length of $0.98 \pm 0.1 \mathrm{~mm}$, with $87 \%$ of measured fibers within this range. Short fibers with a length of $0.15 \pm 0.1 \mathrm{~mm}$ constitute $12 \%$ of the bulk fibers. These short fibers are a product of fibers breaking in either manufacturing, transport, or storage. The remaining $1 \%$ of fibers are between these modes and are fibers which have 

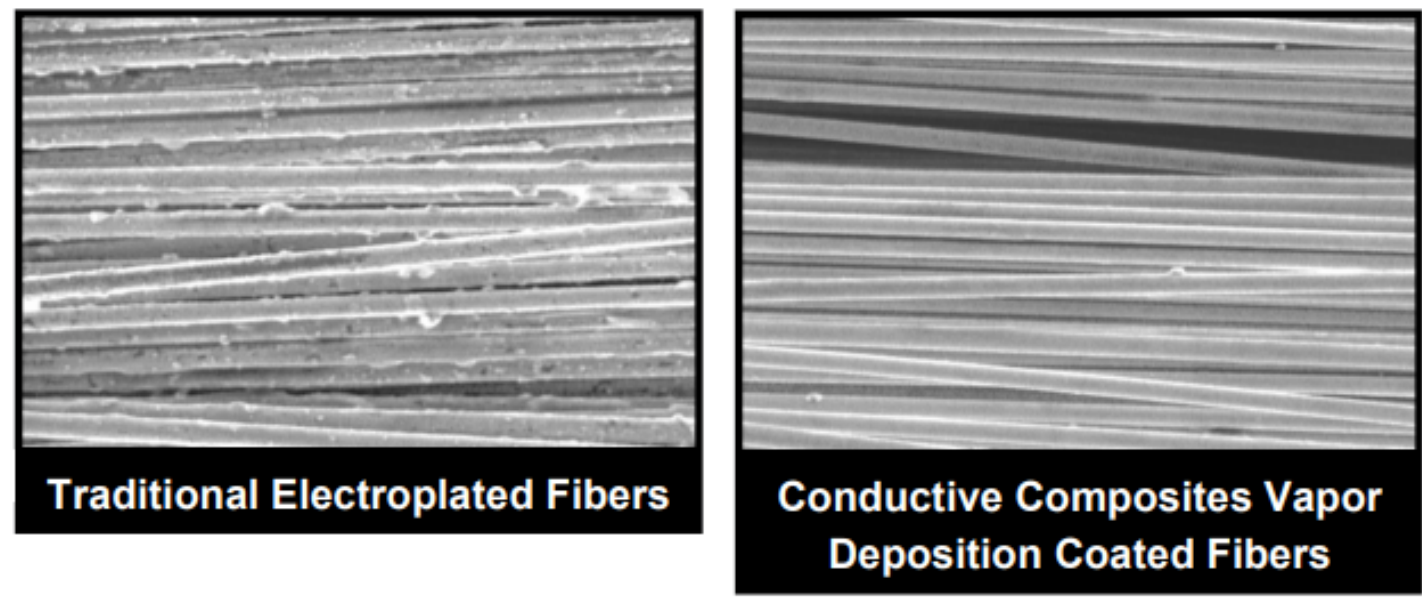

Figure 3.1: Chemical vapor deposition used in the application of a nickel coating to carbon fibers results in a more uniform layer when compared to electroplating techniques [98].

broken through the middle.

Damage to a bundle of fibers is illustrated in Figure 3.3, where the coating appears to have both split on the underside of the topmost fiber and caught on an object leading to the coating scrunching up. The other fibers in this bundle have similar scrunching damage and peeling to the nickel coating. The extent of the damage to the nickel coating is minimal, effecting only approximately $3 \%$ of the fibers observed in SEM images. The combination of shorter fibers and damaged fibers are expected to have negligible effects to the results for electrically measured fiber rotation due to the robust experimental design discussed in Sections 3.3 and 3.4, however it may impact the fabrication of porous ceramics.

Simon Granville of the Robinson Research Institute measured the magnetisation of the fibers using a superconducting quantum interference device (SQUID), with the results illustrated in Figure 3.4. The fibers were found to be too small to measure a single fiber in the SQUID, so a line of 30 fibers laying end to end was used to measure the magnetisation of a 'wire' of the fiber material, and the wire magnetisation was scaled to represent a single fiber. The hysteresis curve shows a saturation magnetisation of $2.2 \pm 0.1 \times 10^{-5} \mathrm{emu}$, however 


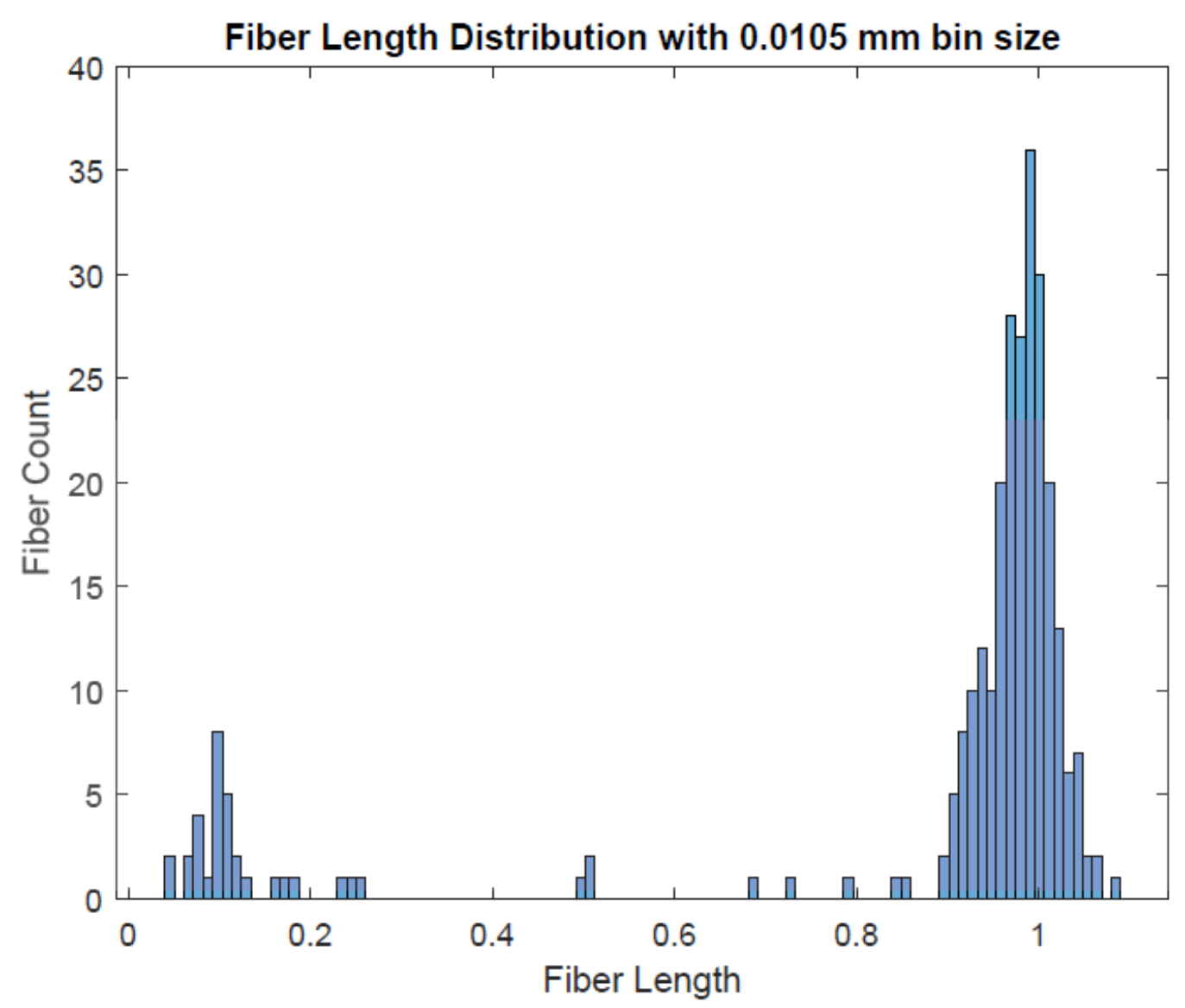

Figure 3.2: The fiber length distribution shows $87 \%$ of fibers have a length of $0.98 \pm 0.1 \mathrm{~mm}$. There are also a small number of fragments with a length of $0.15 \pm 0.1$ which constitutes $12 \%$ of the fiber lengths. 


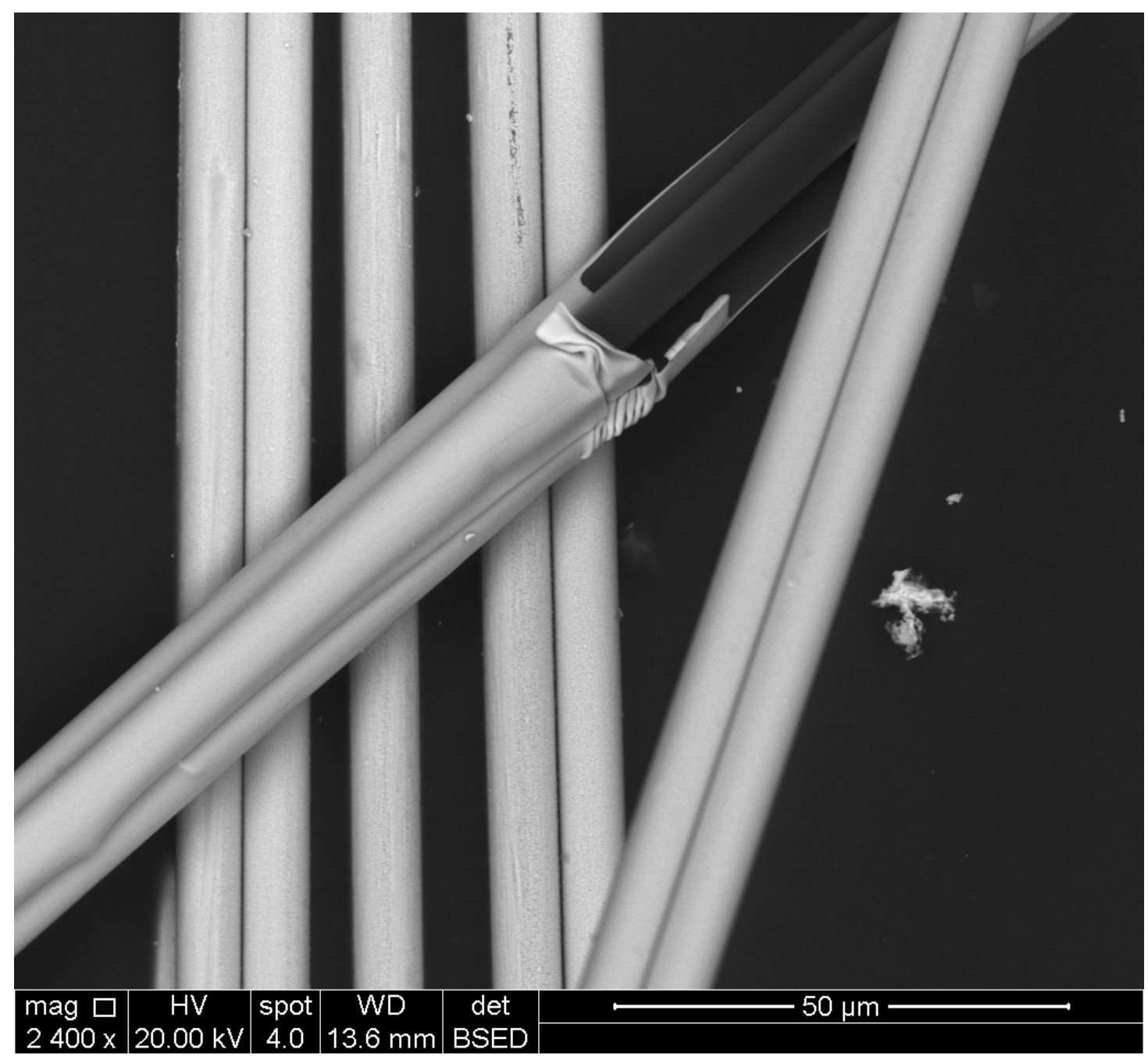

Figure 3.3: SEM photography shows how the nickel coating of one bundle containing 3 fibers appears to have split and compressed, resulting in an incomplete coating of the carbon fiber. 
$\mathrm{MH}$ at $300 \mathrm{~K}-30 \mathrm{Ni}$ coated fibres -24.07 .18$

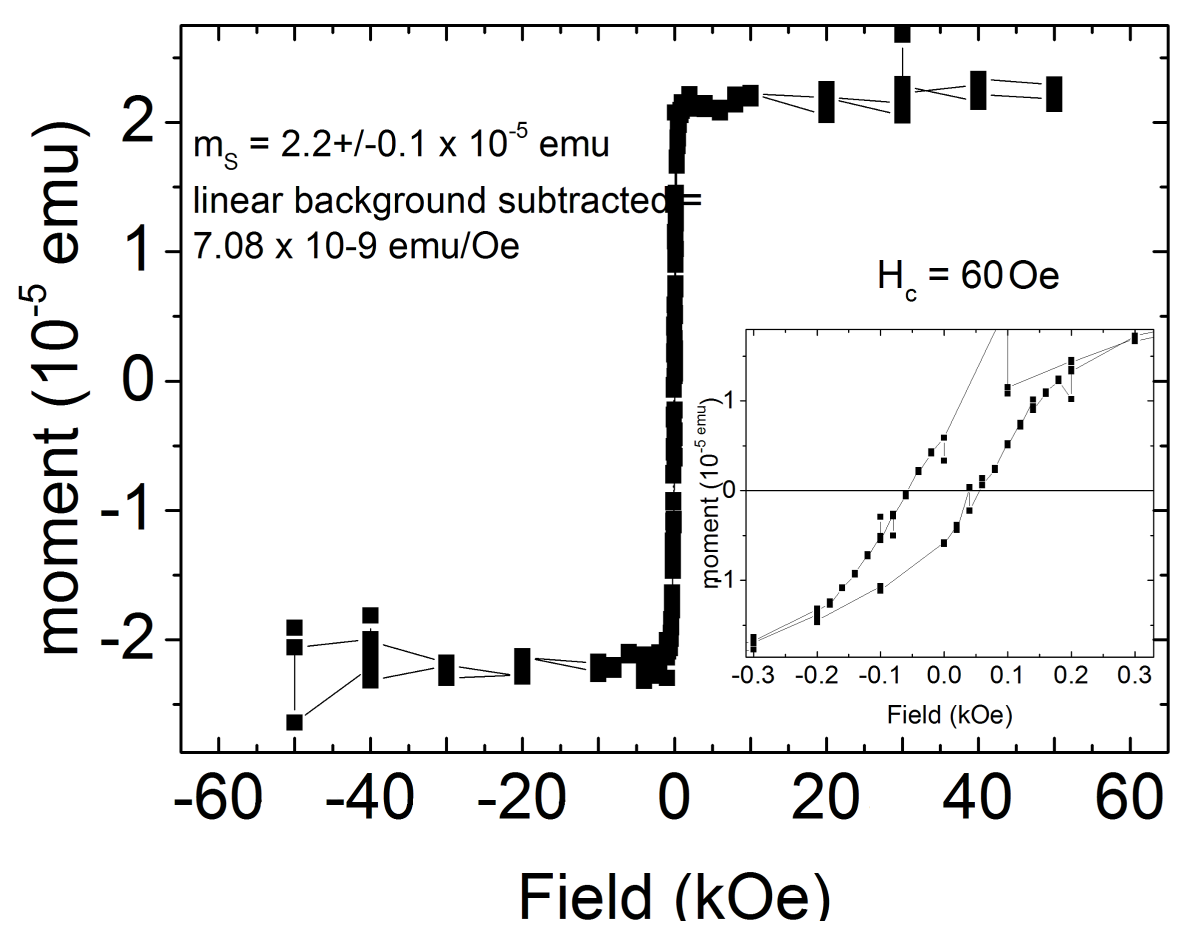

Figure 3.4: The magnetic hysteresis curve for nickel coated carbon fibers. The magnetic saturation can be observed to be $2.2 \pm 0.1 \times 10^{-5} \mathrm{emu}$ at fields greater than $0.3 \mathrm{KOe}(30 \mathrm{mT})$.

for the nickel magnetisation values reported in literature [99], we expect a saturation magnetisation of $4.91 \times 10^{-5} \mathrm{emu}$. This suggests the fibers have half as much nickel as stated by the supplier. The coercivity field strength required to demagnetise the fibers was measured to be $6 \mathrm{mT}^{1}$. The fibers reach magnetic saturation with an applied field of $30 \mathrm{mT}$.

The electrical measurement of fiber dynamics in a fluid solution detailed in Section 3.3 relied on the conductivity of the fibers being measurably greater than that of the fluid the fibers are suspended in. The resistivity of carbon in graphite form is $7.837 \mu \Omega \mathrm{m}$ giving a conductivity of $0.1276 \mathrm{MS} / \mathrm{m}$. The resistivity of nickel is $0.699 \mathrm{n} \Omega \mathrm{m}$, giving a conductivity of $14.3 \mathrm{MS} / \mathrm{m}$. The carbon

\footnotetext{
${ }^{1} 10000$ oersteds $=1$ tesla
} 


\begin{tabular}{|c|c|}
\hline Parameter & Value \\
\hline Length & $1 \pm 0.1 \mathrm{~mm}$ \\
Fiber Mass & $8.38 \times 10^{-11} \mathrm{~kg}$ \\
Diameter & $7.12 \mu \mathrm{m}$ \\
Fiber Density & $1800 \mathrm{~kg} / \mathrm{m}^{3}$ \\
Carbon Conductivity & $1.276 \times 10^{5} \mathrm{~S} / \mathrm{m}$ \\
Nickel Conductivity & $1.43 \times 10^{7} \mathrm{~S} / \mathrm{m}$ \\
Magnetic Saturation & $2.2 \times 10^{-5} \mathrm{emu}$ \\
Magnetic Moment & $7.43 \times 10^{-10} \mathrm{~A} / \mathrm{m}^{2}$ \\
Coercive Field & $6 \mathrm{mT}$ \\
Saturation Field & $30 \mathrm{mT}$ \\
\hline
\end{tabular}

Table 3.1: Summery of the properties of nickel coated carbon fibers

conductivity is expected to have a minimal contribution to the electrical measurement of fiber rotation due to the drastic difference between nickel and carbon conductivity. Furthermore, the high conductivity of nickel allows for the use of a number of suspension fluids discussed in Section 3.2. A summary of the fiber properties is presented in Table 3.1.

The fibers are stored in large bags containing $500 \mathrm{~g}$ of fibers resulting in a portion of the fibers stuck together. A procedure has been adopted to prepare the fibers for experimental use:

- A small quantity $(10-15 \mathrm{~g})$ of fibers were removed from the bulk and suspended in deionised water.

- The suspension is manually shaken until the bulk of the fibers have visibly separated (approximately 5 minutes).

- A small quantity of the fiber suspension is diluted and shaken until $80 \%$ of the fibers are separated.

- The dilute suspension is sonicated until the remaining fibers have visually separated. 
- The dilute suspension is manually shaken and then left to settle for 2 minutes. The unsettled fibers are decanted into another container, and this decanting process is repeated.

- The suspension is dried in glass container on a hot plate at $100^{\circ} \mathrm{C}$ until the liquid has evaporated.

The manual agitation of fibers results in a high number of interconnected bundles of fibers separating into the individual fibers, illustrated in Figures 3.5a and $3.5 \mathrm{~b}$. The decanting process removes the fibers which are stuck together and too small to be visually distinguished from the bulk separated fibers. The result of fiber separation after the fibers are dried is illustrated in Figure 3.5c, and the separated fibers in a transparent fluid suspension is illustrated in Figure $3.5 \mathrm{~d}$.

\subsection{Selection of a Suspension Fluid}

The development of techniques to monitor the motion of fibers subject to a magnetic field requires a aqueous solution to model the characteristics of a PZT slurry. Due to the opaque nature of PZT and its limited shelf life, it is inappropriate to use PZT itself. PZT slurrys can be designed to exhibit Newtonian behavior [100] however they are typically non-Newtonian [101] [102] and exhibit a psudoplastic sheer thinning behavior. Hence a transparent psudoplastic solution would be ideal as a model for PZT.

Carbomer $(\mathrm{C} 3 \mathrm{H} 4 \mathrm{O} 2)_{n}$ dispersions have been observed to exhibit this psudoplastic behavior [103] and can be manufactured to be transparent [104]. However, the preperation of these carbomers requires the rehydration of the carbomer powder in rapidly agitated water where the resulting viscosity is a function of the shear-stress during this process [105]. Coupled with the an observed change in viscosity with temperature [106], these factors make the creation of dispersions with accurate and highly repeatable viscosities difficult.

Instead of creating a pseudoplastic suspension fluid, it may be simpler to 


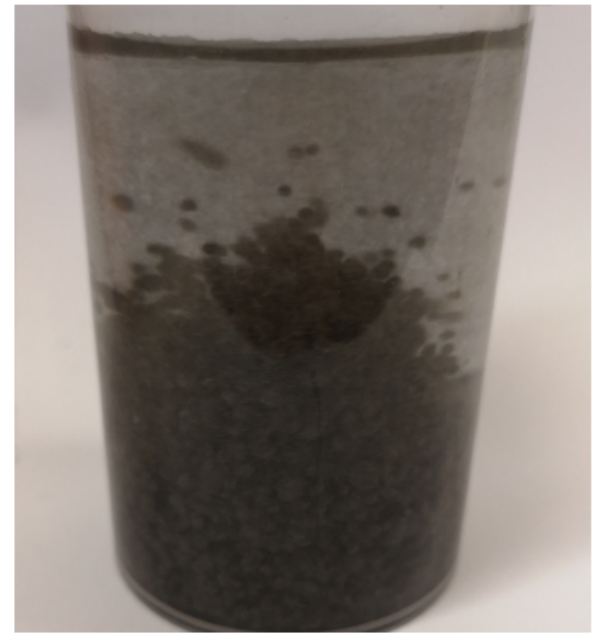

(a)

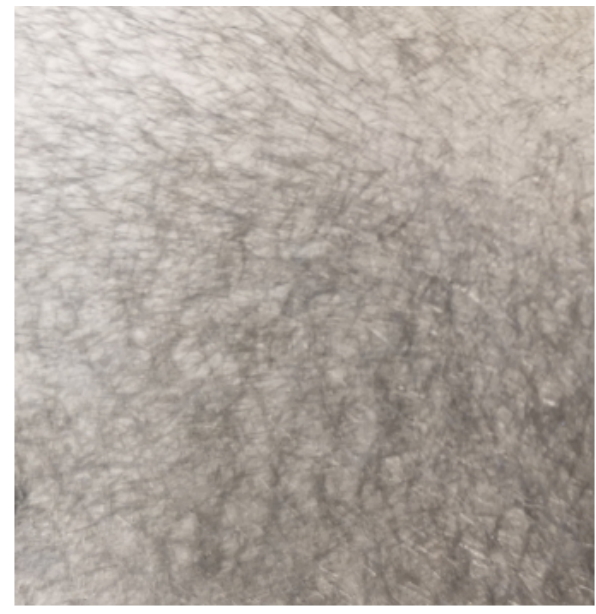

(c)

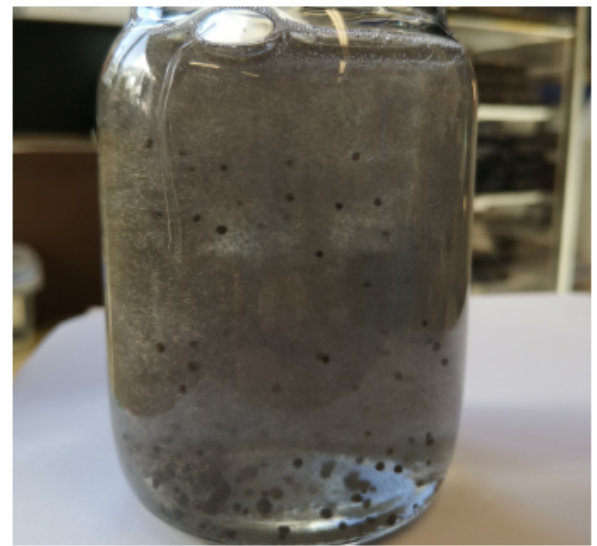

(b)

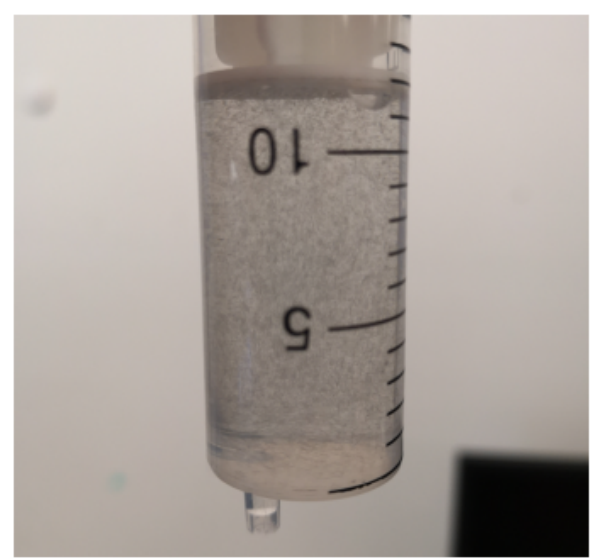

(d)

Figure 3.5: Fibers are separated from the bulk bag and suspended in deionised water (a). After manual agitation the bundles of fibers separate (b). Further manual agitation, sonication and decanting separates the remaining fibers. Evaporating the water leaves a mat of fibers (c) which are added to a fluid for experimental use $(d)$. 
create a PZT Newtonian ceramic slurry. The recipe for PZT piezoceramic can also be varied to maximise the piezoelectric effect so matching an exact suspension fluid to the slurry is inappropriate at this stage of the project. Instead, it is much more beneficial to use a generic Newtonian suspension fluid which has a controllable viscosity range over the expected viscosity of a PZT slurry. This range is between 10 and $1200 \mathrm{mPas}$ [107] [108].

A combination of glycerol $\left(\mathrm{C}_{3} \mathrm{H}_{8} \mathrm{O}_{3}\right)$ and deionised water were used to create a suspension fluid with a well defined viscosity. Glycerol is a Newtonian fluid, and hence does not exhibit the shear-thinning property observed in most pseudoplastic PZT dispersions. Glycerol is a colorless viscous water soluble liquid, has both well defined viscosity [109], electrical conductivity, and is weakly diamagnetic [110]. These properties make glycerol a suitable suspension fluid for both optical and electrical measurement of fiber dynamics. Additionally, glycerol has been used as a suspension fluid to observe the motion of fibers subject to a magnetic field [13]. The fluid viscosity we will be using in these experiments are 30,50 and $90 \mathrm{mPas}$ at room temperature. To achieve these viscosities $75 \mathrm{wt} \%, 80 \mathrm{wt} \%$, and $85 \mathrm{wt} \%$ will be used at a temperature of $22{ }^{\circ} \mathrm{C}$. This temperature and fluid density was derived from Table 3.2. The conductivity of glycerol-water solutions is dependent on the water content. The conductivity for the solutions we will be using is $5.8,4.25$, and $3.0 \times 10^{8} \mathrm{~S} / \mathrm{m}$ for our solutions respectively [110].

\subsection{Experiment Setup for the Electrical Measure- ment of Fiber Translation and Rotation}

A non-optical technique is required to measure the fiber dynamics in opaque PZT dispersions. This limits us to magnetic and electrical techniques. The ferromagnetic materials used in the construction of the magnetic cells discussed in Section 3.3.4 makes it difficult to accurately monitor the fiber dynamics due to magnetic interference. This leaves electrical techniques, of which monitoring the conductance over time is a simple approach. The alignment of fibers relative 


\begin{tabular}{|c|c|c|c|c|c|c|}
\hline & \multicolumn{7}{|c|}{ Temperature, ${ }^{\circ} \mathrm{C}$} \\
\hline Glycerol, wt $\%$ & 0 & 10 & 20 & 30 & 40 & 50 \\
\hline 0 & 1.792 & 1.308 & 1.005 & 0.8007 & 0.6560 & 0.5494 \\
10 & 2.44 & 1.74 & 1.31 & 1.03 & 0.826 & 0.680 \\
20 & 3.44 & 2.41 & 1.76 & 1.35 & 1.07 & 0.879 \\
30 & 5.14 & 3.49 & 2.50 & 1.87 & 1.46 & 1.16 \\
40 & 8.25 & 5.37 & 3.72 & 2.72 & 2.07 & 1.62 \\
50 & 14.6 & 9.01 & 6.00 & 4.21 & 3.10 & 2.37 \\
60 & 29.9 & 17.4 & 10.8 & 7.19 & 5.08 & 3.76 \\
65 & 45.7 & 25.3 & 15.2 & 9.85 & 6.80 & 4.89 \\
70 & 76.0 & 38.8 & 22.5 & 14.1 & 9.40 & 6.61 \\
75 & 132 & 65.2 & $\mathbf{3 5 . 5}$ & $\mathbf{2 1 . 2}$ & 13.6 & 9.25 \\
$\mathbf{8 0}$ & 255 & 116 & $\mathbf{6 0 . 1}$ & $\mathbf{3 3 . 9}$ & 20.8 & 13.6 \\
$\mathbf{8 5}$ & 540 & 223 & $\mathbf{1 0 9}$ & $\mathbf{5 8 . 0}$ & 33.5 & 21.2 \\
90 & 1310 & 498 & 219 & 109 & 60.0 & 35.5 \\
100 & 12070 & 3900 & 1412 & 612 & 284 & 142 \\
\hline
\end{tabular}

Table 3.2: Viscosity of Glycerol-water solutions as a function of temperature [109].The fiber viscosities we will be using for experiments are 30, 50, and 90 mPas. 
to an electrode is expected to have a significantly different conductivity to that of randomly aligned fibers due to the conductivity of the nickel creating a path with reduced resistance between the anode and cathode of the electrodes.

In order to measure conductivity over time, the system presented in Figure 3.6 will be used. The lock-in amplifier is connected to an electrode set and is used to measure the small signal changes in current associated with fiber motion through the sensing region. A switch unit (switching multiplexer) adds spatial diversity of the system at the cost of temporal resolution though time division duplexing whereby each electrode is measured for discrete blocks of time. An external field provided by either a solenoid or a permanent magnet is used to manipulate the position and orientation through creating a linear field in the center of a solenoid, or maximising the flux density from a permanent magnet using the magnetic cell. A thermocouple is used to measure the temperature of the fiber suspension to calculate the specific viscosity throughout the experiment.

\subsubsection{Lock-in Amplifier}

A SR830 DSP lock-in amplifier is used to record the in-phase and out-of-phase component of the current across the electrodes. A lock-in amplifier has high sensitivity sensing with low noise which is crucial for measuring the change in conductance of the glycerol-water solution due to microwires. Additionally, the SR830 has a high sampling rate and variable reference frequency in conjunction with a low pass filter allowing for the removal of high frequency noise otherwise present in the system.

The lock-in amplifier can be easily integrated with data acquisition software to record and maintain in situ measurements which is a requirement of the experimental design.

The main disadvantage of a lock-in amplifier is the inability to quickly scan multiple frequencies. This is due to the lock-in amplifier having a single excitation frequency and a measurement taken at that frequency. For the fiber motion application of this thesis, this is not an issue as we are interested in the 


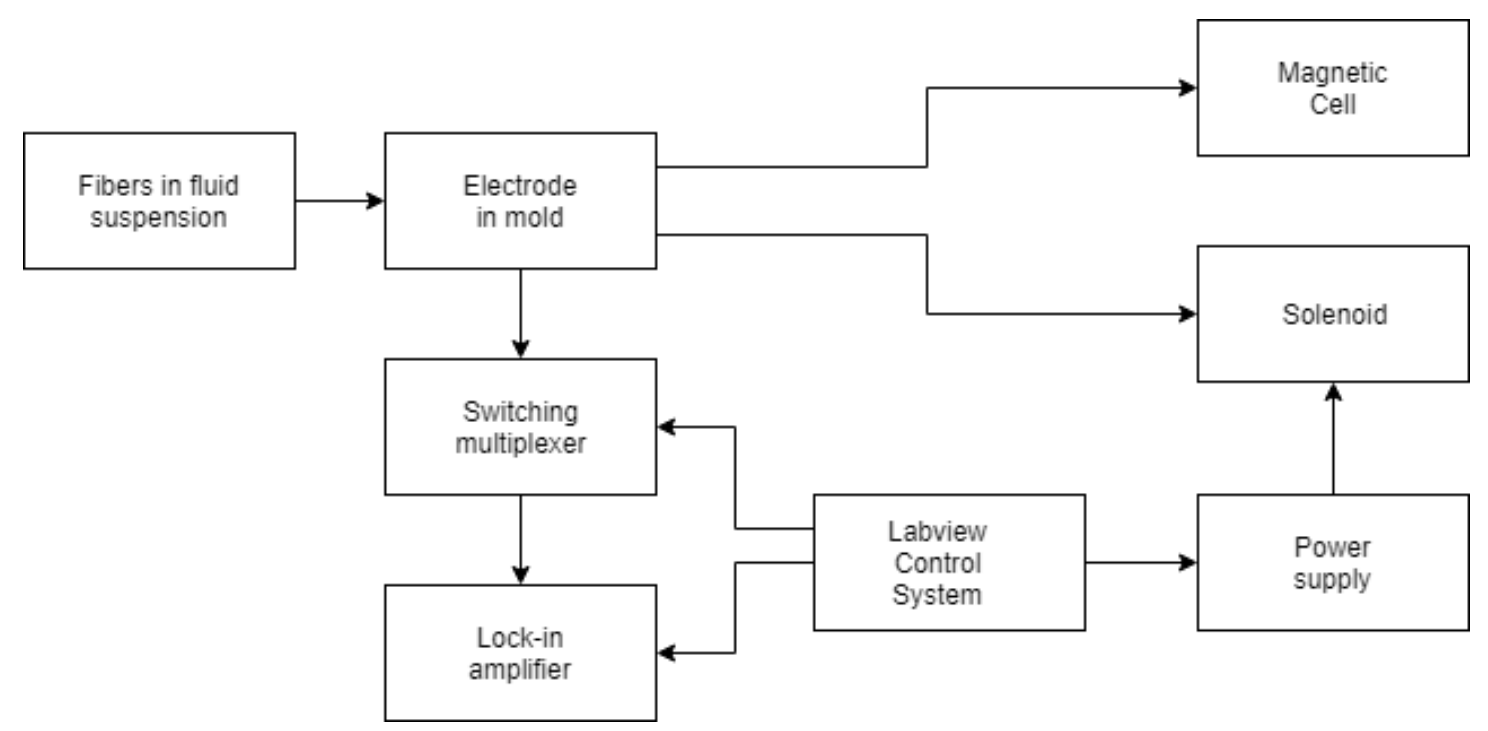

Figure 3.6: The fiber suspension is injected via a syringe into a mold containing an electrode. For fiber rotation experiments the electrode placed in a solenoid, whereas for translation experiments it is placed inside a magnetic cell. The electrode is connected to a switching multiplexor, which in turn is connected to a lock-in amplifier. 
relative change in conductance at a particular frequency and not across a wide frequency spectrum.

Alternatives to a lock-in amplifier are impedance analysers, which scan over a range of frequencies. These allow for the simultaneous analysis of high frequency $(\approx 10 \mathrm{GHz})$ and low frequency $(\approx 50 \mathrm{~Hz})$ components of the system. The disadvantage of this is the time taken to scan over a range of frequencies thereby limiting the temporal resolution.

\subsubsection{Multiplexor}

A Keysight 34970a switching multiplexor was used to interface between the lock-in amplifier and the electrode sets. This multiplexor has 8 controllable outputs allowing for a large number of electrodes to be simultaneously connected yet still isolated from the lock-in amplifier. The multiplexor outputs are isolated through relays which have minimal switching noise resulting in minimal distortion and noise introduced to the system.

\subsubsection{Electrode configurations}

The sensing of fiber alignment and position has been achieved using three electrode configurations. These rely on the suspension fluid having a lower conductance than the fibers, which is a potential limitation of the sensing design for wider applications. Given that glycerol-water solutions satisfy this condition, the conductance measured by the lock-in amplifier is a function of fiber orientation and density. The geometrical properties of the electrodes dictate whether the current will increase or decrease with a change in orientation and density. The electrode plates are printed in gold plated copper on a flexible glass-reinforced plastic sheet with an earth plate on the reverse side. All electrode plates are $5 \mathrm{~mm}$ wide and separated vertically by either a 2 or $5 \mathrm{~mm}$ gap. 


\section{Quadrant Electrode Configuration}

A quadrant electrode configuration illustrated in Figure 3.7a senses the fiber density and orientation horizontally across the mold. This results in a high conductivity for fibers randomly aligned as the conduction path is shortened by the fibers. The application of a magnetic field results in the fibers aligning parallel to the electrode and shortening the conduction path, which decreases the conductivity of the glycerol-water suspension. The act of measuring across the electrode creates an oscillating electric field, which induces a small AC magnetic field orthogonal to the driving field from the solenoid due to charge displacement. Due to the orthogonality of these fields, it is possible the small AC magnetic field to dominate the motion in fibers close to the horizontal axis. However due to the magnitude difference between these magnetic fields, fibers do not commonly settle at the angle at which the AC magnetic field dominates.

\section{Ring Electrode Configuration}

The implementation of a ring electrode, illustrated in Figure 3.8a, allows for the net orientation and density of fibers in a glycerol-water solution to be implicitly measured. This operates similar to the quadrant electrode, however the electric field and sensing region is a hemispherical against the side of the electrode. This results in a longer conduction path for randomly aligned fibers compared to aligned fibers. As with the quadrant electrode configuration, the charge motion resulting from the oscillating electric field results in a small AC magnetic field. The AC magnetic field is parallel to the applied field hence the superposition of magnetic fields occurs. The resulting magnetic field provides a small increase in the net magnetic field and hence requires a lower magnetic field produced by the solenoid for magnetic saturation in the fibers.

\section{Ladder Electrode Configuration}

The ladder electrode configuration illustrated in Figure 3.9a is an extension of the ring electrode with multiple pairs of ring electrodes stacked vertically in a 


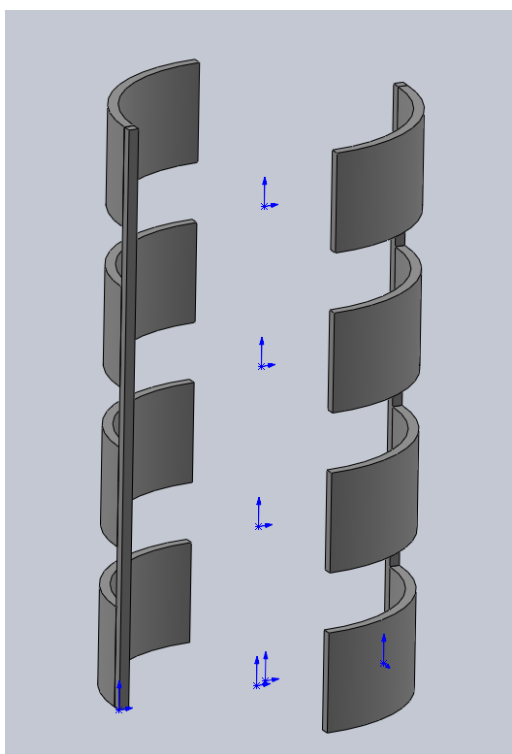

(a) 3D model of the electrode plates.

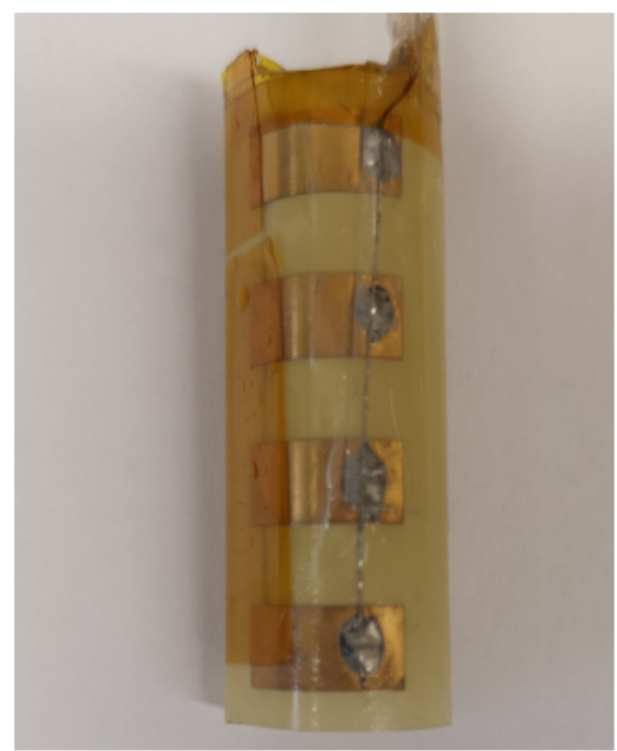

(b) Photograph of the quadrant electrodes

Figure 3.7: In a quadrant electrode configuration the each electrode encompasses a quarter of a cylindrical mold. Randomly aligned fibers create a lower resistance path between the electrodes compared to vertically aligned fibers. Each electrode plate is $5 \mathrm{~mm}$ high, and the cylinder has a diameter of $14 \mathrm{~mm}$. 


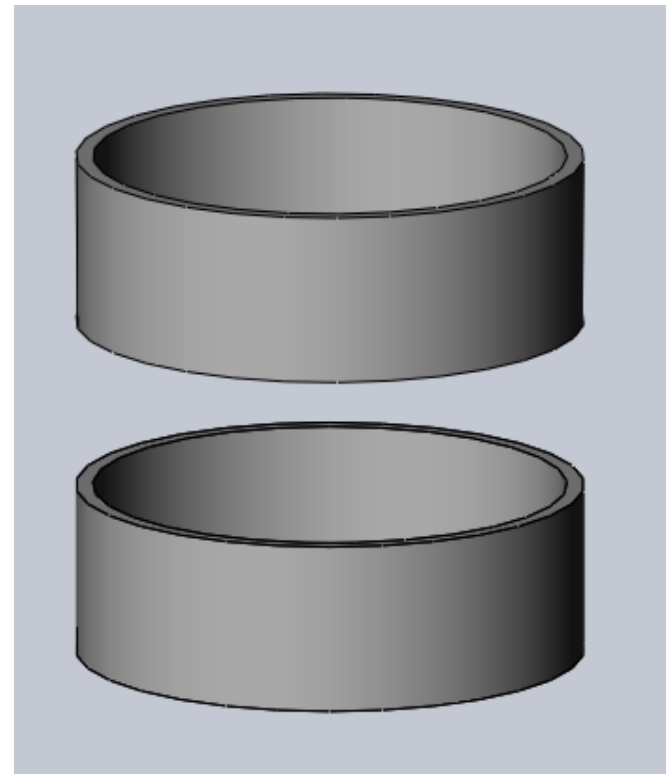

(a) 3D model of the ring electrode configuration.

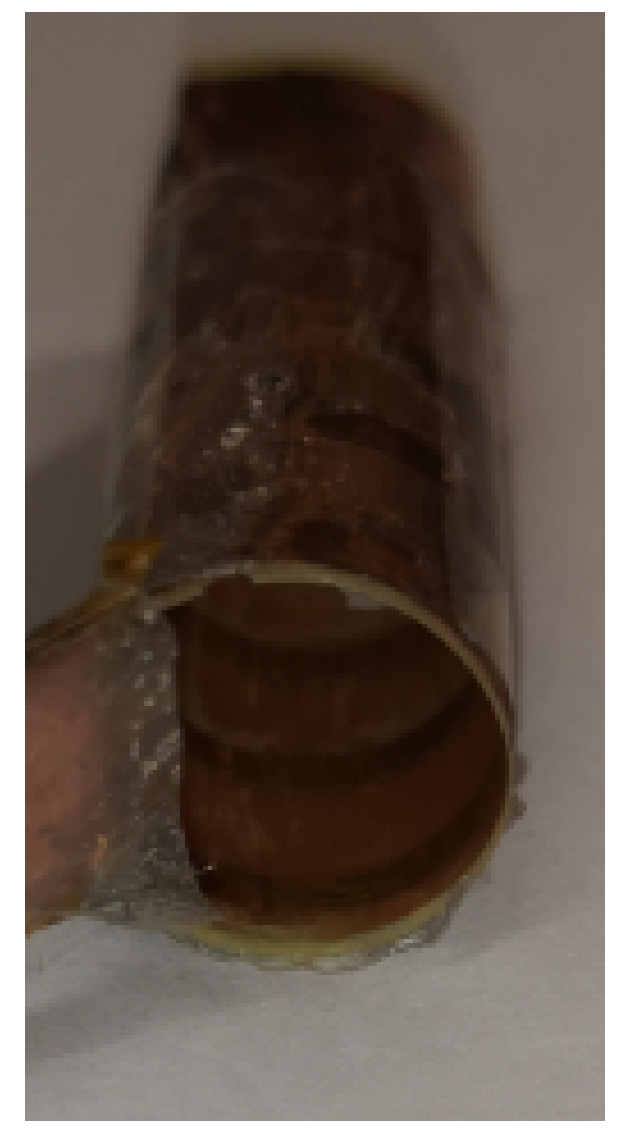

(b) Photograph of the ring electrodes

Figure 3.8: In a ring electrode configuration, each electrode consists of a ring encompassing the full circumference of a cylindrical mold. Each electrode plate is $5 \mathrm{~mm}$ high, and the cylinder has a diameter of $14 \mathrm{~mm}$. The electric field resulting from the lock-in amplifier and electrode plates are parallel to the magnetic field, hence superposition of these fields may occur. 
mold. Through using the anode of one electrode pair as the cathode of another a greater volume of the mold can be analysed compared to directly stacking multiple ring electrodes in the mold. The electrode's anode-cathode pairs are illustrated in Figure 3.9b. This spatial diversity afforded through this scheme removes the ability to simultaneously measure multiple regions of the mold, and a switching multiplexor is required to iterate through the electrode array. Aligned fibers setting to the bottom of the mold can be inferred using this system due to the conductivity differences between the glycerol-water solution and the fiber suspension. The electrode positions in the cell define the conductance we expect to measure during the experiment. An electrode at the bottom of the mold would result in increasing conductivity as fibers collect in the bottom of the mold, however the spatial density of fibers risks current saturation in the lock-in amplifier. An electrode placed around the length of a fiber $(1 \mathrm{~mm})$ from the bottom of the mold results in near-field interference around the electrode, resulting in the conductance measured by the bottom electrode differing significantly from the other electrodes. Finally, an electrode placed multiple fiber lengths from the bottom of the mold would not exhibit either current saturation or near-field interference, however the motion of fibers in the region of space underneath the electrode will not be detected. The electrode positioning we implemented is the latter, with the bottom electrode positioned $5 \mathrm{~mm}$ from the bottom of the mold. A series of 5 electrodes are spaced $5 \mathrm{~mm}$ apart vertically up the mold from the bottom electrode. The top electrode is $2 \mathrm{~mm}$ from the top of the mold.

\subsubsection{Magnetic Cells}

In order to maximise the magnetic flux density through the mold two magnetic cells were fabricated. The cells consist of a permanent magnet enclosed in a 1008 high carbon steel enclosure, illustrated in Figure 3.10. The spherical cell completely encloses the mold and fiber suspension and provides maximum magnetic flux density. In contrast the open sided cell allows for optical access however it has a lower magnetic field gradient. The minimum magnetic field 


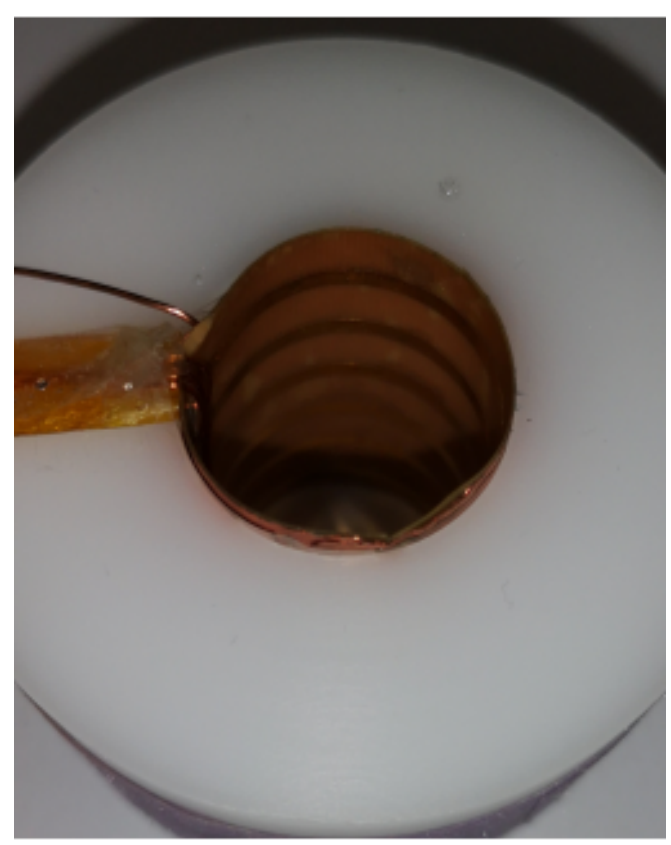

(a) Ladder electrode configuration.

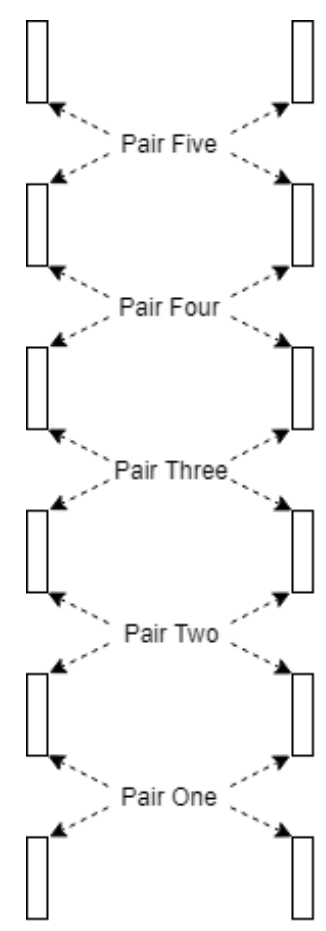

(b) Pairs of electrodes.

Figure 3.9: The ladder electrode is a series of ring electrode pairs stacked vertically inside a mold. Each electrode is used in two pairs except for the top-most and bottom-most electrodes. Each electrode plate is $5 \mathrm{~mm}$ high, and the cylinder has a diameter of $14 \mathrm{~mm}$. 
strength inside either cell is $80 \mathrm{mT}$, illustrated in Figure 3.11. The magnetic field in both cells are sufficient to put the fibers into magnetic saturation at all points inside the cells. The magnetic force acting on the fibers is a function of the magnetic field gradient, with high gradients resulting in higher forces. The magnetic field gradients are stronger close to the magnets, illustrated in Figure 3.12 , making fibers in this region settle faster relative to regions further from the magnet.

\subsubsection{Solenoid}

A solenoid can be used to create a uniform magnetic field through the application of a DC current. The magnetic field is given by Ampere's law,

$$
B=\mu_{0} n I
$$

where $B$ is the magnetic field strength at the center of a solenoid, $\mu_{0}$ is the magnetic constant, $n$ is the number of turns per meter, and $I$ is the current applied to the solenoid. The magnetic field created from air-cored solenoids is limited by resistive heating of the solenoid, creating both an unstable temperature and an upper limit to the applied current [111].

The solenoid used has 580 turns per meter which produces a magnetic field strength of $0.73 \mathrm{mT} / \mathrm{A}$. To generate a field sufficient to saturate the fibers the current through the solenoid is required to be $42 \mathrm{~A}$. This was achieved using a HP6031 power supply with a 0-20 V, 0-120 A output potential. The relative magnetic field inside the solenoid was measured using a search coil to assess the uniformity of the magnetic field, and it was found to have a maximum deviation of $5 \%$ over the length of the solenoid.

\subsubsection{Labview Software}

Control software was required to control the lock-in amplifier, switching multiplexor, and power supply, as well as record data from the lock-in amplifier and thermocouple. National Instruments Labview software is commonly used 


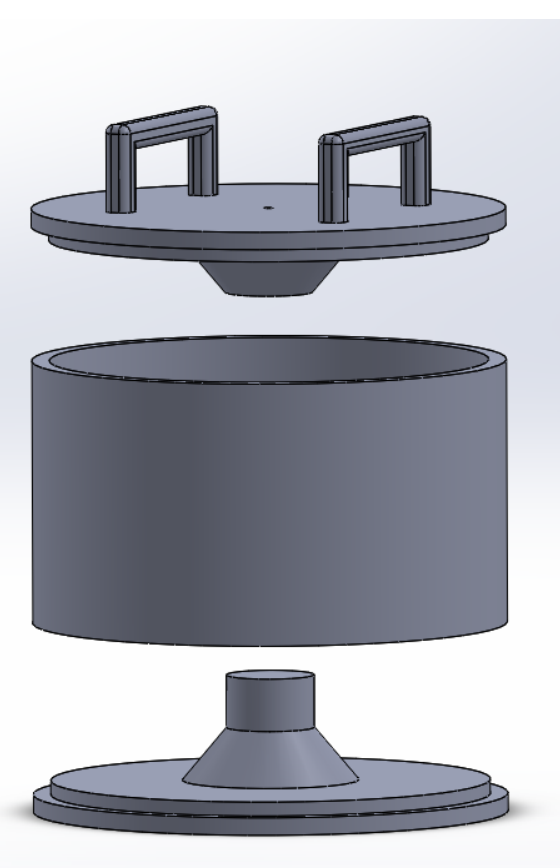

(a) Enclosed magnetic cell.

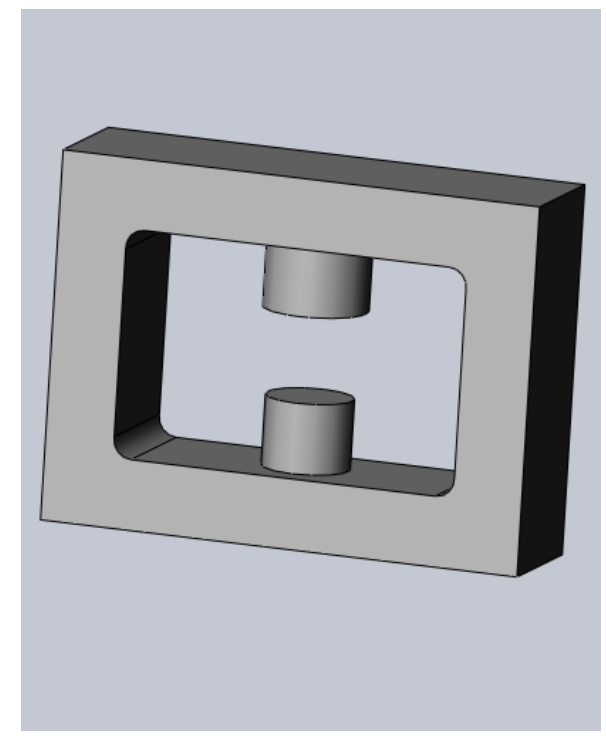

(b) Open magnetic cell

Figure 3.10: The enclosed magnetic cell (a) used for fiber settling is a 3 piece construction. The construction consists of a top and bottom with pole pieces which maximise the magnetic flux through the piezoceramic mold, and a middle wall segment which forms a complete cell. In contrast, the open magnetic cell (b) consists of one solid casing with a single pole piece which both allows optical access to the piezoceramic mold whilst improving the magnetic flux over a magnet alone. In both magnetic cells a permanent magnet is positioned on top of the bottom pole piece, which is used to generate the magnetic field. 


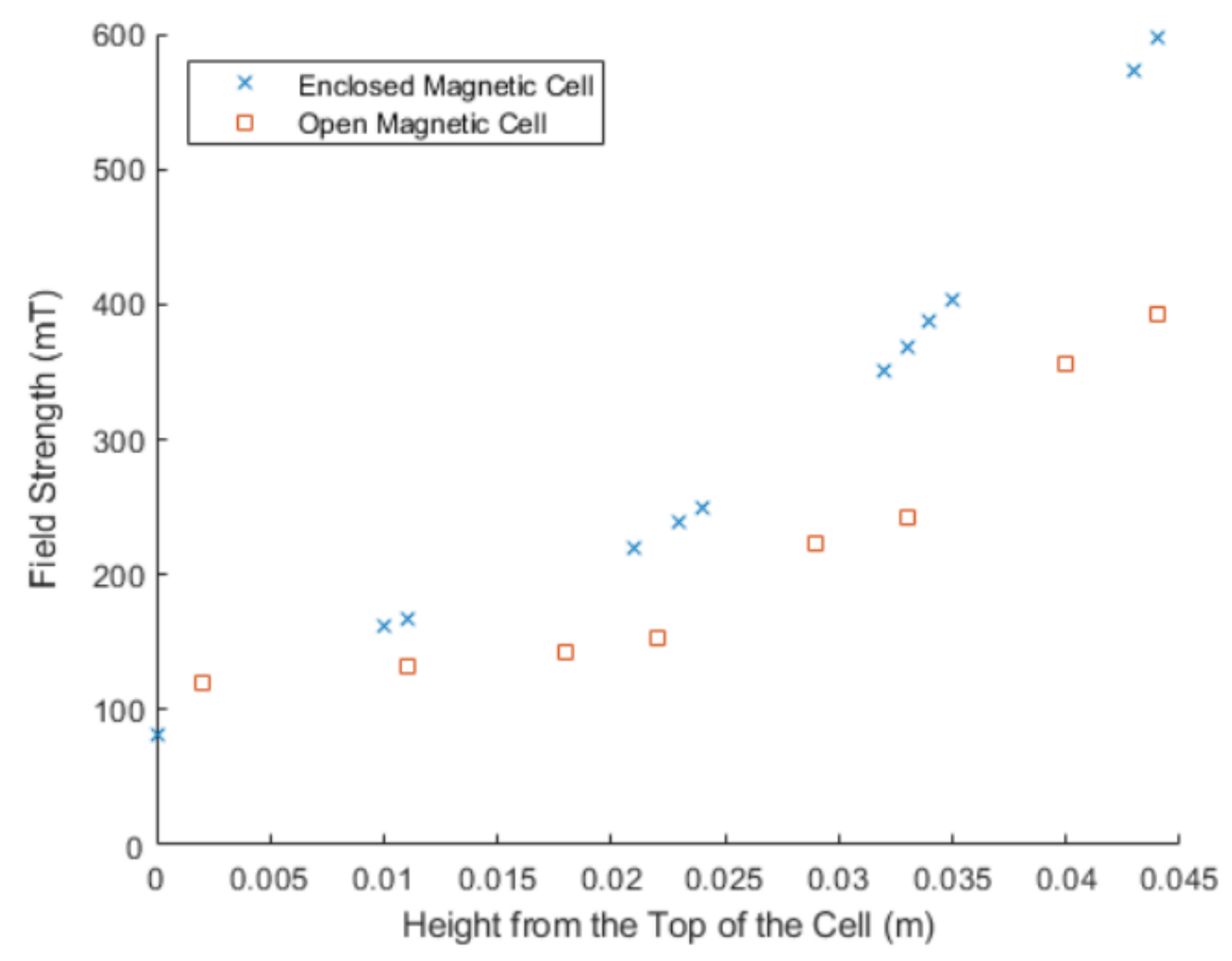

Figure 3.11: The magnetic field strengths in both the open and enclosed cells have a minimum magnetic field intensity at the face of the top pole piece $(z=0)$, however this minimum is sufficient to magnetically saturate the nickel coated carbon fibers. $\mathrm{z}=0$ denotes the face of the top pole piece, and $\mathrm{z}=0.045$ denotes the face of the bottom pole piece. 


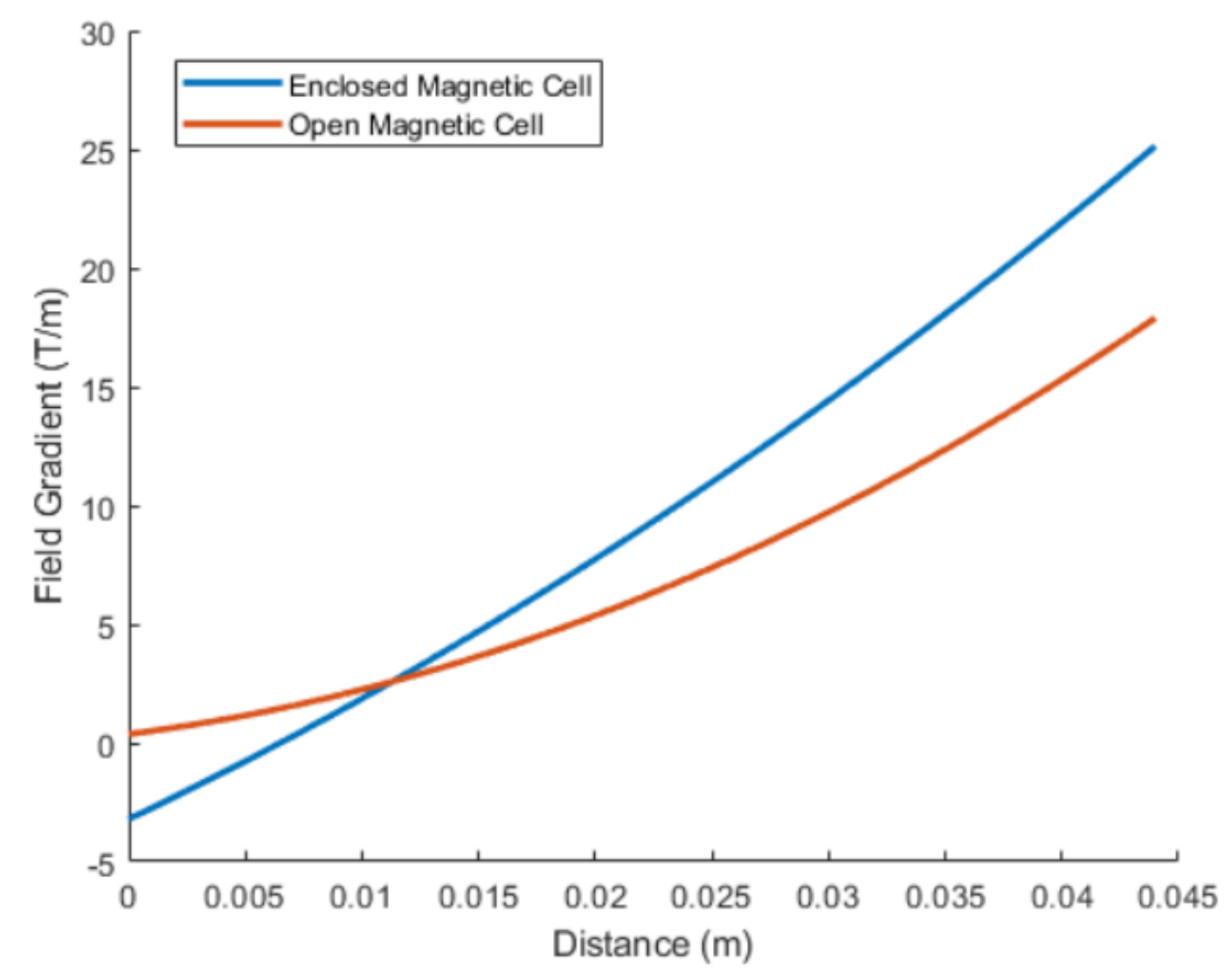

Figure 3.12: Interpolating and differentiating the magnetic field strengths inn each sell provides the field gradient. The magnetic field gradient in the enclosed magnetic cell is has negative region at the top of the cell, whereas the field gradient in the open magnetic cell is entirely positive. $z=0$ denotes the face of the top pole piece, and $\mathrm{z}=0.045$ denotes the face of the bottom pole piece. 
in both academic and industrial environments for data acquisition and instrument control, hence it was selected for this project.

The Labview software is a visual programming suite where a series of component blocks are connected in order to control connected instruments. The key component blocks are the 'VISA write' and 'VISA read' blocks, which when given an address for the specific instrument, writes or reads from the devices internal buffers and storage. Through these blocks the 4 instruments used in this thesis are simultaneously controlled and data is collected.

The control scheme for fiber rotation and translation experiments are very similar, with a few key differences. The Fiber rotation experiments involve applying current to a solenoid, hence the Labview script is required to control a power supply. However only a single electrode is measured throughout the experiment and so the multiplexor is not required to use the switching functionality. During settling experiments the solenoid is not required, hence the power supply is not used. However, a switching multiplexor is required to measure the conductance across an array of electrodes.

\subsection{Experimental Setup for the Optical Measure- ment of Fiber Translation and Rotation}

The goal of this project is to develop methods to measure the motion of fibers in a magnetic field using both optical and electrical techniques, with the intended application being the manufacturing of porous piezoceramics. Transmission optical microscopy is typically used for monitoring the rotation of magnetic fibers in a fluid suspension [13] [14], hence a modified version of this technique was implemented using a Helmholtz coil to generate a magnetic field, and a Basler camera to record the fibers motion. The combination of these two pieces of apparatus with a transparent mold containing the fiber suspension gives an optical measurement of fiber rotation to compare to electrical measurements. The open magnetic cell used in conjunction with the camera can be used to measure the magnetically assisted setting of fibers over time. 


\subsubsection{Helmholtz Coil}

A Helmholtz coil, illustrated in Figure 3.13, consists of two wire coils parallel to each other, which creates a uniform magnetic field between the coils. The magnetic field strength $B$ generated at the center of the Helmholtz coil is described by

$$
B=\left(\frac{4}{5}\right)^{3 / 2} \frac{\mu_{0} n I}{R},
$$

where $\mu_{0}$ is the magnetic constant, $n$ is the number of turns per meter, $I$ is the current applied to the solenoid, and $R$ is the radius of the coils. The Helmholtz coil used has 2410 turns per meter and a radius of $0.120 \mathrm{~m}$. This high turn density is achieved through having multiple layers of of coil on top of each other. The resulting magnetic field strength measured using a USB magnetometer is presented in Figure 3.14. Due to the power supply's current limit, the maximum obtainable magnetic field was $30 \mathrm{mT}$.

\subsubsection{Basler Camera}

The Basler camera, illustrated in Figure 3.15, was used to record the fiber rotation. This camera is capable of recording up to 300 frames per second (fps) in an uncompressed format, however is limited to a maximum file size of 3 GB. This frame-rate is far greater then we require for monitoring fiber dynamics. The ideal recording frame-rate for our purposes is a scalar multiple of the desired playback frame-rate, as this avoids inconsistent frame skipping. An example of frame rate skipping is a frame-rate of $90 \mathrm{fps}$ observed at $60 \mathrm{fps}$ resulting in skipping every third frame. A common frame-rate used in time-line scrolling and playback in video editors is $24 \mathrm{fps}$, so this was used as the recording frame-rate. This frame-rate has the additional benefit of maximising recording length due to the Basler camera's file size limitation. 


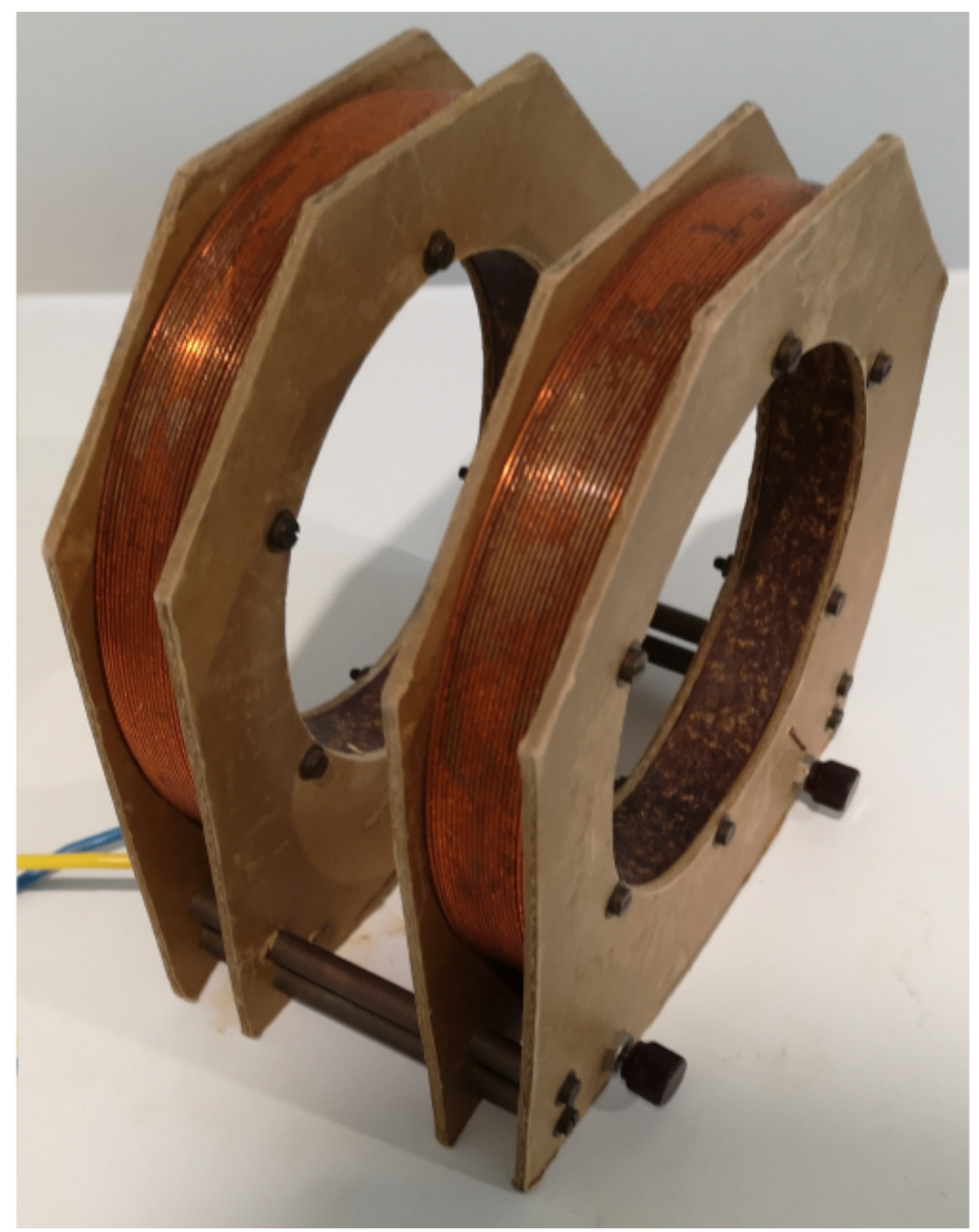

Figure 3.13: A Helmholtz coil consists of two coils separated by an air gap. A uniform magnetic field is generated between the coils through the application of current to the coil windings. 


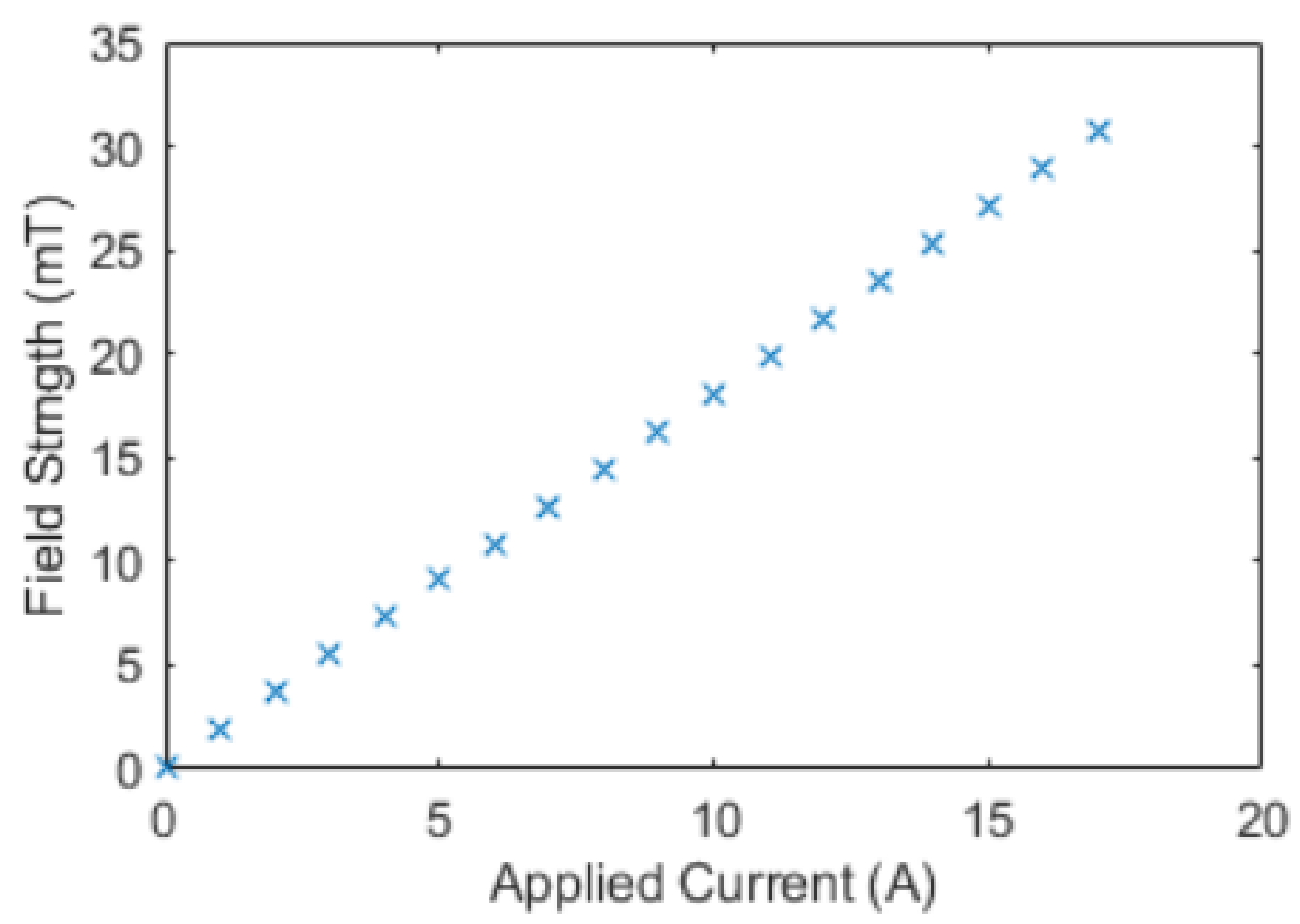

Figure 3.14: The magnetic field strength obtainable from the Helmholtz coil is linearly dependent on the applied current. The maximum applicable current limited by resistive heating through the coils. 


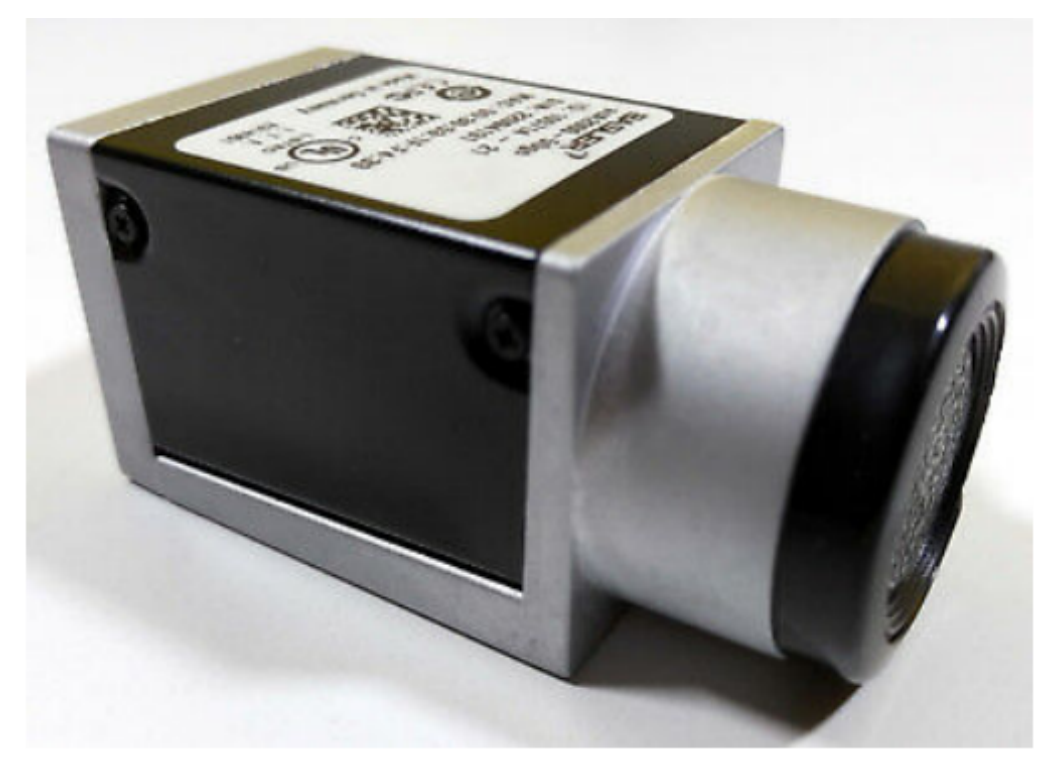

Figure 3.15: The Basler camera used to optically record the fiber dynamics can record up to $300 \mathrm{fps}$ in an uncompressed format, however it has a maximum recording size of $3 \mathrm{~GB}$.

\subsubsection{Transparent Fiber Mold}

The transparent mold was created to allow optical access to the fibers while they rotate in the magnetic field. The mold is required to be sufficiently large enough to allow fiber motion without interactions between fibers and the sides of the mold, yet be small enough to create a 2D plane of fibers and hence limit (but not completely remove) the potential for inter-fiber interactions. This mold was created using two glass slides separated by a $1 \mathrm{~mm}$ thick layer of silicone sealant, creating a $1 \mathrm{~mm} \times 15 \mathrm{~mm} \times 60 \mathrm{~mm}$ chamber to analyse fiber motion. This allowed for the injection of fibers between these slides and the subsequent optical analysis of a single layer of non-interacting fibers, as well as regions of interacting fibers in the same fame, without the fibers interacting with the glass slides. A separation distance of $0.6 \mathrm{~mm}$ was found to have a majority of fibers interacting with the mold walls. The mold was oriented vertically during the experiment, further limiting the potential for interaction with the glass slides due to gravitational setting over time. 
A second transparent container was used to monitor the magnetically assisted settling of the fibers. This container is a $10 \mathrm{~mm} \times 10 \mathrm{~mm} \times 44 \mathrm{~mm}$ square plastic container. This mold models the volume inside the molds used for the fabrication of piezoceramic molds, which are cylindrical with a diameter of 14 $\mathrm{mm}$.

\subsection{Experimental procedures}

In order to produce repeatable results for experiments with a large range of variables a set of defined experimental procedures are required. Firstly, fluid suspension viscosity has a large effect on fiber motion hence the creation of specific fluid viscosities is required. The application of the suspensions to the sensing apparatus can create anomalous effects including air bubbles in the fluid and a non-uniform fiber concentration throughout the fluid. An experimental procedure was created to mitigate these potential sources of error. Finally, the optical experiments are required as a base model to compare the results against, hence it is of critical importance to have a well defined experimental control.

\subsubsection{Fiber Suspension Preparation}

In order to create a fluid suspension with a specific viscosity and fiber concentration, a small amount of fibers (8-12 $\mathrm{mg}$ ) were placed inside a plastic container and weighed on a microbalance. The precision of the microbablance is $0.1 \mathrm{mg}$, giving a $1 \%$ error in the number of fibers. The corresponding glycerol weight is then added to the fibers via a large volume syringe. Finally, the deionised water is added via a small syringe. The suspension is agitated in order to combine the glycerol and water, and create a uniform fiber distribution. The resulting fiber suspension has an error in viscosity of $\pm 2 \mathrm{mPas}$ due to the inaccuracy in the microbalance and human induced error through adding slightly too much glycerol or water. 


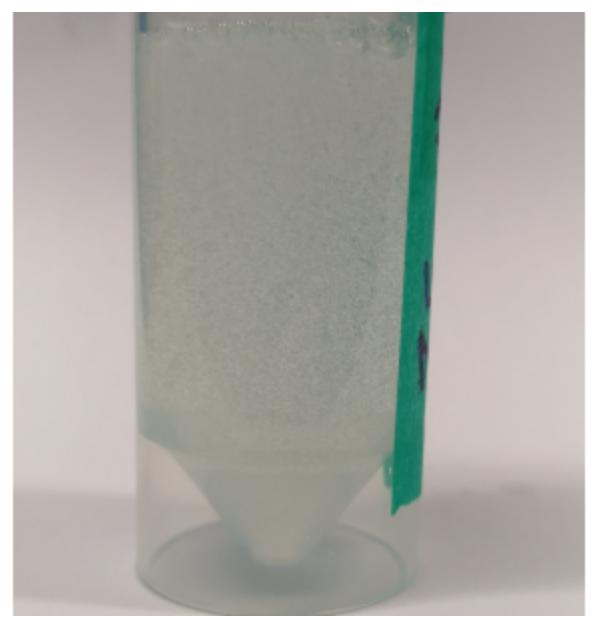

(a)

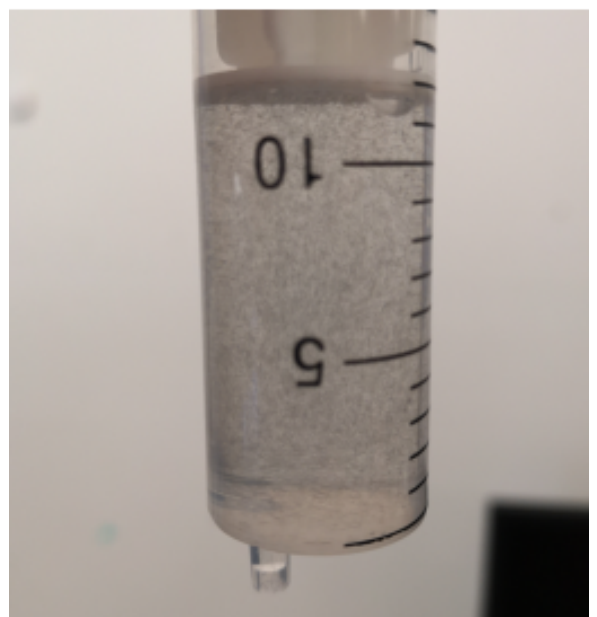

(b)

Figure 3.16: Agitating the fiber suspension to uniformly distribute fibers results in air bubbles in the fluid (a) which are removed thorough sucking the fluid up into a syringe $(b)$.

\subsubsection{Electrical Experiments}

The accurate measurement of fiber rotation in the solenoid requires an even fiber distribution and no air bubbles in the mold. Additionally, the conductance of the unaligned fiber suspension is required to observe the change of conductance due to short term gravitational effects. Due to the hysteresis effect discussed in Section 4.3.2, fibers are discarded after a single experiment. This simplifies the experimental procedures as the gravitational settling of fibers over extended periods of time can be ignored, and a uniform fiber distribution throughout the sensing apparatus can be obtained. Agitating the fiber suspension uniformly distributes the fibers in the suspension but creates large bubbles in the fluid. The act of sucking the fluid into a syringe removes these bubbles and maintains the uniform fiber distribution, illustrated in Figure 3.16, which is maintained while dispensing the fiber suspension into the mold and sensing apparatus. Finally, the length of time current can be applied to the solenoid is dependent on the magnitude of the applied current, presented in Table 3.3. 


\begin{tabular}{|c|c|c|c|}
\hline Applied current (A) & Time (s) & Applied current (A) & Time (s) \\
\hline 5 & 90 & 25 & 45 \\
10 & 75 & 30 & 30 \\
15 & 60 & 35 & 30 \\
20 & 45 & 40 & 15 \\
\hline
\end{tabular}

Table 3.3: The time current was applied to the solenoid was dependent on the magnitude of the current in order to mitigate thermal effects.

The accurate measurement of fiber rotation in the solenoid requires an even fiber distribution and no air bubbles in the mold. Additionally, the conductance of the unaligned fiber suspension is required to observe the change of conductance due to gravitational effects. Finally, the length of time current can be applied to the solenoid is dependent on the magnitude of the applied current, presented in Table 3.3. A set of process steps were devised as follows:

- The bulk fiber suspension is agitated to crate a uniform fiber distribution in the glycerol-water solution.

- $20 \mathrm{ml}$ of the fiber suspension is injected into the mold.

- The conductivity of the solution at rest is measured for 30 seconds at half second intervals.

- Current is applied to the solenoid, producing a magnetic field whist continuously measuring the conductivity of the solution.

- The current is removed from the solenoid while measuring the conductivity for 30 seconds.

In order to accurately measure the fiber rotation and isolate effects introduced by heating and the glycerol-water solution, a control experiment was conducted with a range of glycerol-water solutions that do not contain fibers. Fibers were then added to the solutions following the same procedure. This 
experiment requires a high viscosity solution as vertical drift in the fibers could change the fiber density in the sensing region effecting the conductivity.

The magnetically assisted fiber settling is very similar to the fiber rotation procedure. The experiment is robust in that the fiber distribution in the suspension has minimal impact on the resulting clearing time, except in extreme cases where all fibers are injected into the bottom portion of the mold and none are in the top. However for consistency a uniform fiber distribution is created in the solution.

- The bulk fiber suspension is agitated to crate a uniform fiber distribution in the glycerol-water solution.

- $12 \mathrm{ml}$ of the suspension is injected into the mold.

- The conductivity of the empty cell is measured to ensure the motion of fibers immediately after injection is captured.

- The conductivity of the solution is measured using an electrode pair selected through selecting a specific output of the multiplexor.

- The multiplexor output is changed to the next electrode pair and the conductivity is measured. This is repeated until all electrode pairs have been selected by the multiplexor. Electrode pairs are illustrated in figure $3.9 \mathrm{~b}$

- Repeat the measuring of electrode pairs until the conductivity across all pairs becomes constant, indicating there is no more fiber movement in the suspension.

\subsubsection{Optical Experiments}

The optical monitoring of fiber motion is achieved through video analysis. Optical access to the fibers is provided through the use of a Helmholtz coil and an open magnetic cell. The motion of fibers are captured using a Basler camera. 
The experimental process for these experiments are identical aside from the requirement to apply current to the Helmholtz coil. The experimental procedure is as follows:

- The bulk fiber suspension is agitated to crate a uniform fiber distribution in the glycerol-water solution.

- The camera begins recording.

- The mold is filled with the fiber suspension.

- For fiber rotation experiments, current is applied to the Helmholtz coil until fiber motion ceases. 
CHAPTER 3. EXPERIMENTAL METHODOLOGIES 


\section{Chapter 4}

\section{Fiber Rotation}

The rotation of the fiber in a magnetic field is a key component of the creation of porous piezoceramics. The degree of alignment between fibers dictates how uniformly the piezoceramic expands and contracts with an applied voltage; a property which limits the performance and resolution of the peizoceramic. Hence it is crucial to ensure an aligned array of fibers is produced during the piezoceramic manufacturing process.

Transmission optical microscopy is a technique commonly utilised in fiber rotation applications [13] [14] however PZT, the material used to manufacture piezoceramics, is opaque. This means non-optical techniques are required for the measurement of the fiber rotation. Furthermore, models for the rotation of these fibers assume either the fibers to be neutrally buoyant and a solid cylinder of one material. These conditions are not suitable for the materials used in the manufacturing of porous piezoceramics. Due to these factors, a non-optical technique has been developed whereby an electrode pair monitors the conductance of fibers suspended in an opaque solution over time. This change in conductance is compared to optically obtained fiber rotation using established microscopy techniques.

The time constant of rotation, defined as the time it takes for the fiber to complete $63 \%$ of its rotation, is used as a metric for comparing rotation times measure using these techniques described. This is due to the time constant be- 


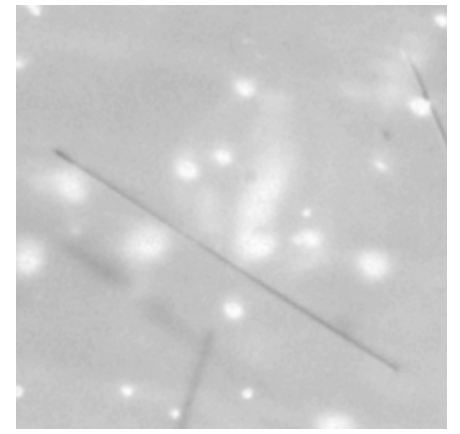

(a) 0 Seconds

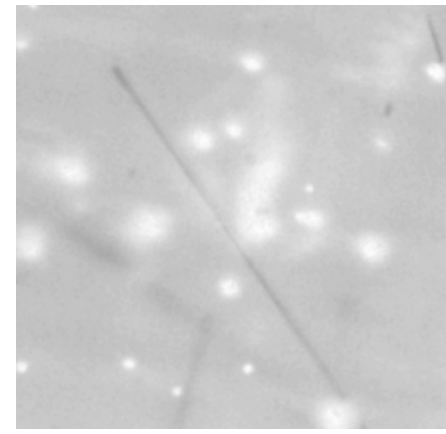

(b) 0.68 Seconds

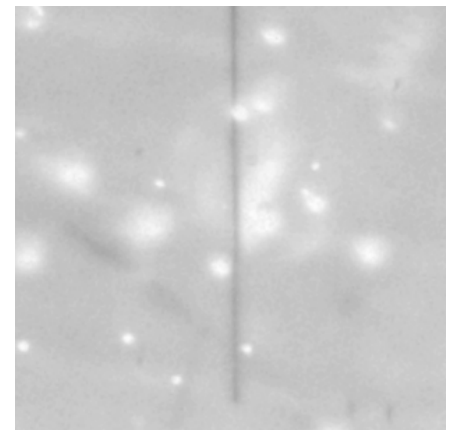

(c) 0.91 Seconds

Figure 4.1: The fiber rotation can be visually observed using a close focus camera in conjunction with a Helmholtz coil. The fiber starts randomly oriented (a) and with the application of a magnetic field, it rotates (b) until it is aligned with the magnetic field (c).

ing the main characteristic of first-order linear time invariant systems.

This chapter presents the results of optical measurement of fiber rotation in a transparent solution using an applied field form a Helmholtz coil. Numerical models are then compared to the optical measurements in order to model the motion of a single nickel coated carbon fiber. The model is extended for Monte Carlo simulations of single fibers to model a system of randomly oriented fibers. Finally, the fiber rotation measured though the electrodes are then compared to the Monte Carlo simulations in order to validate the electrode designs.

\subsection{Helmholtz coil}

Transmission optical microscopy is an optical technique whereby the object to be observed is backlit, and observed typically using a CCD camera. This technique used in conjunction with a Helmholtz coil can be used to measure the rotation of a single fiber subject to a magnetic field. An individual fibers rotation is illustrated in Figure 4.1, where the fiber is observed to rotate from an initial angle of 40 degrees until it is aligned vertically with the applied field.

In order to analyse the time constant of rotation, frame by frame analysis 
was conducted using 'Tracker', an open source motion tracking software [112]. Capturing the fiber rotation has an inherent limitation in that only the motion in the $2 \mathrm{D}$ viewing plane can be observed. This can be somewhat mitigated through the use of user defined 'points of interest' which are analysed through the Tracker software. In our case these are defined as the ends of a fiber. The software returns the location of the position of each point as an $(x, y)$ coordinate pair for each frame of the input video. This allowed for both the change in both the fiber angle relative to the $2 \mathrm{D}$ viewing plane and the observed length of the fiber to be measured. This change in fiber length gives the out-of-plane rotation, and hence the fiber angle relative to the magnetic field can be calculated. Furthermore, having multiple fibers in the recorded frame allows the ability to measure the lengths of the fibers and fit the length distribution to the fiber length distribution presented in Section 3.1. We can then approximate the length of the fibers using these distributions.

That said, it is not appropriate to analyse all fibers in the frame due to external interactions and anomalous effects resulting from this measurement technique. Fibers which are not observed to move over the duration of the experiment or pivot around one end of the fiber are considered to have become stuck or are otherwise interacting with the walls of the fluid container and should be ignored. Furthermore fragments of fibers with a length less than $50 \%$ of the bulk fiber lengths are rejected as they are considered anomalies and make up less than $2 \%$ of the overall fibers viewable using the Basler Camera.

The motion of a selection of fibers measured using this technique is illustrated in Figure 4.2. 0 degrees is horizontal in the recorded frame, whereas 90 degrees is vertical and hence parallel to the magnetic field. The fibers are observed to have a random initial angle at $t=0$. The application of a $30 \mathrm{mT}$ magnetic field results in the fibers rotating and aligning with the magnetic field. Both the viscosity and fiber length are observed to have a large impact on the fiber rotation time constant. A length difference between a fiber with a length of $0.4 \mathrm{~mm}$ and one $0.6 \mathrm{~mm}$ long corresponds to double the time constant of fiber rotation for fibers with similar initial orientation. 


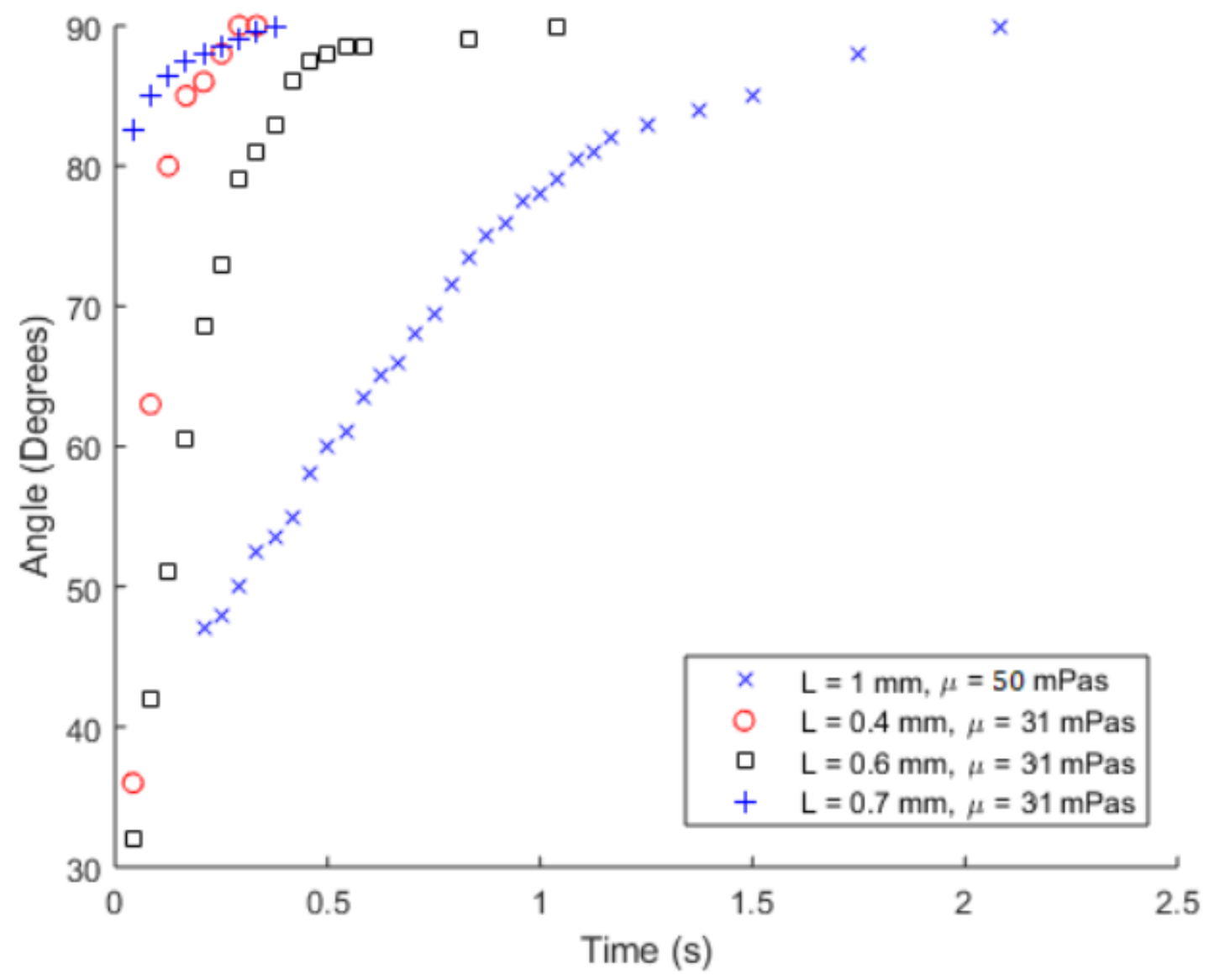

Figure 4.2: The rotation of fibers in a magnetic field generated via a Helmholtz coil and measured by a Basler camera shows a strong dependance on fiber length, fluid viscosity, and initial orientation. 


\begin{tabular}{c|c|c|c} 
Solution & Viscosity & Number of Fibers Analysed & Time Constant \\
\hline $85 \mathrm{wt} \%$ glycerol & $94 \pm 2 \mathrm{mPas}$ & 34 & 1.04 Seconds \\
$80 \mathrm{wt} \%$ glycerol & $51 \pm 2 \mathrm{mPas}$ & 43 & 0.95 Seconds \\
$75 \mathrm{wt} \%$ glycerol & $30 \pm 2 \mathrm{mPas}$ & 31 & 0.72 Seconds
\end{tabular}

Table 4.1: The time constant for fibers suspended in various glycerol-water solutions using a Helmholtz coil in conjunction with video analysis is presented. The fibers analysed were "complete" fibers with a length of $0.98 \pm 0.1 \mathrm{~mm}$, which constitute $87 \%$ of the bulk fibers.

This analysis was performed on a large number of fibers in varying fluid viscosities. The time constants are presented in Table 4.1. These time constants for fiber rotation are observed to be non-linear with fluid viscosity. This is inconsistent with literature, where the rotation of fibers in a Newtonian fluid is linear with viscosity [13] [68].

Fiber bending is expected to result in non-linear effects [72], however the fibers are measured to be rigid throughout the experiments. Instead, it appears the effective viscosity changes in the experiments. This change in effective viscosity is consistent with that of a thixotropic solution, where sheer thinning due to fibers rotating reduces the fluids viscosity. However, pure glycerol-water solutions are known to be Newtonian. This suggests the addition of fibers and their motion changes the effective viscosity of the solution. However, electrostatic forces between the fibers and the walls of the cell and magnetic dipole interactions may contribute to this effect. These viscosity effects are discussed in detail in Section 4.6.

\subsection{Mathematical Modeling of Fiber Rotation}

The rotation of magnetic fibers suspended in a fluid and subjected to a magnetic field is commonly modeled as a balance between the hydrodynamic and magnetic torque [68] [69] [70]. Shine et al. produced a simple model for the motion 
of long slender fiber in magnetic saturation, and the agreement between theoretical and experimental values of the rotation rate is noted to improve with high aspect ratio fibers. For these reasons, it will be used as a model for the rotation of fibers in a magnetic field. The model is described as

$$
\frac{4}{3}\left(\frac{L}{D}\right)^{2} n_{s}\left[\frac{\epsilon(1+0.64 \epsilon)}{1-0.5 \epsilon}+1.019 \epsilon\right]\left(-\Omega_{z}\right)+\frac{\mu_{0} H_{0}^{2} M_{s}^{2}\left(D_{y y}-D_{x x}\right) \sin \beta \cos \beta}{\left(H_{i}+D_{x x} M_{s}\right)\left(H_{i}+D_{y y} M_{s}\right)}=0
$$

where $L$ is the length of the fiber, $D$ is the diameter of the cylinder, $n_{s}$ is the viscosity of the fluid, $\mu_{0}$ is the magnetic permeability of free space given by $\mu_{0}=4 \pi \times 10^{-7} \mathrm{H} / \mathrm{m}, \beta$ is the fiber angle relative to $H_{0}$, and $M_{s}$ is the magnetic saturation magnitude experienced by the fiber. $\Omega_{z}$ is the angular velocity of the rotating fiber about the z-axis. $\epsilon$ is the small parameter of slender body theory [75] described as

$$
\epsilon=1 / \ln (2 L / D)
$$

$D_{x x}$ and $D_{y y}$ are volume averaged demagnetization factors for an ellipsoid equivalent to a uniform magnetized cylinder, and are described as

$$
\begin{gathered}
D_{x x}=\frac{4}{3 \pi}\left(\frac{D}{L}\right)-\frac{1}{8}\left(\frac{D}{L}\right)^{2}+\frac{1}{64}\left(\frac{D}{L}\right)^{4}-\frac{5}{512}\left(\frac{D}{L}\right)^{6}+\ldots, \\
D_{y y}=\left(1-D_{x x}\right) / 2 .
\end{gathered}
$$

$H_{0}$ is the externally applied magnetic field in $\mathrm{A} / \mathrm{m}$ and $H_{i}$ is the induced magnetic field inside the ferromagnetic body, described as

$$
H_{i}=H_{0}-D^{p}(r) \cdot M
$$

where $D^{p}(r)$ is the position dependent demagnetization tensor, $r$ is the position vector, and $M$ is the magnetization of the particle at each point.

The model does have some limitations in its applicability to our experiments. The time dependent demagnetisation tensor used holds for a solid ferromagnetic fiber, but is geometry dependent and hence the calculation used to 
obtain this value not applicable for a ferromagnetic tube. That said, it is an integral component of the model and hence a value for this parameter will be estimated based on literature values of comparable geometries [113].

\subsubsection{Single Fiber Modeling}

Using Equation 4.1, the motion of a single fiber in a Newtonian fluid suspension can be modeled as a function of time, fluid viscosity, and initial orientation. These conditions all effect the time constant of rotation, which is important for the manufacturing of piezoceramics and other materials with aligned microwires.

The motion of a single fiber as a function of initial angle is presented in Figure 4.3. The time constant of rotation is observed to reduce as the initial angle becomes closer to parallel with the magnetic field. This suggests flow alignment may be used to provide a favorable initial orientation for the fibers, which can be achieved through the injection of the fluid through a syringe or other tube which promotes laminar flow [114]. This effect may assist the piezoceramic alignment process during wafer fabrication. Low viscosity solutions reduce the hydrodynamic torque on the fibers. This results in a reduction in the time constant of fiber rotation, illustrated in in Figure 4.4.

A comparison between the fiber rotation measured using the Helmholtz coil and the model is presented in Figure 4.5. The model shows good accordance with the Helholtz experimental results when adjusted for fluid viscosity. The degree of adjustment required to accurately model the fiber rotation increases for higher fluid viscosities.

\subsubsection{Monte-Carlo Simulation}

A Monte Carlo simulation is a good model for bulk fiber motion where the fiber length and initial orientation has a large impact on the fiber motion. Generating a large number of fibers with a uniform random angle distribution and length distribution in accordance with that presented in Section 3.1 allows for 


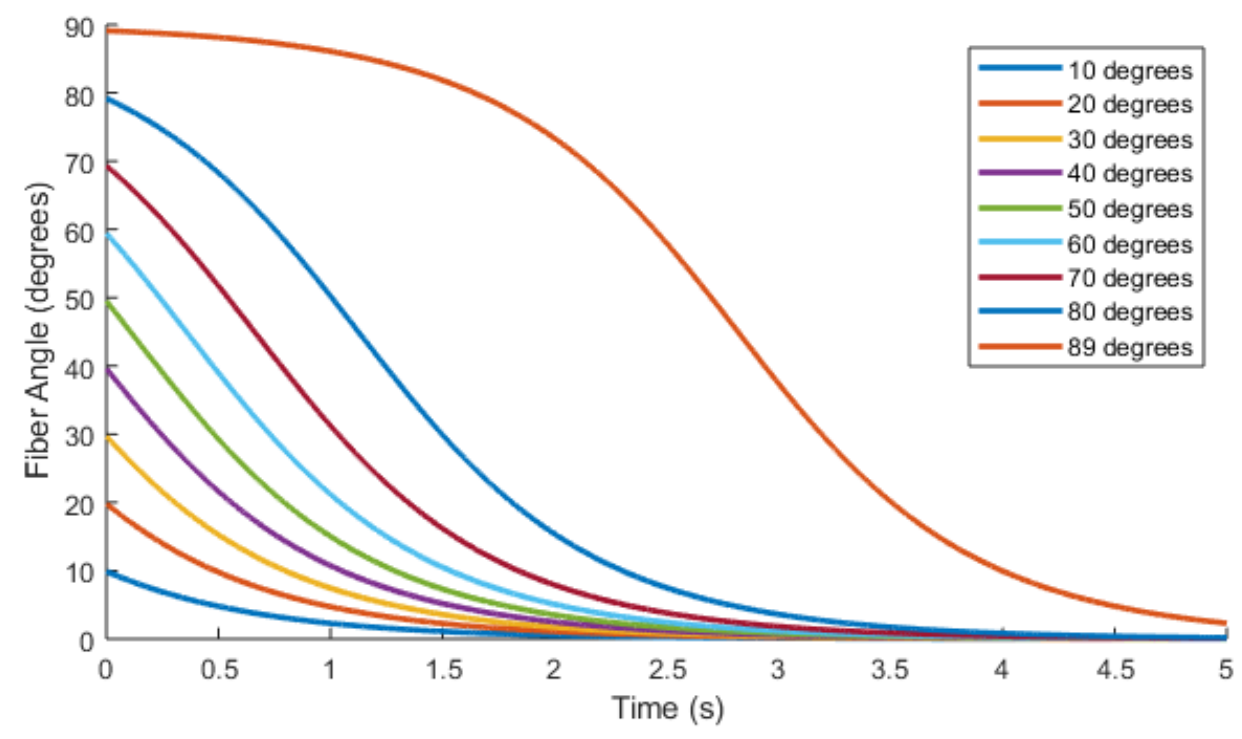

Figure 4.3: Balancing the hydrodynamic torque and magnetic torque for a single fiber in magnetic saturation using the equation described in Equation 4.1 results in the alignment of the fiber with the magnetic field with the time constant of rotation being a product of the initial fiber orientation. The fluid viscosity for this modeling is $100 \mathrm{mPas}$ with a field strength of $30 \mathrm{mT}$. In this model, 90 degrees denotes the fiber is perpendicular to the magnetic field, and 0 degrees is parallel to the magnetic field. The legend describes the initial orientation of the modeled fibers relative to the simulated magnetic field. 


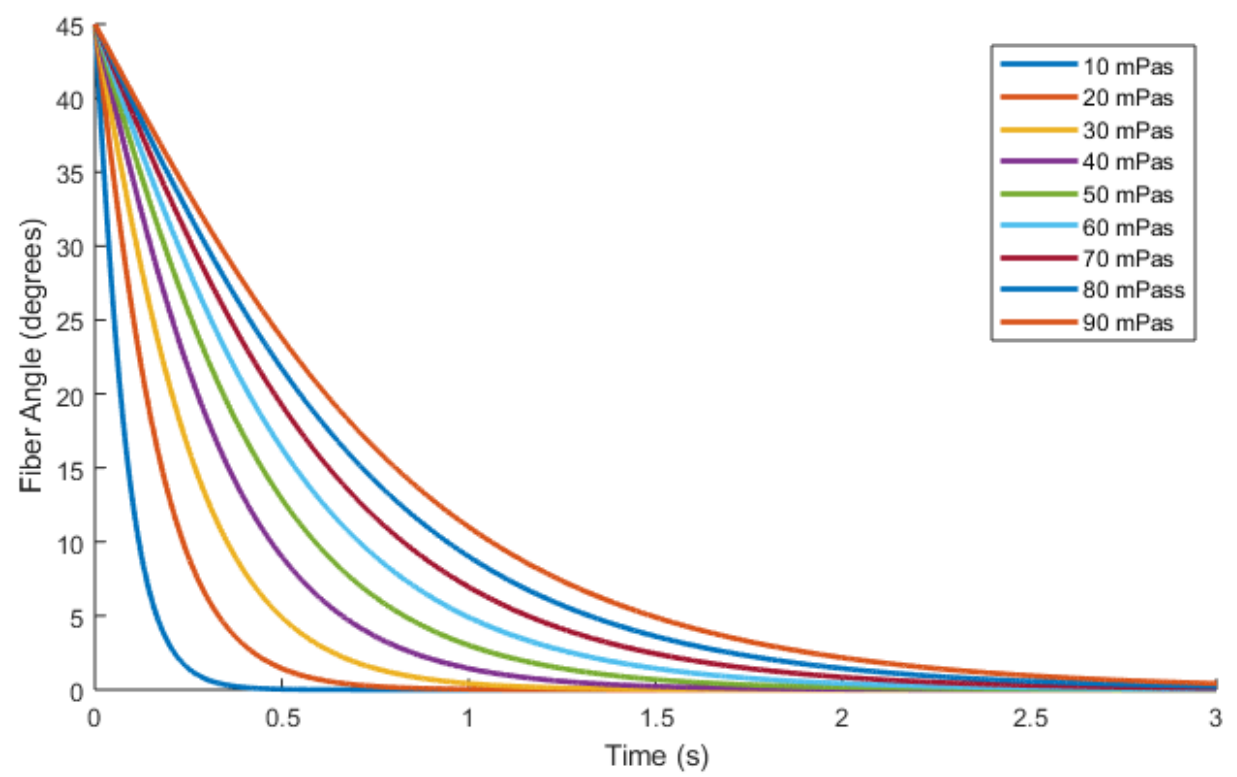

Figure 4.4: Increasing the modeled fluid viscosity results in a linear increase in the time constant of rotation for fibers suspended in a glycerol water solution. The initial angle in this modeling is fixed at 45 degrees with a field strength of 30 $\mathrm{mT}$. In this model, 90 degrees denotes the fiber is perpendicular to the magnetic field, and 0 degrees is parallel to the magnetic field. 


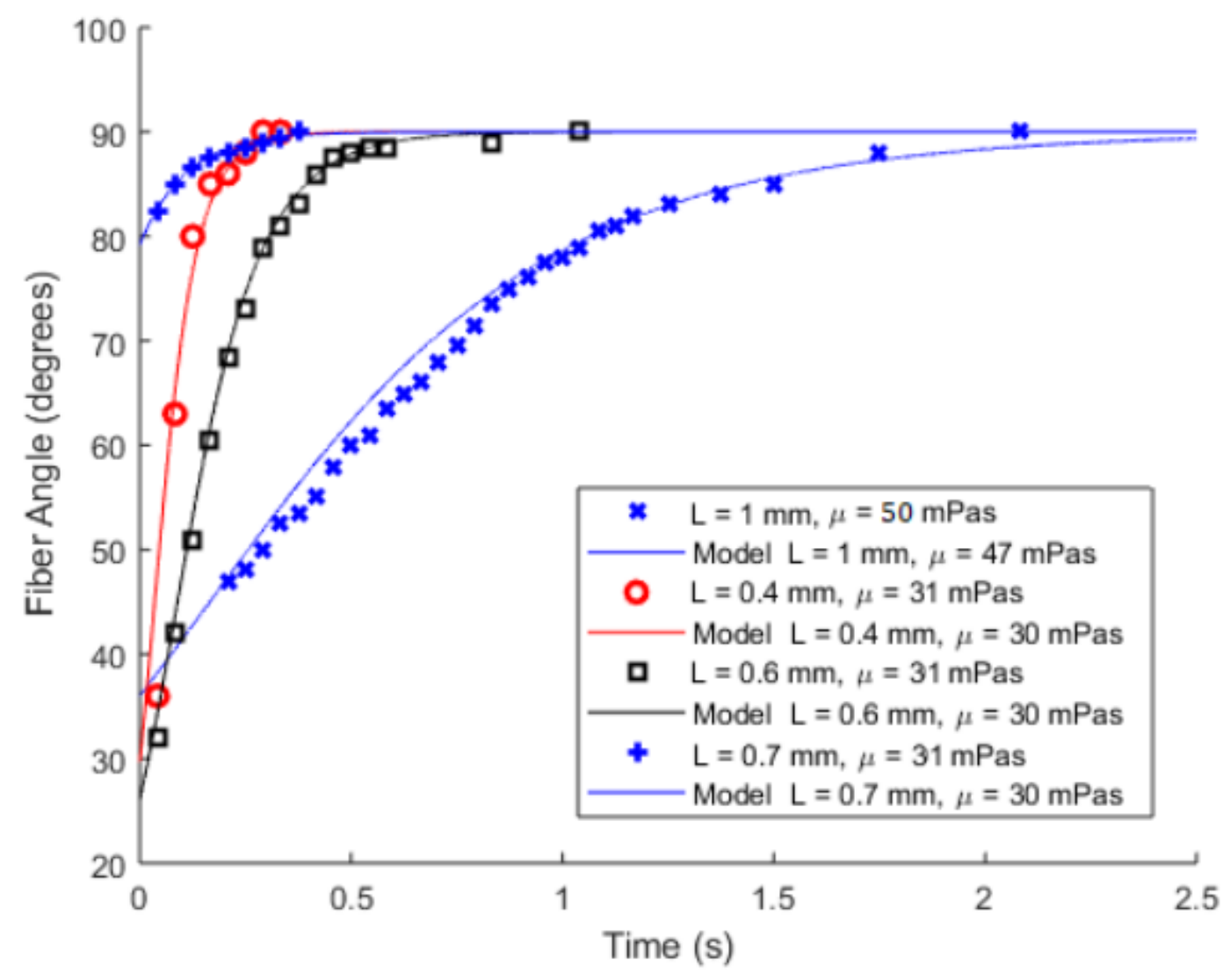

Figure 4.5: The analytical model shows good agreement with the observed fiber motion using the Helmholtz experiments after adjustments to the modeled fluid viscosity. 90 degrees denotes alignment with the magnetic field. 
a complete analysis of the fiber rotation measured using electrical experiments. The resulting average angle as a function of time is presented in Figure 4.6 for a number of fluid viscosities. The modeled average fiber angle is linear with viscosity.

\subsection{Results of Fiber Rotation using Electrical Tech- niques}

Non-optical techniques for measuring the rotation of fibers have not been explored in literature. In order to attempt to fill this knowledge gap, two electrode configurations have been fabricated in order to measure fiber rotation. These electrodes use the electrical properties of isotropic materials, where the alignment of fibers change the bulk materials conductance.

The alignment of the fibers requires the application of a uniform magnetic field. This was generated in the core of a solenoid. However the generation of this field requires passing current through the solenoid, which is known to generate heat and hence change the viscosity of the fluid suspension. Furthermore, premagnetisation of the fibers may occur in the manufacturing, storage, or processing of the fibers. Therefore it is prudent to consider these effects on the bulk conductance before validating the electrode configurations and measurement system.

\subsubsection{Heating in the solenoid}

The application of current to a solenoid results in the generation of heat due to the resistive nature of the coil. This creates a region of heated air in the core of the solenoid. The glycerol-water solution is heated up by this hot air, effecting the viscosity and conductivity of the fluid. This effect is illustrated in Figure 4.7, where the application of a 42 A current to the solenoid denoted by the red portion results in a drastic change in the conductivity of the glycerolwater solution. However, this change in temperature has a long time constant, 


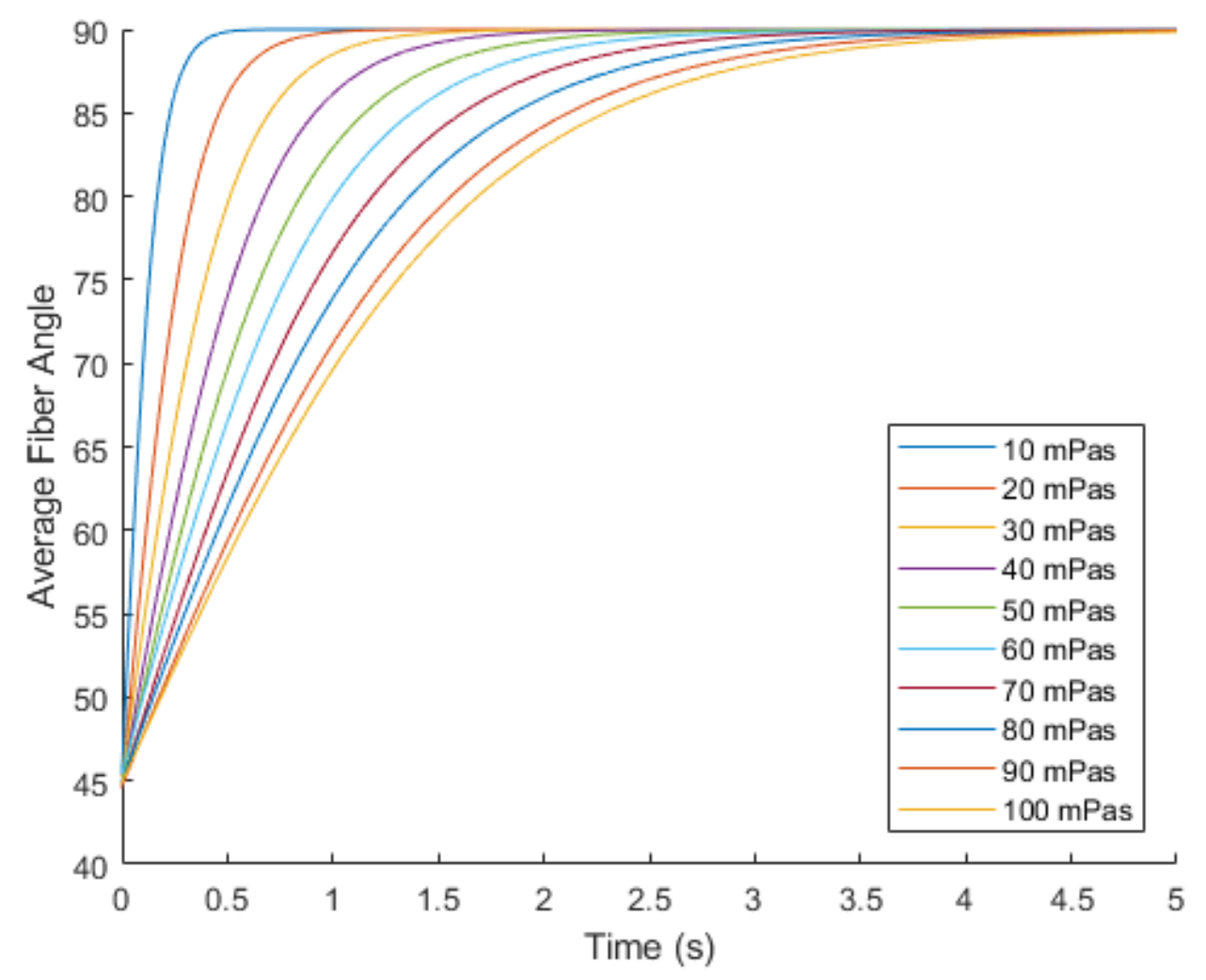

Figure 4.6: Using a Monte Carlo simulation we can model a large number of fibers with a uniform-random initial orientation relative to the magnetic field, and a fiber length distribution which matches the measured lengths. This produces the average fiber angle as a function of time for a number of fluid viscosities. 
much longer that that of fiber rotation. The change in temperature over the period of fiber rotation was measured using a thermocouple, and was found to be $0.1 \pm 0.01^{\circ} \mathrm{C}$. This results in a maximum change of $1 \mathrm{mPas}$ for the glycerol-water solutions used.

Practical techniques can be implemented to thermally isolate or mitigate the fluid from the effects of current induced heating in the solenoid. Water cooling is one such example, whereby a tube containing running water is placed between the solenoid and the glycerol-water solution. Creating this additional component was determined to be excessive, as the thermal effects over the period of interest were observed to have a minimal change in viscosity. A simple method for displacing the warm air was implemented using a small desk fan. The desk fan was located approximately $1 \mathrm{~m}$ away from the experimental apparatus as to avoid any magnetic field superposition due to the fans motor. Although the fan displaced a potion of the warm air, heating effects were not completely removed.

Heating effects from the solenoid can be manipulated to compensate for differences in the ambient temperature during the experimental process. This allows for greater consistency in the viscosity and conductivity of the glycerolwater solutions as ambient temperatures are known to fluctuate between 18 and $23^{\circ} \mathrm{C}$ corresponding to $50 \mathrm{mPas}$ fluctuations in $85 \mathrm{wt} \%$ glycerol-water solutions.

To incorporate the current induced heating in the solenoid to maintain a stable temperature, a current is applied to the solenoid after the injection of the fiber suspension in the mold. This current is maintained until the fluid suspension reaches a desired temperature. The current is then removed and the fibers are manually agitated using a combination of shaking and stirring to remove any alignment the field may have induced. After agitation, the process outlined in Section 3.5.2 is performed. This heating process requires currents greater than the magnetic coercivity of the fibers, which leaves the fibers with remnant magnetisation. This premagnetisation is discussed in Section 4.3.2.

Minor fluctuations in temperature may still be present due to cooling while the baseline measurements are taken. The measurable effects of this tempera- 


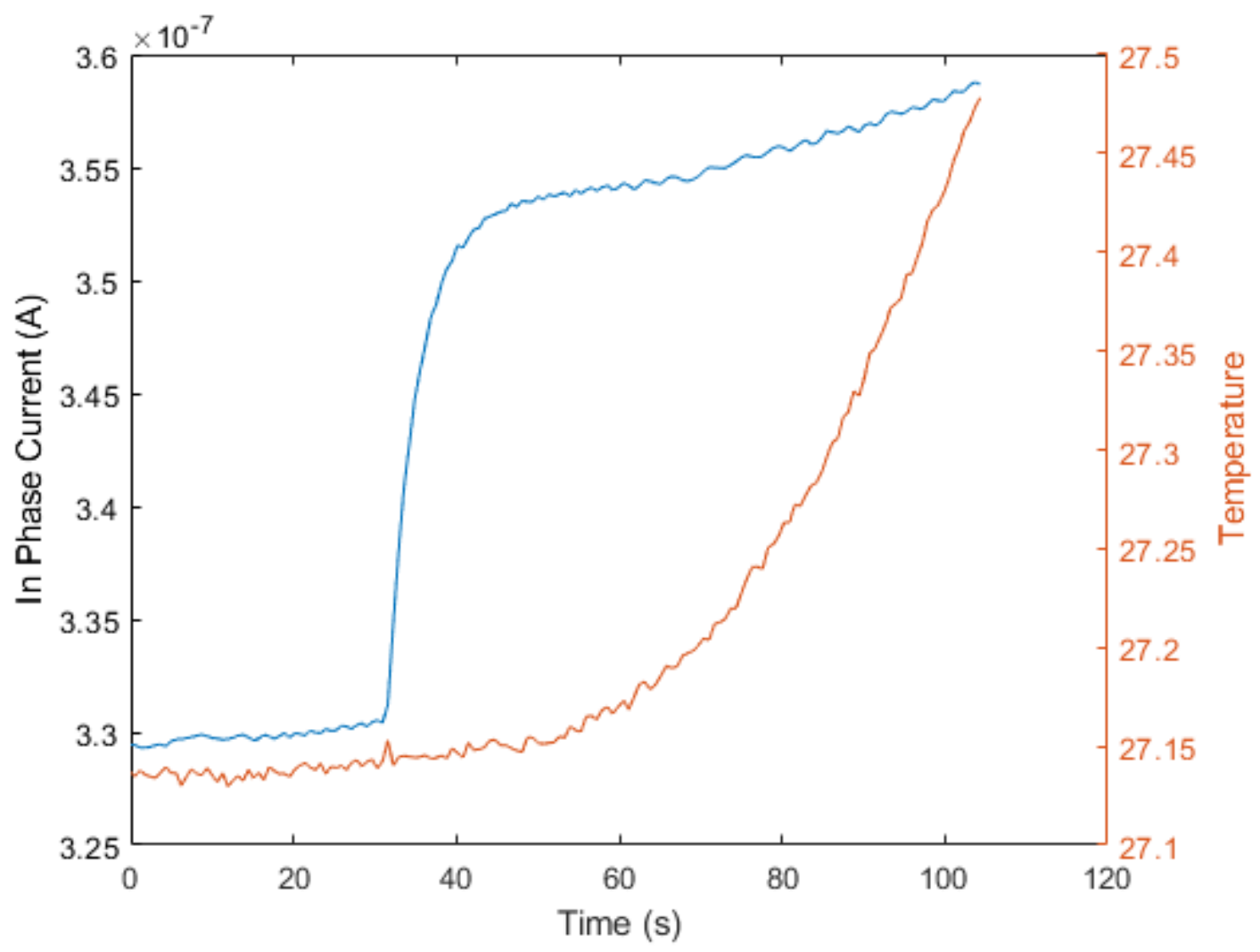

Figure 4.7: The time constant of fiber rotation is observed to be much less than the time constant of heating in the solenoid. An increase of $0.05^{\circ} \mathrm{C}$ is observed during the fiber rotation results in a viscosity decrease of $0.11 \mathrm{mPas}$. 


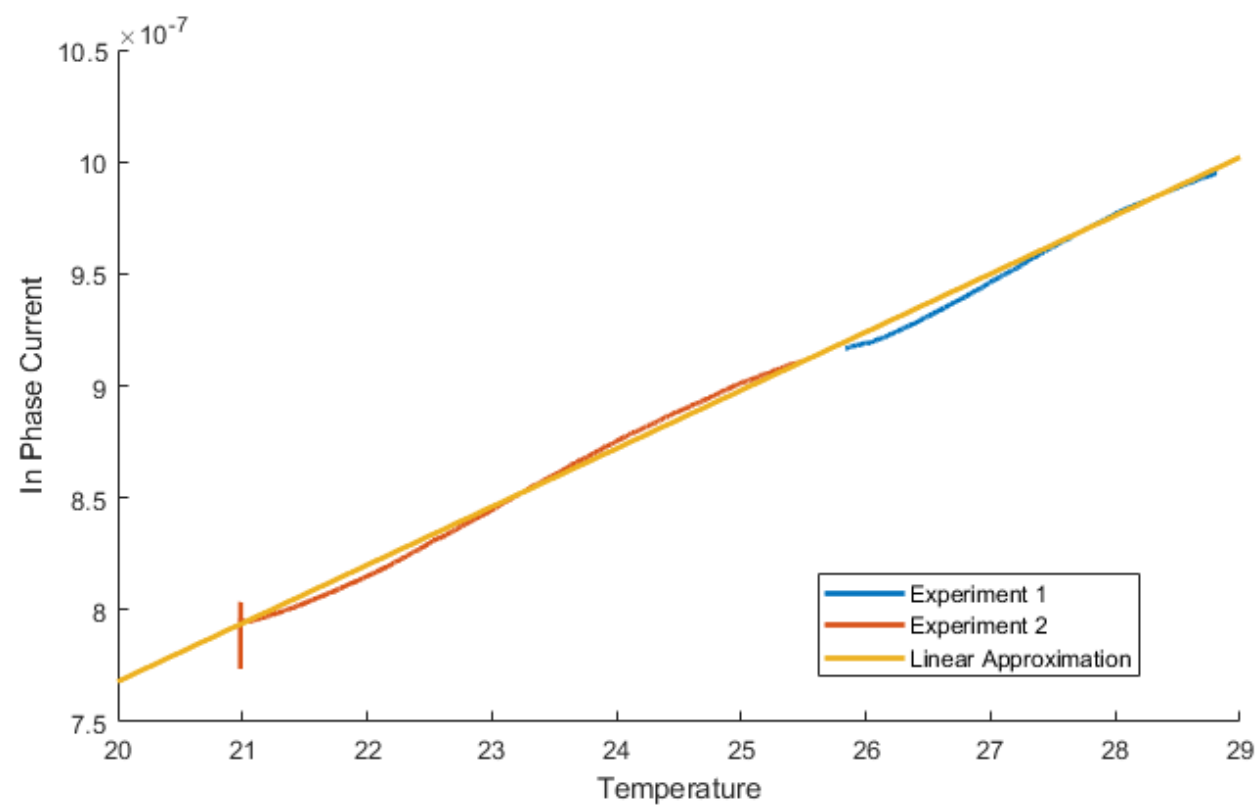

Figure 4.8: The conductance of 75 weight percent glycerol water solutions was heated using the solenoid experimental setup, and was found to increase approximately linearly with temperature.

ture change can be removed through the characterisation of the fiberless fluid suspension with temperature. This is illustrated in Figure 4.8 where the conductivity of a $75 \mathrm{wt} \%$ glycerol-water solution has been characterised across an $8^{\circ} \mathrm{C}$ temperature range.

The conductance of the fiber-fluid suspension can be modeled as the fiber conductance and the fluid conductance. The fiber conductance is a function of the number density of fibers per unit volume, the orientation of these fibers, and the temperature. The conductivity of the fluid is simply a function of the temperature and fluid composition. This can be expressed generally as

$$
G_{t}=G(g, T)+G(n f, T) \pm G(O, H),
$$

where $G_{t}$ is the total measured conductivity of a fluid suspension containing fibers, $G(g, T)$ is the conductivity of fluid composition without fibers, $G(n f, T)$ is the conductivity contribution from the number density of fibers, and $G(O, H)$ 
is the change in conductance due to the orientation of fibers. This decomposition approximates the complex system of fluid and fibers as a basic parallel network, and results in the ability to subtract the effects of the glycerol-water solution from the conductance measured by the lock-in amplifier. The result of this is illustrated in Figure 4.9 where the conductivity of $75 \mathrm{wt} \%$ glycerol-water is removed from the in-phase current of a glycerol-water solution containing 4 fibers $/ \mathrm{mm}^{3}$. The initial conductivity before the application of the magnetic field is not observed to be constant. This is a product of both a change in temperature and the gravitational forces acting on the fibers. Through the removal of temperature effects from the in-phase current, we are left with a change in the fiber concentration in the sensing region due to gravitational settling. The in-phase current after the temperature effect removal is observed to be negative. This is due to the addition of the nickel coated carbon fibers to the glycerol-water solution, and the simplification of a complex system to the basic parallel network described in Equation 4.6.

The magnitude of the change in conductance due to the alignment of the fiber was measured to be a function of the applied field strength. For a large applied field sufficient to magnetically saturate the fibers, the change in conductance is observed to be less than that of a smaller applied field which does not put the fibers in saturation. This is presented in Figure 4.10, where the change in conductivity due to the application of a magnetic field is presented for a number of field strengths, number densities of fibers and fluid compositions.

A temperature increase in the fluid resulting in an increase in the fluids conductance was an idea explored to explain this phenomenon. The temperature increase observed during experiments is on average less than $0.01{ }^{\circ} \mathrm{C}$ which has a change in conductance of less than $0.002 \times 10^{-8} \mathrm{~A} / \mathrm{V}$, much less than the observed change with field strength. This effect is most likely due to a change in the nickel resistance due to the magnetisation [115] [116]. In any case, it is not expected to be a result of the degree of alignment in the fibers as a larger change in conductance corresponds to a greater degree of alignment, which we would expect from applied fields with greater magnitudes. Hence this effect, while 

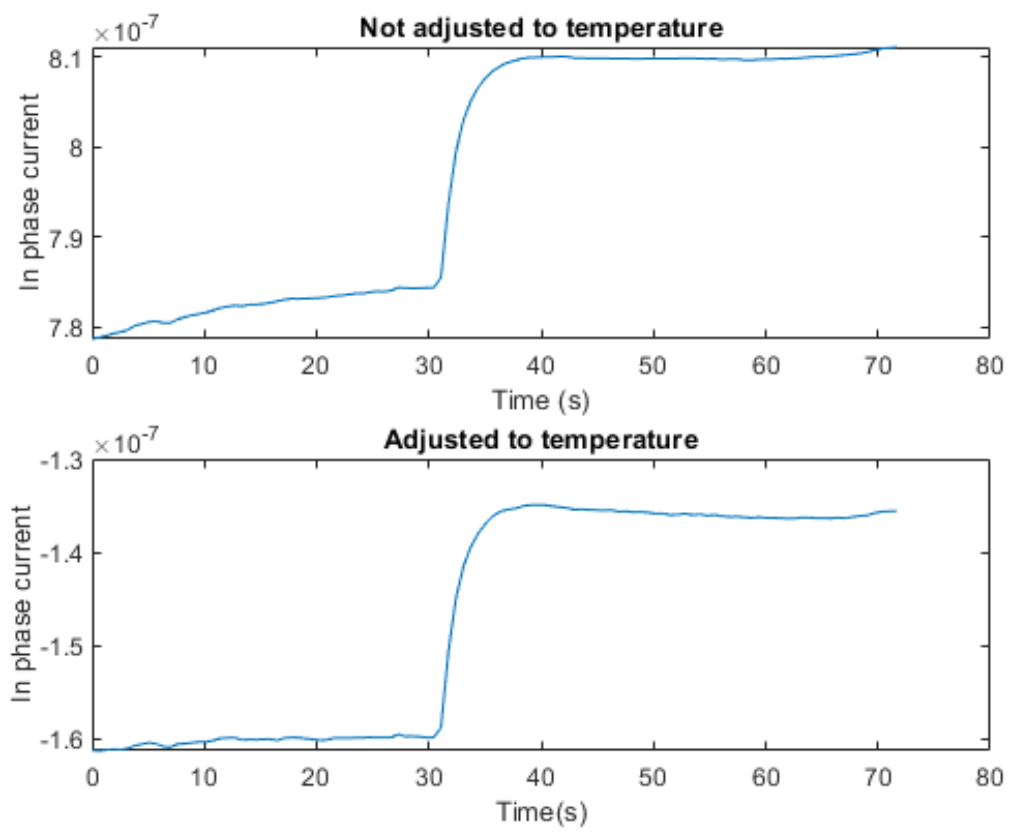

Figure 4.9: The application of a temperature adjustment algorithm allows for temperature controlled viscosity manipulation in the solenoid though isolating the effects of changing temperature from the application of current though the solenoid. The applied current in this example is $20 \mathrm{~A}$ to a 75 weight percent glycerol water solution containing 4 fibers per cubic millimeter. 


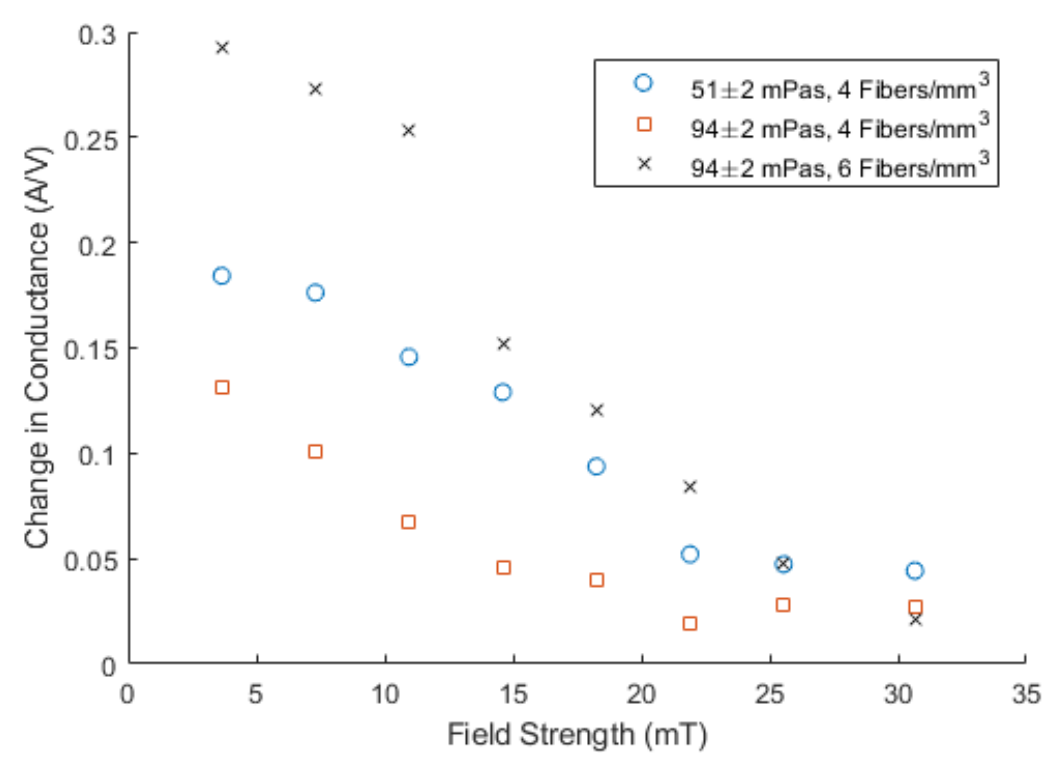

Figure 4.10: The change in conductivity between randomly oriented and aligned fibers is observed to be a function of the applied field strength and the number density of the fibers. The fluid composition does not effect this conductivity change.

interesting, is not crucial to the manufacturing of porous piezoceramic wafers.

\section{Magnetic Hysteresis}

In order to analyse the effects of the fibers magnetic hysteresis on the fiber rotation, the same fibers were used for 3 consecutive experiments. The first and third experiments had a small applied field (14.6-26.2 $\mathrm{mT})$ in the solenoid, and the second had a comparatively strong magnetic field $(30.66 \mathrm{mT})$. The glycerolwater solution was agitated with wooden stick to randomly orient the fibers before each experiment. The results are presented in Table 4.2. A comparison between the time constant of the first and third experiment shows for a range of fluid viscosities there is a small increase in the time constant. This is due to the alignment of magnetic domains preceding the magnetic torque which causes fiber rotation. Larger applied magnetic fields are observed to reduce the change 


\begin{tabular}{c|c|c|c|c}
$\begin{array}{c}\text { Fluid } \\
\text { Viscosity }\end{array}$ & $\begin{array}{c}\text { Field Strength } \\
1 \text { and 3 }\end{array}$ & $\begin{array}{c}\text { Field } \\
\text { Strength 2 }\end{array}$ & $\begin{array}{c}\text { Time } \\
\text { Constant 1 }\end{array}$ & $\begin{array}{c}\text { Time } \\
\text { Constant 3 }\end{array}$ \\
\hline $31 \pm 2$ & 26.2 & 30.66 & 1.64 & 1.75 \\
$31 \pm 2$ & 14.6 & 30.66 & 3.20 & 3.31 \\
$51 \pm 2$ & 26.2 & 30.66 & 1.76 & 1.83 \\
$51 \pm 2$ & 14.6 & 30.66 & 3.49 & 3.62 \\
$94 \pm 2$ & 26.2 & 30.66 & 1.94 & 2.01 \\
$94 \pm 2$ & 14.6 & 30.66 & 4.03 & 4.21
\end{tabular}

Table 4.2: The effects of remnant magnetisation in the fibers results in a small change in the time constant of rotation.

in time constant compared to smaller magnetic fields. A maximum difference of $0.18 \mathrm{~s}$ is observed for high viscosity solutions which is deemed to be significant. Therefore fibers subject to magnetic fields which put the fibers in magnetic saturation are discarded after a single experiment.

\subsubsection{Fiber Relaxation}

The alignment of fibers is suggested through a change in conductance across the electrodes as a result of the application of a magnetic field. Upon the removal of the magnetic field, the fibers should lose their alignment as the gravitational settling effects dominate the fiber motion. The orientation of slender bodies in still water [117] suggest a perfect cylindrical body with a center of mass located at the center of volume will orient itself horizontally in free fall. Figure 4.11 presents the change in conductance as observed using the ring electrode configuration suggests the removal of the magnetic field results in partial randomisation in the fiber angle. This is consistent with slender bodies in free fall where the center of mass is offset from the center of volume [118] which may be the case for damaged fibers, however it could also be a result of the interaction between the remnant magnetisation of a number of spatially dense fibers, whereby free motion is limited due to the balance of remnant magnetism 


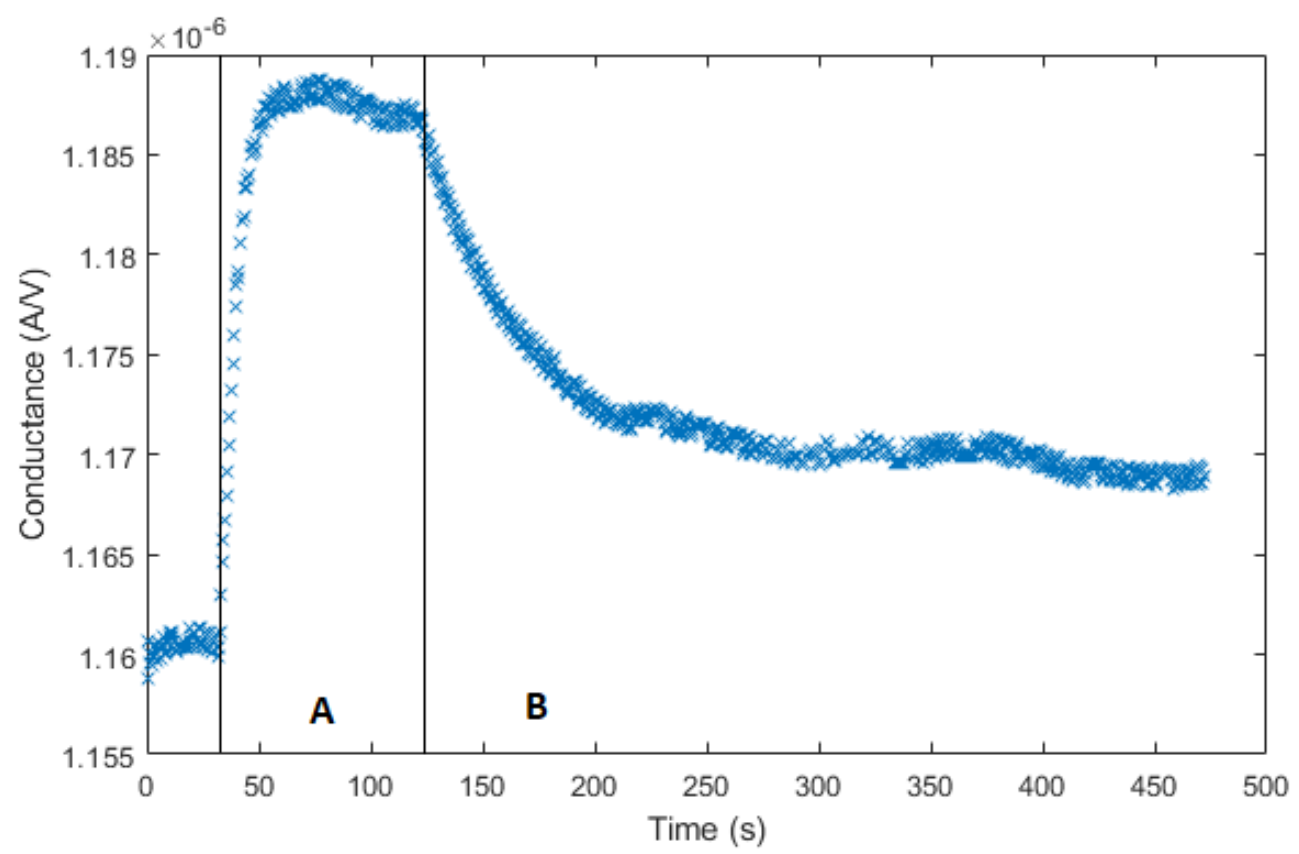

Figure 4.11: The increase in conductance with the application of a magnetic field (region A) is indicative of the fiber rotation in the solenoid. Upon removing the magnetic field (region B) the conductance decays to a constant level greater than the initial conductance, indicating the fibers remain partially unaligned.

and gravitational effects [119]. In any case, for piezoceramic fabrication applications this observed effect suggests the magnetic field should be applied until the ceramic has hardened.

\subsection{Quadrant Electrode Configuration}

The quadrant electrode configuration described in Section 4.4 is designed to measure a reduction in conductance for aligned fibers compared to unaligned fibers. This is because the applied magnetic field is orthogonal to the electric field produced by the electrodes. The recorded measured change in conductance is presented in Figure 4.12, where the conductance exhibits a clear transition from a stable conductance to a global minimum, followed by heating effects 


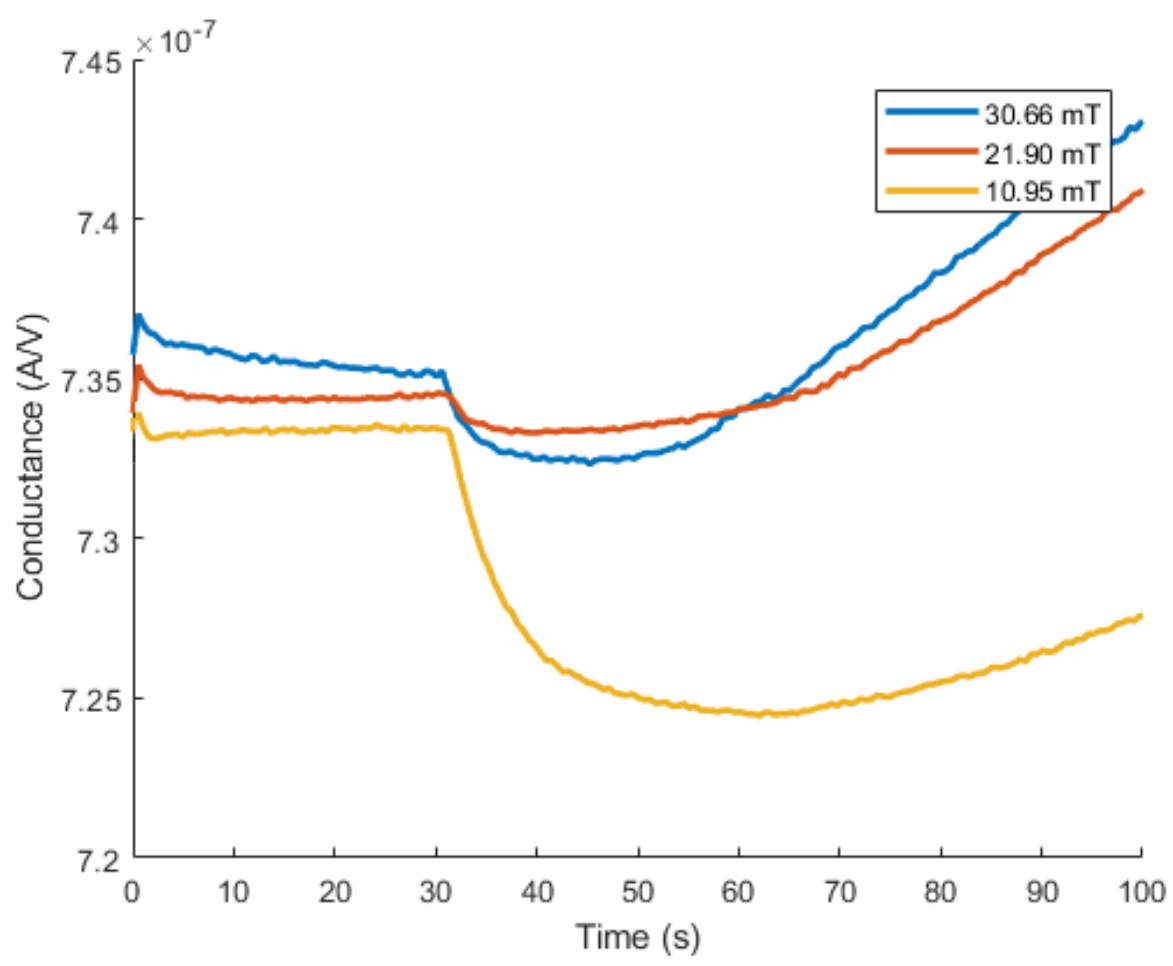

Figure 4.12: The application of a magnetic field at 30s results in a reduction in conductance while the fibers are rotating. The conductivity becomes constant once the fiber are aligned, and increases once the solenoid induced heating reduces the suspensions resistance. In this example, a 94 mPas solution containing 4 fibers per cubic millimeter was used.

increasing the conductance over time. From this raw data, the temperature effects can be removed as described in Section 4.3.1, and a time constant for the change in conductance can be obtained. Due to the geometry of the electrode configuration and the volume of fibers in the electrode sensing region, the fiber angles can not be directly obtained from this conductance change. However, comparisons can be made between the conductance time constant and the optically obtained time constant of rotation for fibers suspended in an identical fluid. This comparison is made in Section 4.6.

The time constants of the change in conductance for a range of fiber concentrations are presented in Figure 4.13. For the application of magnetic fields less 
than the saturation field strength, an increase in fiber concentration correlates to an increase in conductivity time constant. Increasing the field strength sufficiently to put the fibers in magnetic saturation results in the time constant of conductivity reducing such that it becomes approximately independent of fiber density. This suggests either fibers are weakly interacting with each other magnetically creating a lower net magnetic force on the fibers, or spatially dense fibers rotating creates additional turbulence in the suspension fluid resulting in additional hydrodynamic torque resisting the magnetically driven rotation for the bulk of the fibers. If the latter occurs, it would follow the effective viscosity of the fluid increases with fiber concentration.

An increase in the fluid viscosity is observed to result in an increase the conductance time constant, illustrated in Figure 4.14. However, this conductance time constant is non-linear with viscosity, as predicted by numerical modeling. This further strengthens the argument the effective viscosity is dependent on fiber concentration.

\subsection{Ring Electrode Configuration}

Similar to the quadrant electrode configuration, the ring electrode configuration measures bulk fiber orientation as a product of conductivity. The ring electrode has a more complex geometry, whereby the conduction path is hemispherical from one electrode to another. Furthermore the application of current from the lock-in amplifier produces a small inhomogeneous magnetic field parallel to the magnetic field produced by the solenoid. The raw change in conductance measured by the ring electrode configuration is illustrated in Figure 4.15. The conductance is observed to transition from a stable state to a local maximum. In this example, the heating effects and the glycerol-water solution component of the in-phase current are removed.

The time constant of conductance is observed to be independent of the fiber concentration when measured using the ring electrode, illustrated in Figure 4.16. This may be attributed to the superposition of the solenoid magnetic 


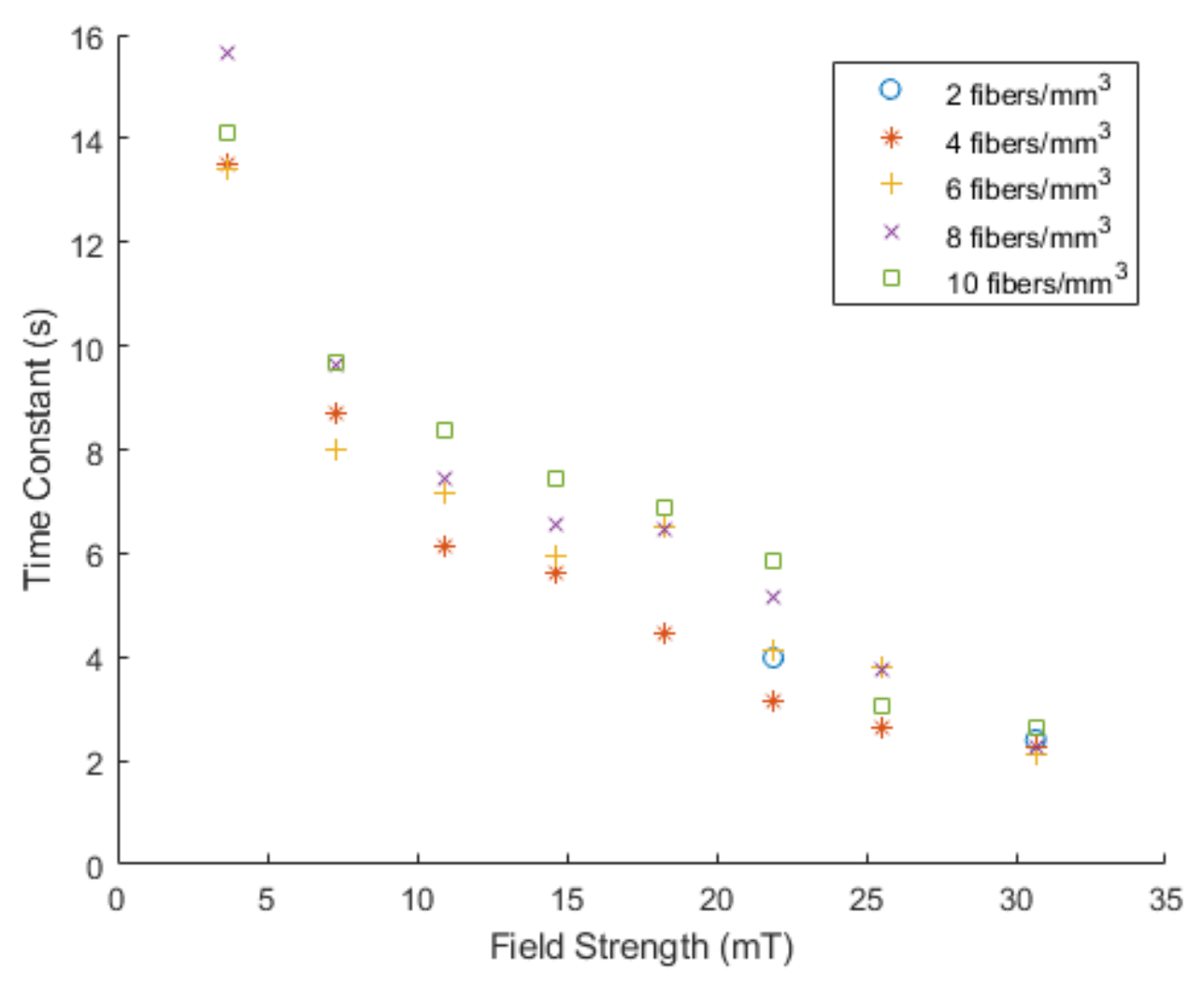

Figure 4.13: The time constant of rotation in the quadrant electrode configuration is observed to increase with fiber concentration when subject to low magnetic fields. The application of field strengths sufficient to put the fibers in magnetic saturation results in the time constant becoming independent of fiber concentration. 


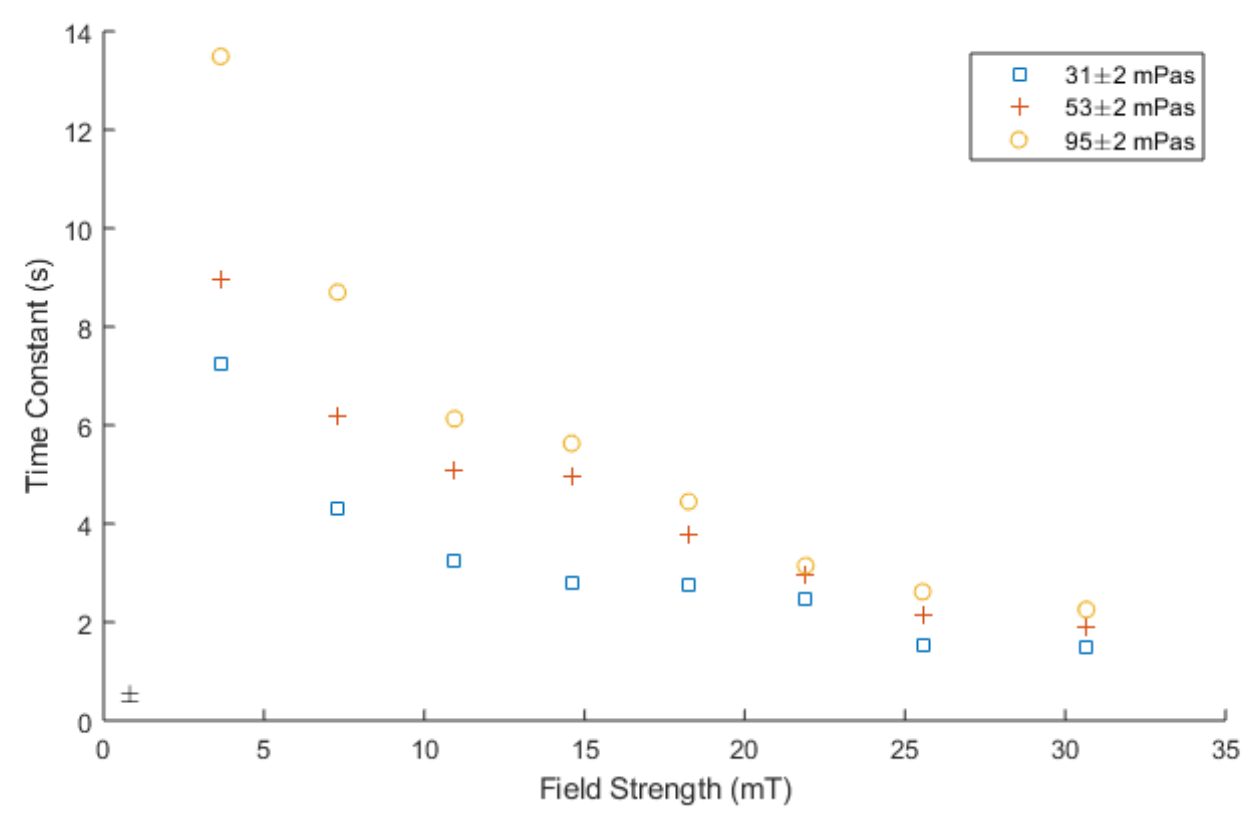

Figure 4.14: An increase in fluid viscosity is observed to increase the time constant of the change in conductance.

field and the AC field from the electrodes themselves. The conductance time constant approaches a limit at field greater than $20 \mathrm{mT}$, which is less than the field strength required for magnetic saturation, further strengthening the idea that the magnetic fields are superimposed.

Increasing the fluid viscosity is observed to result in an increase in the conductance time constant for applied fields grater than the coercive field strength, illustrated in Figure 4.17. The increase in conductivity is non-linear with viscosity, which is consistent with optical measurements.

\subsection{Discussion}

Transmission optical microscopy performed using a Helmholtz coil and CCD camera coupled with the conductance time constant results measured via the electrode configurations suggest the glycerol-water solution exhibits nonNewtonian characteristics with the application of fibers to the suspension fluid. 


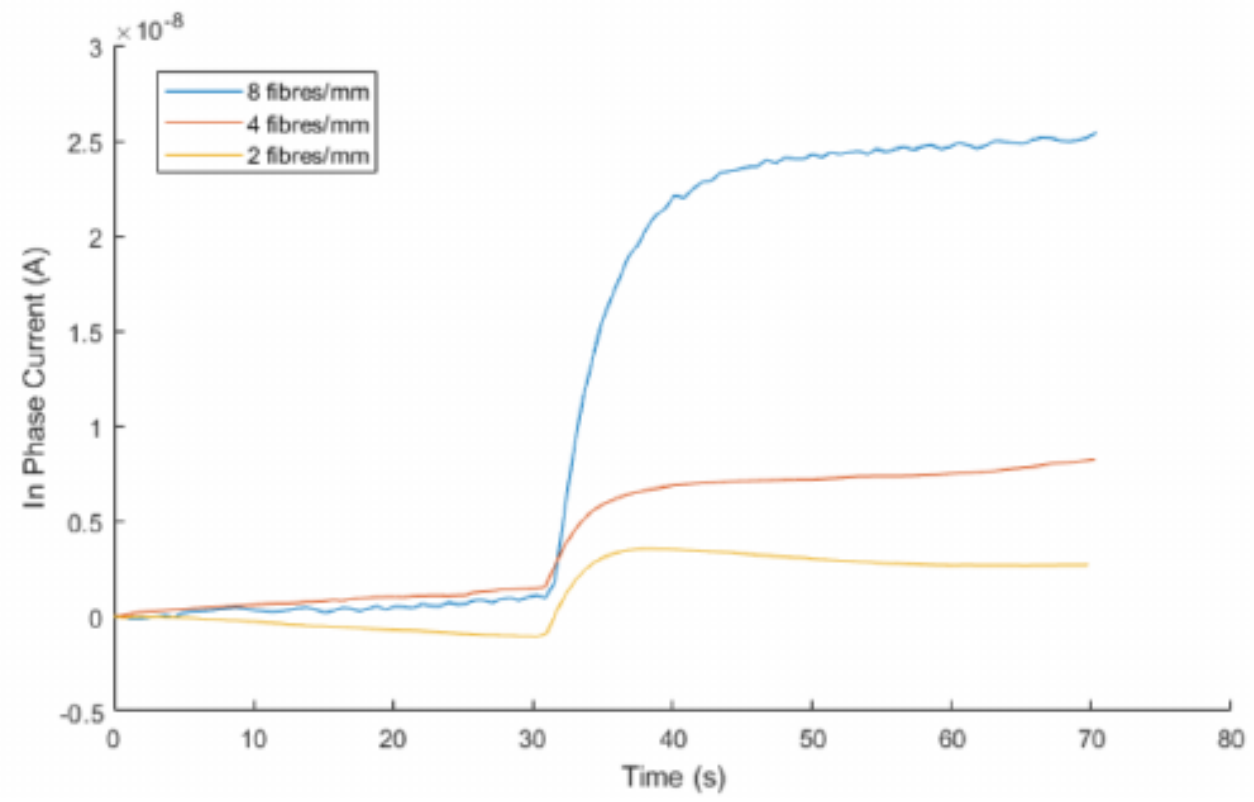

Figure 4.15: The conductance is observed to be constant before the application of a magnetic field at 30 seconds. After the field application, the conductance has a distinct increase as a result of fiber rotation. The long term heating effects and the glycerol-water component of the in-phase current are removed in this example. 


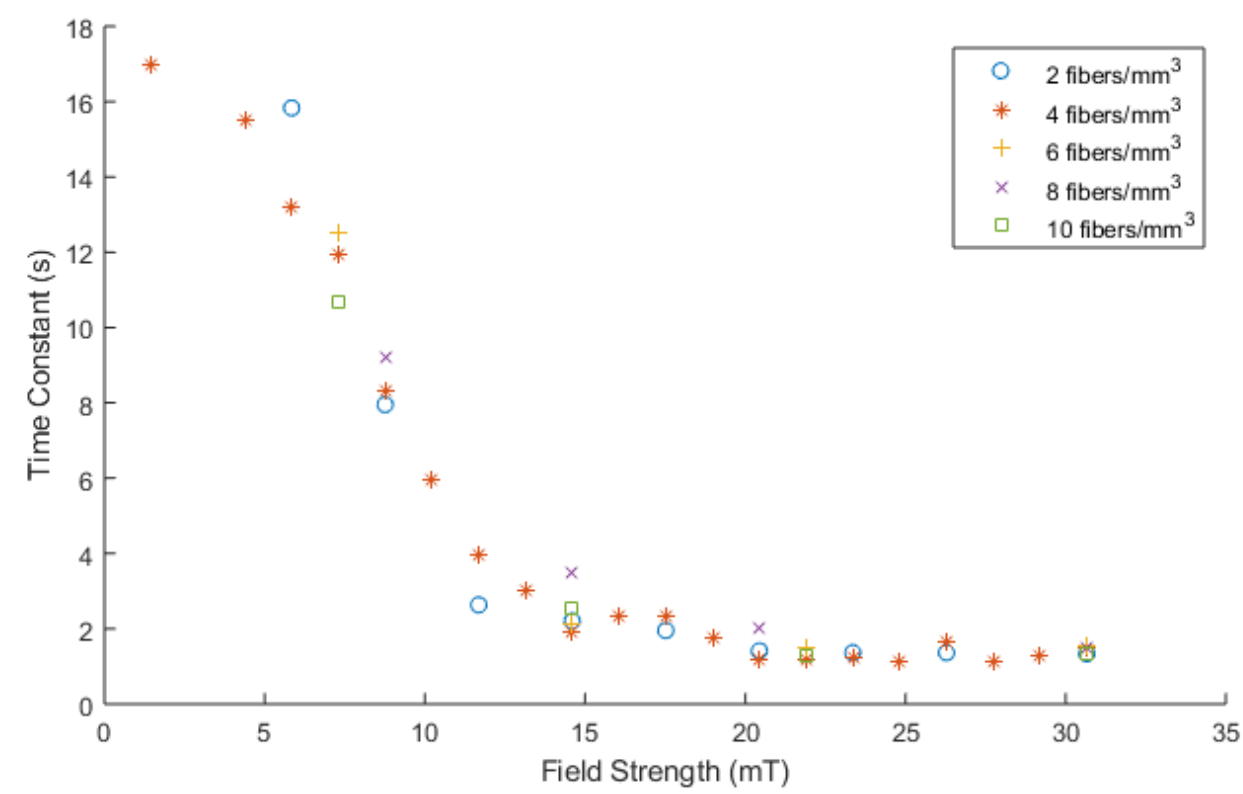

Figure 4.16: The time constant of conductance for fibers suspended in a $35 \pm$ $2 \mathrm{mPas}$ glycerol-water solution is observed to be independent of fiber concentration. 


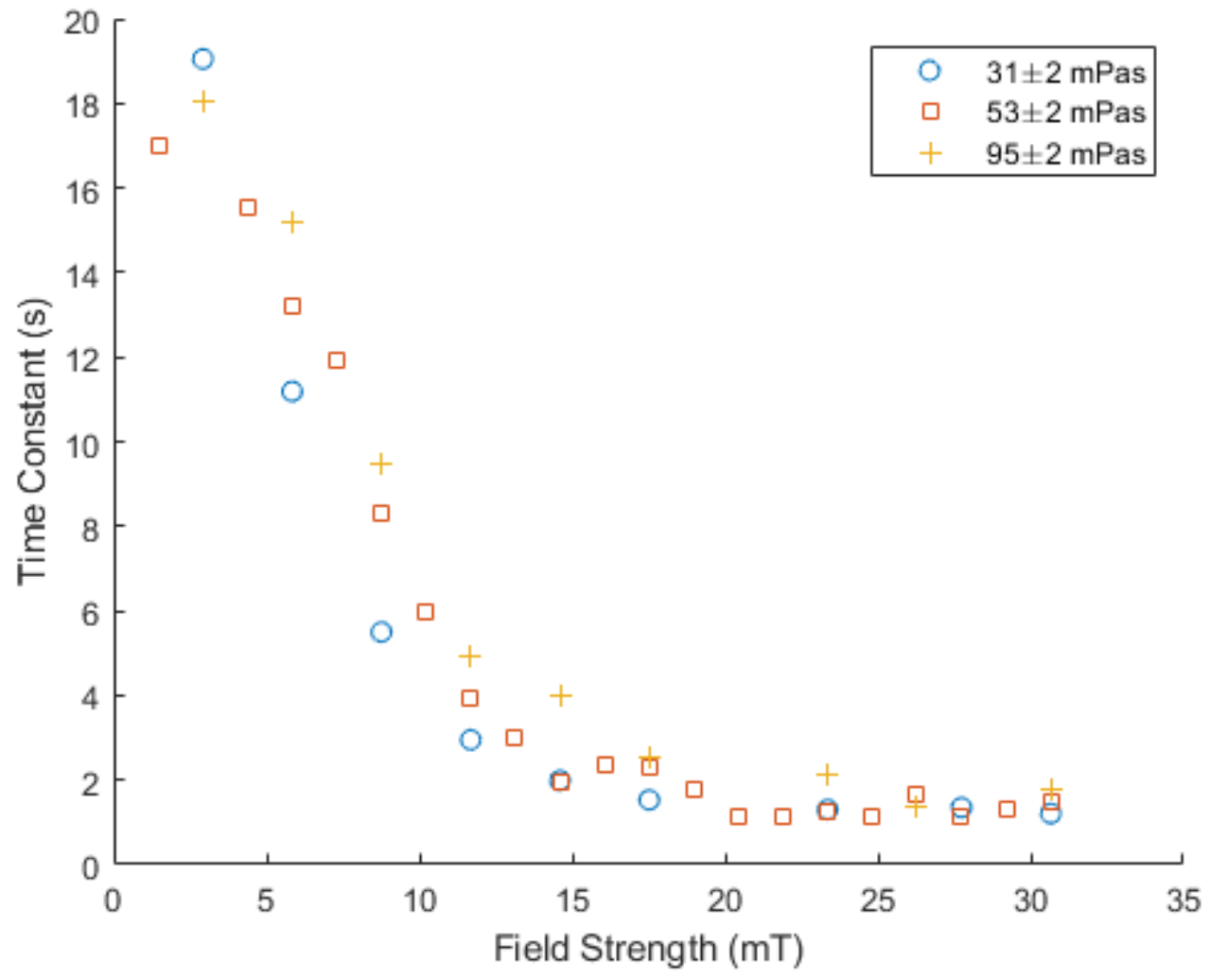

Figure 4.17: An increase in viscosity results in an increase in the conductance time constant when measured using the ring electrode configuration. 
This is illustrated in Figure 4.18, where the time constant of fiber rotation is compared to the conductance time constants for fibers in magnetic saturation. For a Newtonian fluid the viscosity time constant relationship should be linear, however the data presented is non-linear with viscosity. That said, Figure 4.5 illustrates the accuracy of the when adjusted for this non-Newtonian behaviour for a single randomly selected fiber.

The electrode configurations themselves were implemented to measure the time constant of the change in conductance, which may not be identical to the time constant for fiber rotation due to the geometry of the electrodes. This geometry effect is illustrated in Figure 4.18 where the two electrode configurations have different time constants for identical viscosities. However, a comparison can be mode between these different time constants once adjusted for the effects of the electrode geometry. The conductance time constants are observed to be scalable to align with the optical fiber rotation time constant, with scale factors of 0.5 for the quadrant electrode configuration, and 0.6 for the ring electrode configuration.

The non-linearity with viscosity is believed to be a product of the fluid motion induced through the rotating fibers. This introduces additional hydrodynamic torque on the fibers and hence increases the time constant of rotation. The lower viscosity solutions are observed to be more susceptible to this effect.

The analysis of electrode techniques shows the quadrant configuration is the most reliable method for measuring the fiber rotation time constants. This is primarily due to the superposition of magnetic fields present in the ring electrode configuration resulting in no distinct difference in the rotation time constant for different fluid viscosities. However, both methods are less reliable compared to optical analysis due to the required conversion between the change in conductance to obtain the fiber angle, and optical analysis should be implemented where possible. 


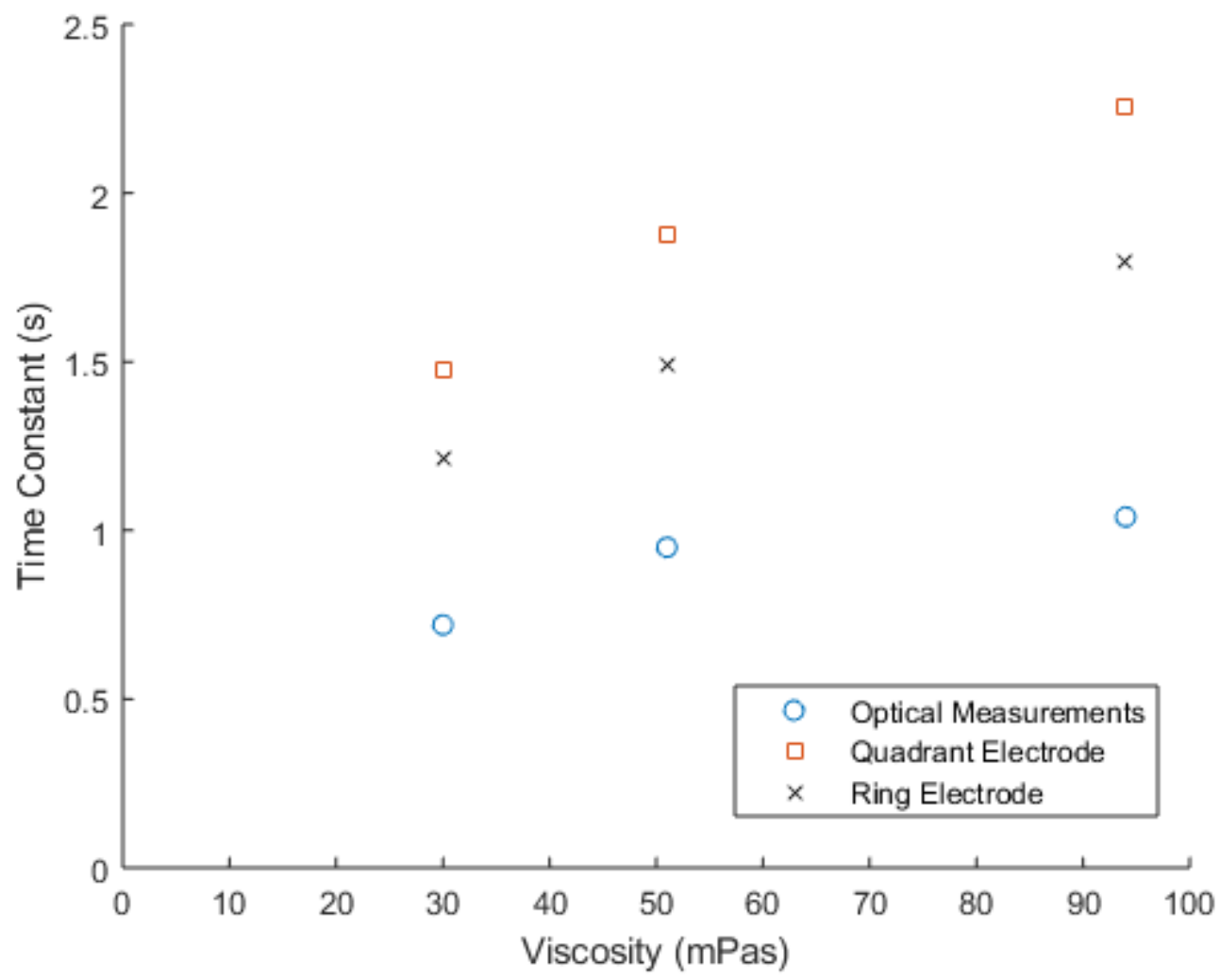

Figure 4.18: The time constants for conductance changes as measured by the quadrant and ring electrodes are observed to be scalar multiples of the optical time constants. 


\section{Chapter 5}

\section{Fiber Translation}

In order to produce porous piezoceramics fibers must settle to the bottom of a mold before the ceramic slip hardens and the fibers become trapped. Therefore the in-situ monitoring of fibers as they move through the mold and collect in the bottom of the mold is essential for the development of these piezoceramics. As mentioned in Section 2.4, experiments and modeling of fiber motion in stagnant and turbulent flow are not well described in literature, hence it is the aim of this chapter to present a mathematical model for the magnetically assisted settling of fibers. The results of optical monitoring of the fiber motion will then be presented. The monitoring of fibers using the electrode configuration presented. Finally, the fiber motion captured through these three methods are compared.

\subsection{Modeling of the Magnetically Assisted Sedi- mentation of Fibers}

In order to simulate the settling time of the fibers translation is considered independent of rotation, and they are considered to be vertically aligned. This allows for a simple force analysis to be completed on the fibers as they move through a magnetic field. The net force acting on a fiber is a combination of gravitational forces, drag, buoyancy, and magnetic attraction. The gravitational 
force is described as

$$
F_{\text {gravity }}=m a
$$

where $m$ is the mass of a fiber and $a$ is the acceleration due to gravity. The buoyancy force is described by

$$
F_{\text {buoyancy }}=\pi r^{2} l \rho
$$

where $r$ is the fiber radius, $l$ is the length of a fiber, and $\rho$ is the density of the fluid suspension. The drag force is calculated assuming the fibers are aligned with the magnetic field, hence is described by

$$
F_{\text {drag }}=\frac{2 \pi n l U}{(\ln (2 D)+C 1)}
$$

where $n$ is the fluid density, $U$ is the fiber velocity, $D$ is the fiber aspect ratio, and $\mathrm{C} 1$ describes the drag coefficient for a slender body [120]. The magnetic force acting on a fiber is described by

$$
F_{\text {magnetic }}=M \frac{d B}{d Z}
$$

where $M$ is the magnetic moment of a fiber and $\frac{d B}{d Z}$ is the magnetic field gradient. The equation for the net force is hence given by

$$
F_{\text {net }}=m a-\pi r^{2} l \rho-\frac{2 \pi n l U}{(\ln (2 D)+C 1)}+M \frac{d B}{d Z}
$$

Finally, substituting the fiber property values presented in Table 5.1 the net force becomes

$$
F_{\text {net }}=7.7060 \times 10^{-10}-1.3 \times 10^{-3} n U+2.36 \times 10^{-9} \frac{d B}{d Z}
$$

Using the magnetic field gradients in Figure 5.1, we can use the model to calculate the position, velocity, and acceleration of the fibers as they move through the mold. The field gradients selected for this analysis are $0 \mathrm{~T} / \mathrm{m}$ denoting the lack of a magnetic field, a linear magnetic field gradient of $100 \mathrm{~T} / \mathrm{m}$, the field gradient inside the enclosed magnetic cell, and the magnetic field gradient in the open magnetic cell. The linear and zero field gradients were selected as 


\begin{tabular}{|c|c|c|c|}
\hline Constant & Value & Constant & Value \\
\hline $\mathrm{m}$ & $8.38 \times 10^{-11} \mathrm{~kg}$ & 1 & $0.001 \mathrm{~m}$ \\
$\mathrm{a}$ & $9.81 \mathrm{~ms}^{-1}$ & $\mathrm{D}$ & 140.44 \\
$\mathrm{r}$ & $3.56 \times 10^{-6} \mathrm{~m}$ & $\mathrm{C} 1$ & -0.80685 \\
$\mathrm{M}$ & $2.46 \times 10^{-9}$ & $\rho$ & $1293 \mathrm{~kg} / \mathrm{m}^{3}$ \\
\hline
\end{tabular}

Table 5.1: Constants used in the fiber translation modeling.

a method of comparing the natural sedimentation of the fibers to the motion in the cell, and as a simple method to model if adding additional magnets to generate a linear magnetic field would have a distinct improvement in the sedimentation of the fibers. It is not practical to model the fiber motion in a negative field gradient as fibers in this space are expected to tend toward collecting in the top of the cell. An initial position of $10 \mathrm{~mm}$ from the top of the cell is selected to allow for the avoidance of this negative field gradient measured in the enclosed cell.

Fibers subjected to a larger magnetic fields gradient are observed to travel from their initial position to the bottom of the mold in $1 \mathrm{mPas}$ solutions, illustrated in Figure 5.2. Fibers not subject to a magnetic field exhibit a change in position linear with time arising from a constant velocity. The introduction of both linear and non-linear magnetic field gradients results in a non-linear distance profile. This is further illustrated in Figure 5.3 where the velocity of fibers subject to a magnetic field is non-linear, whereas the removal of the magnetic field creates constant velocity. The terminal velocity of these fibers not subjected to magnetic fields is reached immediately. There is no terminal velocity for fibers subjected to a magnetic field gradient which is not constant. This is because the magnetic force acting against drag increases as the fiber travels downward, increasing the fiber velocity resulting in an increase in drag. This feedback loop continues until the fiber can not travel further, and the velocity becomes zero. This effect is more drastic when the change in magnetic field gradient has a large increase with distance. This is seen through comparing the fibers velocity when subjected to the enclosed cell field gradient to the open cell 


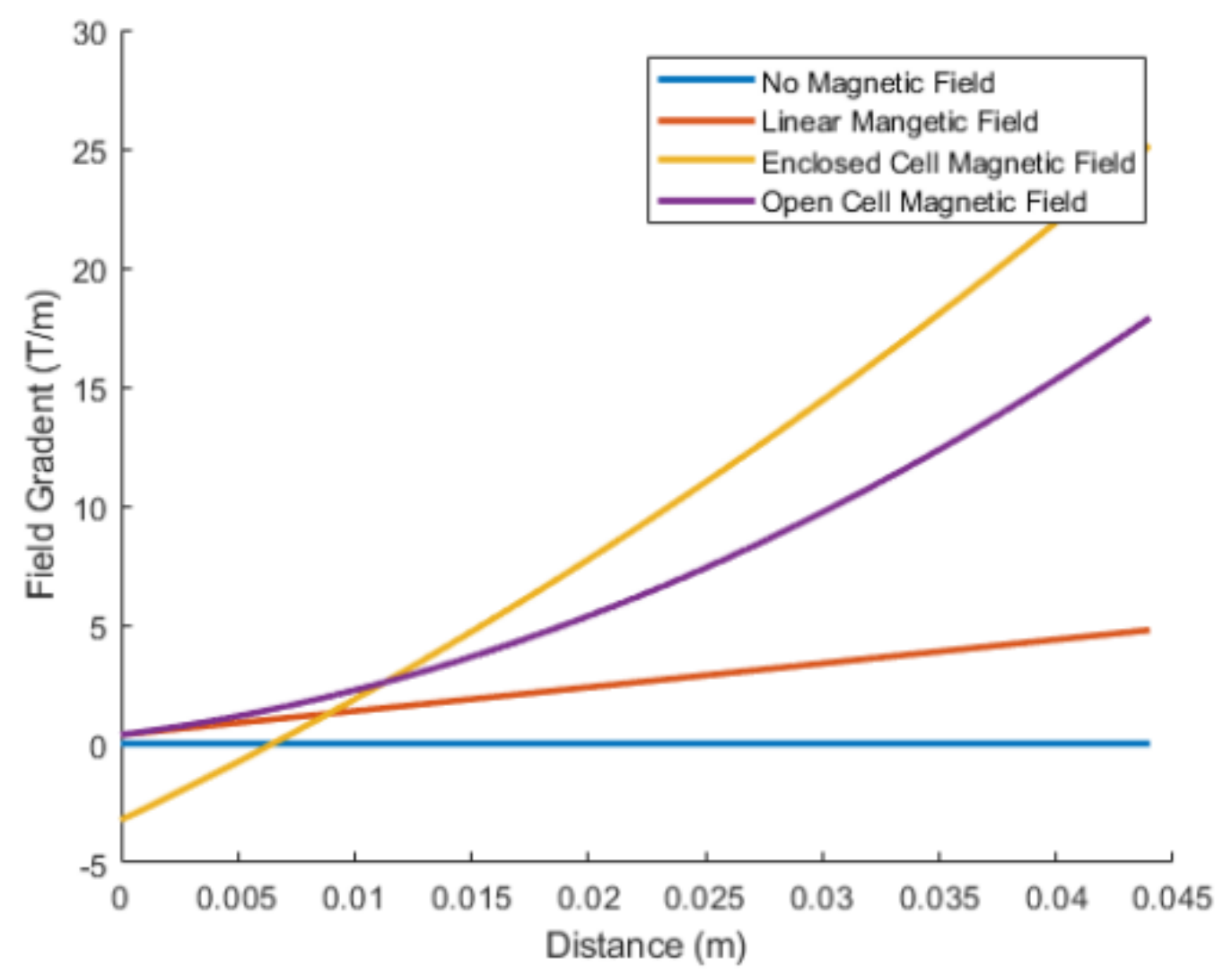

Figure 5.1: The magnetic field gradients used to model the fiber motion. The magnetic field gradient in the enclosed cell is negative in the top $6 \mathrm{~mm}$ of the cell. All other field gradients are entirely positive or zero. 
field gradient.

The acceleration of fibers is presented in Figure 5.4. An initial spike in acceleration at $\mathrm{t}=0$ is observed due to having zero initial drag force, and the fibers acceleration is solely a product of the magnetic force, gravitational forces, and buoyancy. For fibers nor subjected to a magnetic field gradient the acceleration is zero throughout the fibers motion. A linear magnetic field results in linear acceleration, and a non-linear field gradient results in non-linear acceleration. The maximum fiber acceleration obtained when the fibers reach the bottom of the mold is observed to be dependent on the magnitude of the magnetic field gradient.

The magnetic force and drag force illustrated in Figures 5.5 and 5.6 respectively both exhibit the same trends for the respective magnetic field gradients due to the dependance the drag has on the magnetic force. The magnetic force is greatest with high field gradients, resulting in high acceleration and velocity, and hence high drag. The inverse also holds, where low magnetic force corresponds to low drag. These forces are an order of magnitude greater than the combined gravitational and buoyancy forces when the fibers are $10 \mathrm{~mm}$ from the top of the cell at the beginning of the modeling results, which increases to two orders of magnitude when the fibers reach the bottom of the cell. This means both gravitational and buoyancy forces are negligible and can be effectively ignored, and due to the drag forces dependance on velocity, this leaves the fiber motion a product of the magnetic field gradient alone.

The effect of fluid viscosity on the fiber motion as fibers travel from the top to the bottom of the magnetic cells are presented in Figures 5.7 and 5.8. The fiber motion in the open magnetic cell begins at the top of the cell denoted when the distance is zero, and in the enclosed magnetic cell the fiber motion begins when the field gradient is positive at $10 \mathrm{~mm}$. In both cases, the time taken for fibers to travel from the top to the bottom of the cell is observed to be linearly proportional to the fluid viscosity.

Fibers in the enclosed magnetic cell are expected to collect in the top of the cell due to the negative field gradient, illustrated in Figure 5.9 by the reduction 


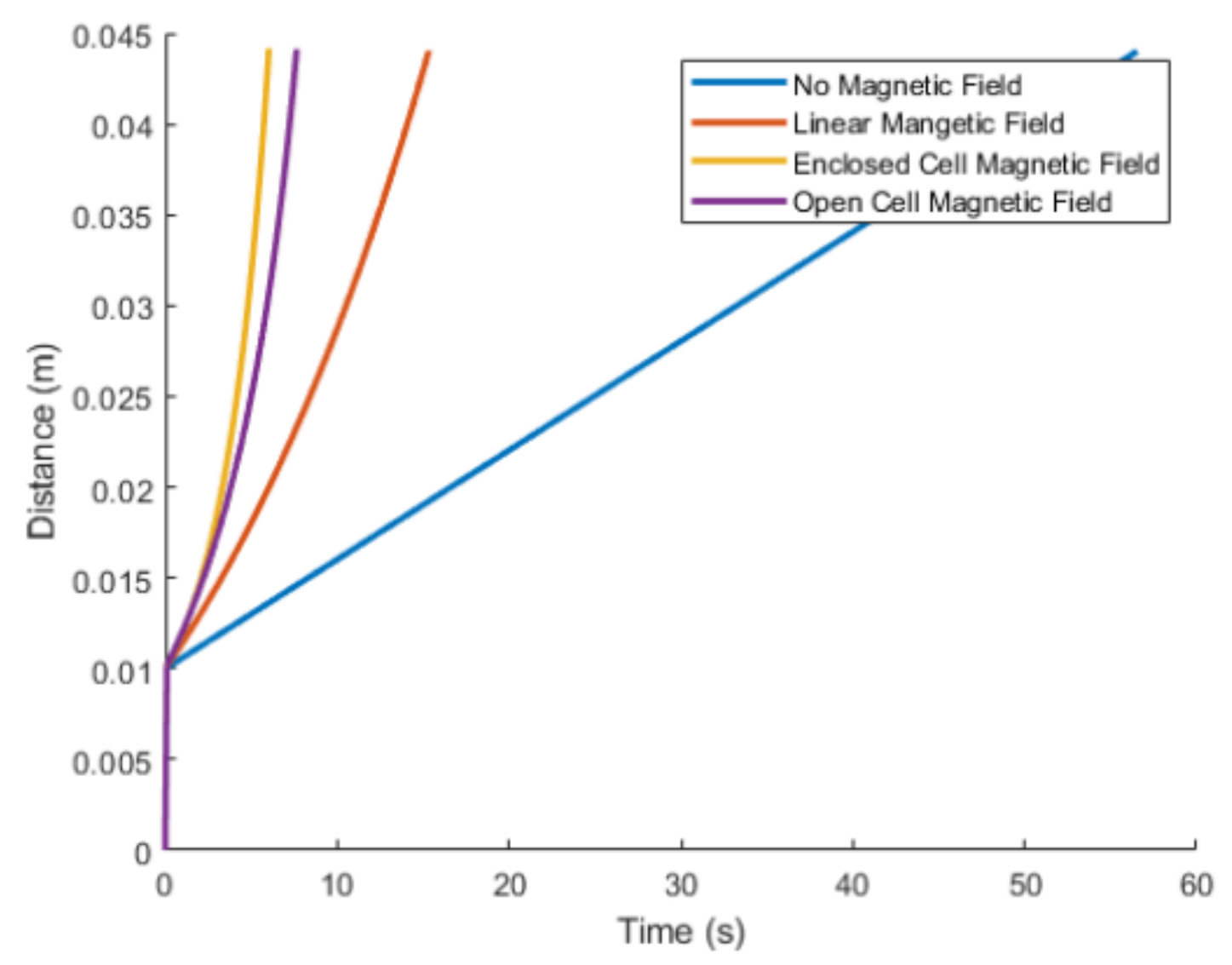

Figure 5.2: The model in Equation 5.6 shows fibers subject to high magnetic field gradients travel from their initial position at $10 \mathrm{~mm}$ to the bottom of the mold at $44 \mathrm{~mm}$ much more rapidly than low field gradients when suspended in a $10 \mathrm{mPas}$ solution. All field gradients are observed to improve upon the gravitational settling of the fibers. 


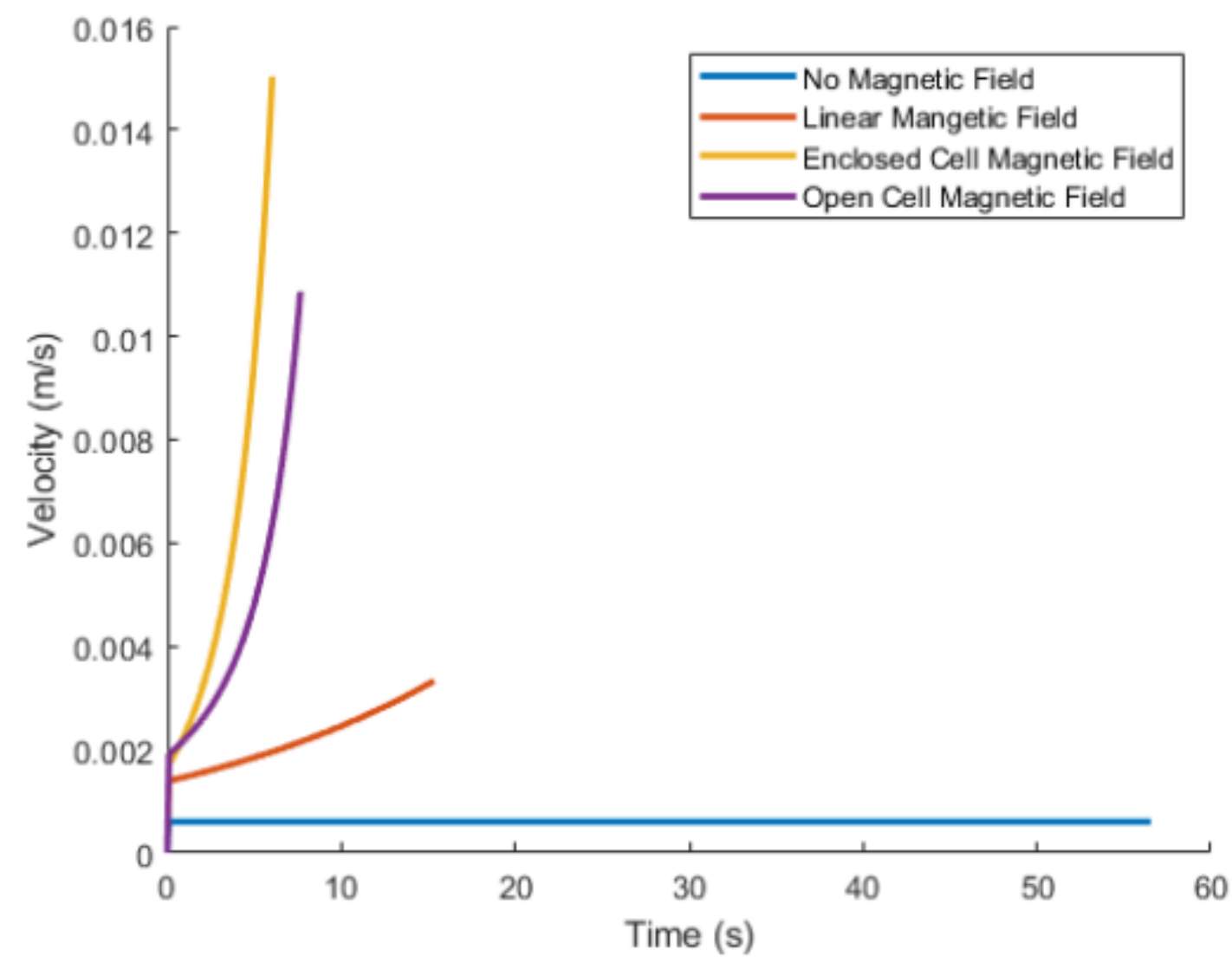

Figure 5.3: Modeled fibers not subjected to magnetic field gradients reach terminal velocity almost instantly resulting in a constant velocity. An increasing magnetic field gradient removed the ability for fiber velocity to saturate due to a force feedback loop. 


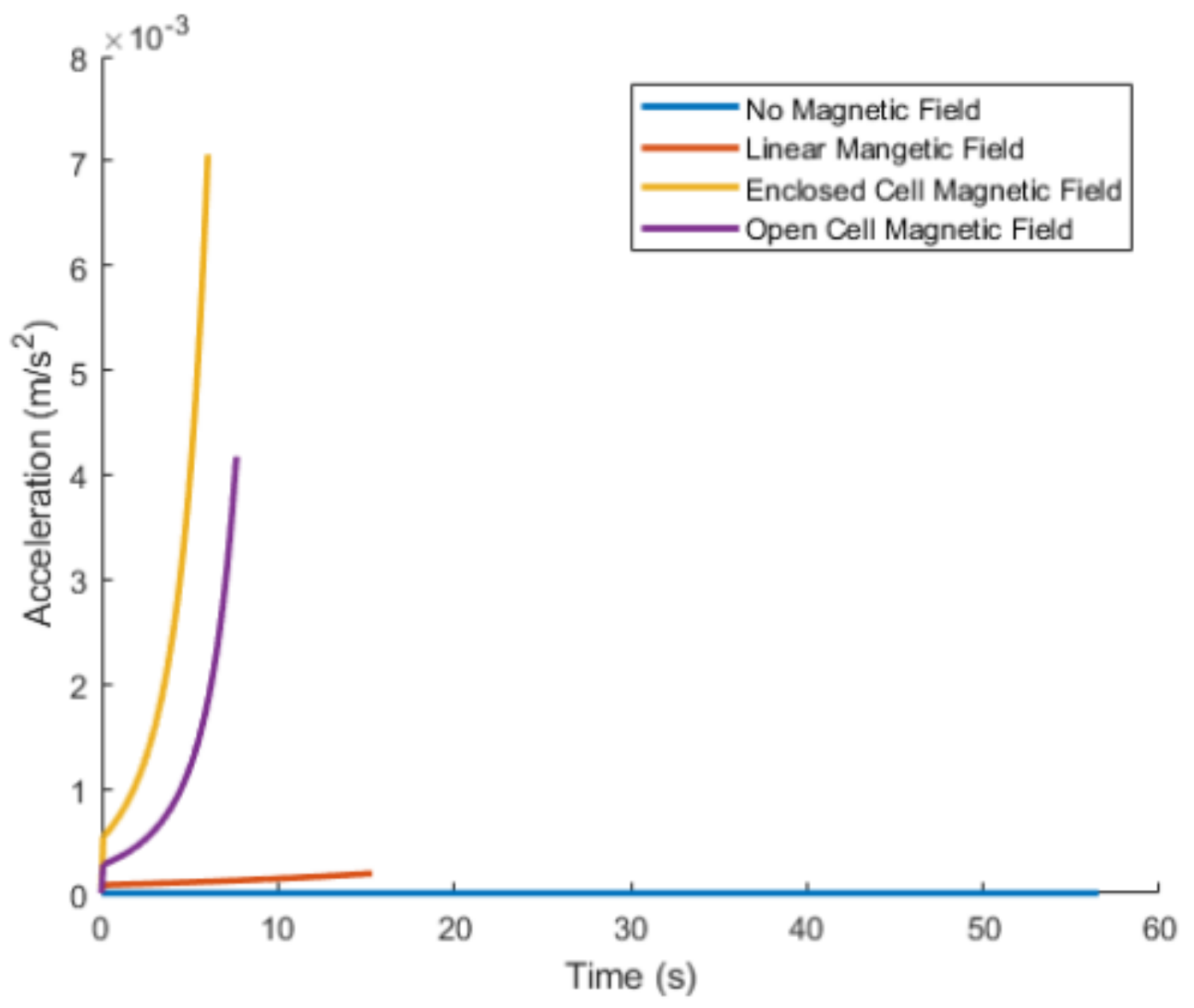

Figure 5.4: The modeled acceleration of fibers subjected to a non-linear magnetic field is non-linear and dependent on the magnitude of the field gradient. A linear magnetic field results in a linear acceleration, and zero acceleration is a product of no magnetic field gradient. 


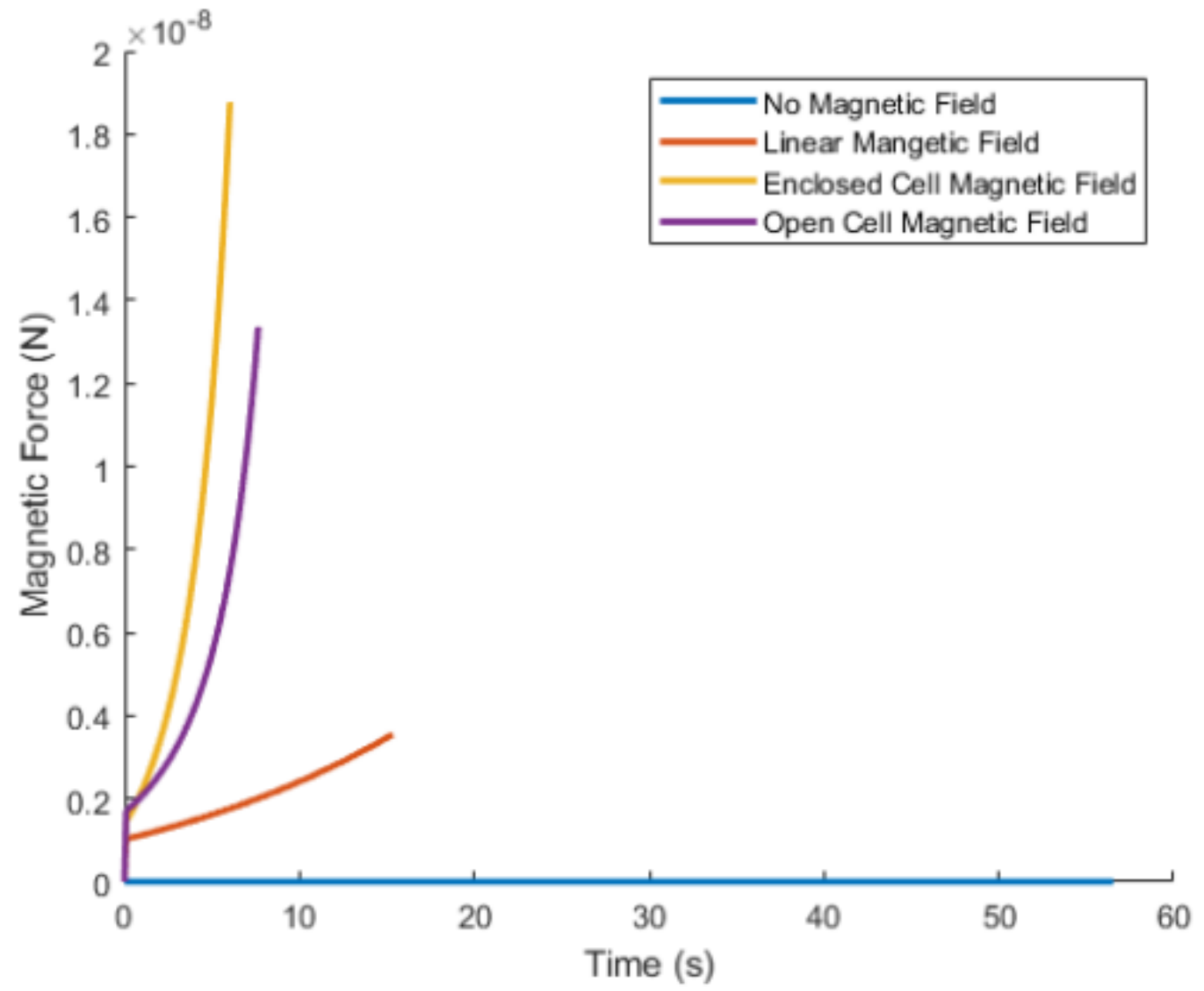

Figure 5.5: The magnetic force acting on a fiber as it travels through the cell is directly proportional to the field gradient. 


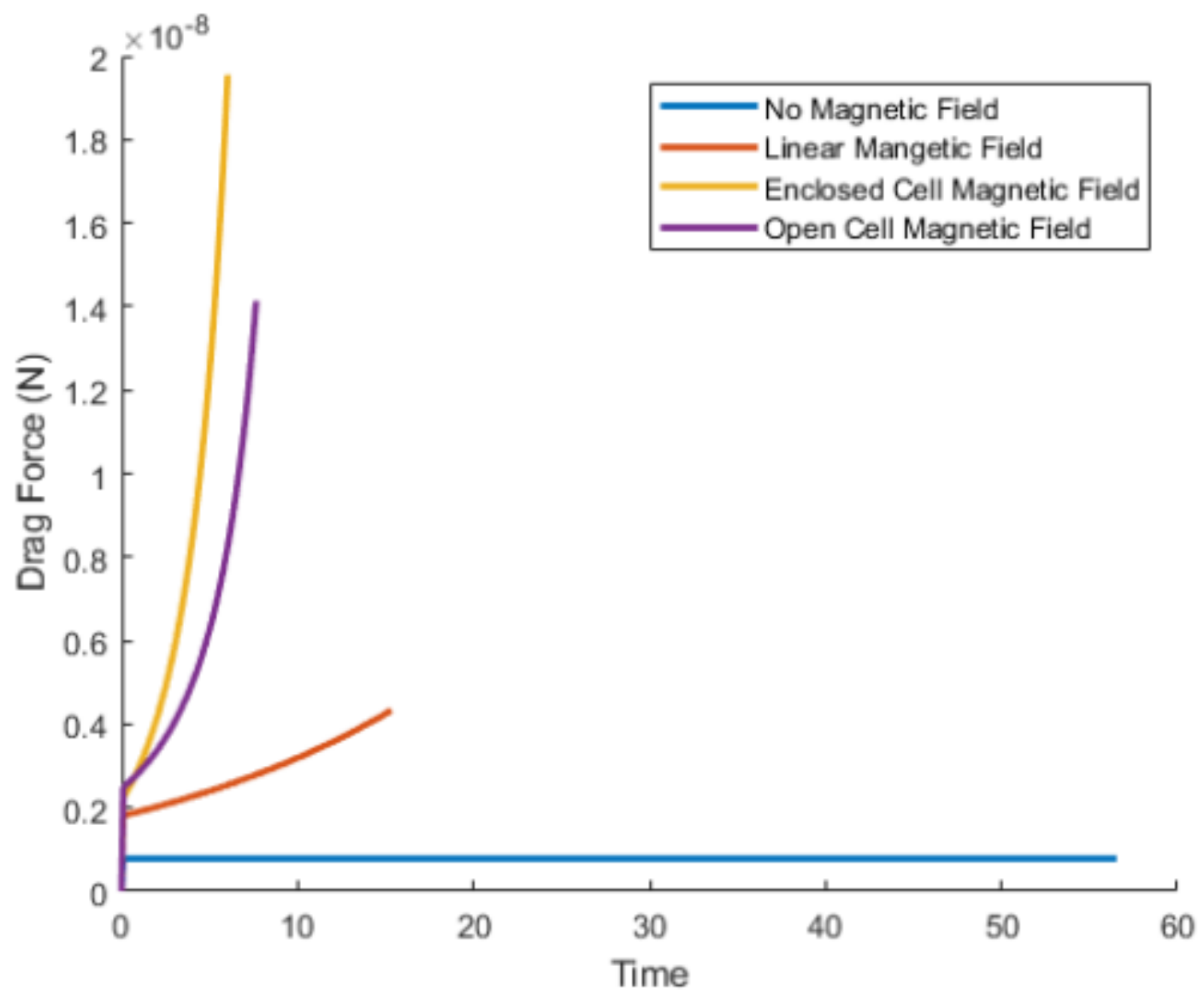

Figure 5.6: The drag force acting on a fiber is proportional to the fiber velocity, which is a product of the magnetic force. Thus high magnetic forces correspond to high drag forces. 


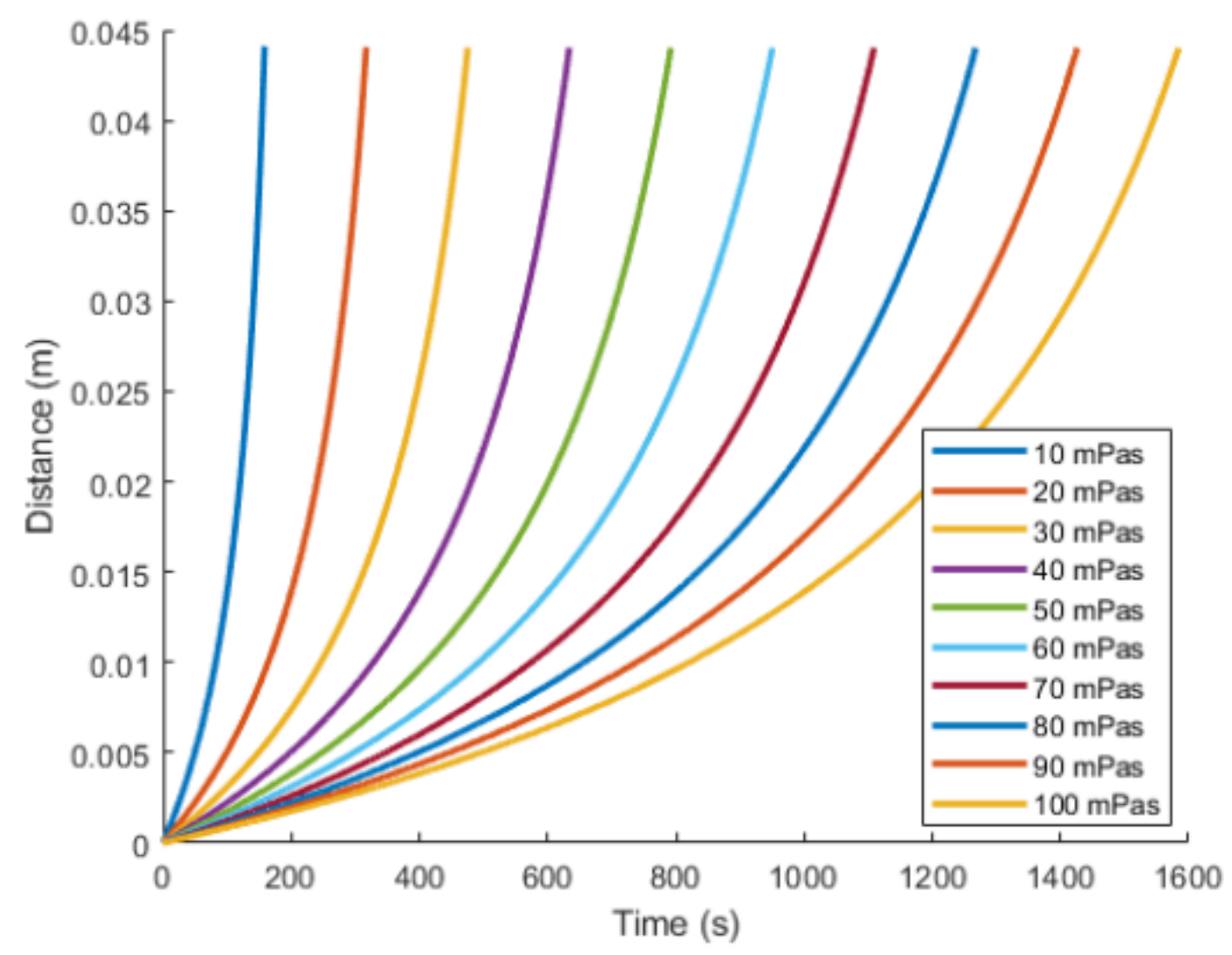

Figure 5.7: The modeling of fibers in the open magnetic cell predicts the time taken for fibers to travel from the top of the cell to the bottom to be linearly dependent on fluid viscosity. This is typical of particles moving in a Newtonian fluid. 


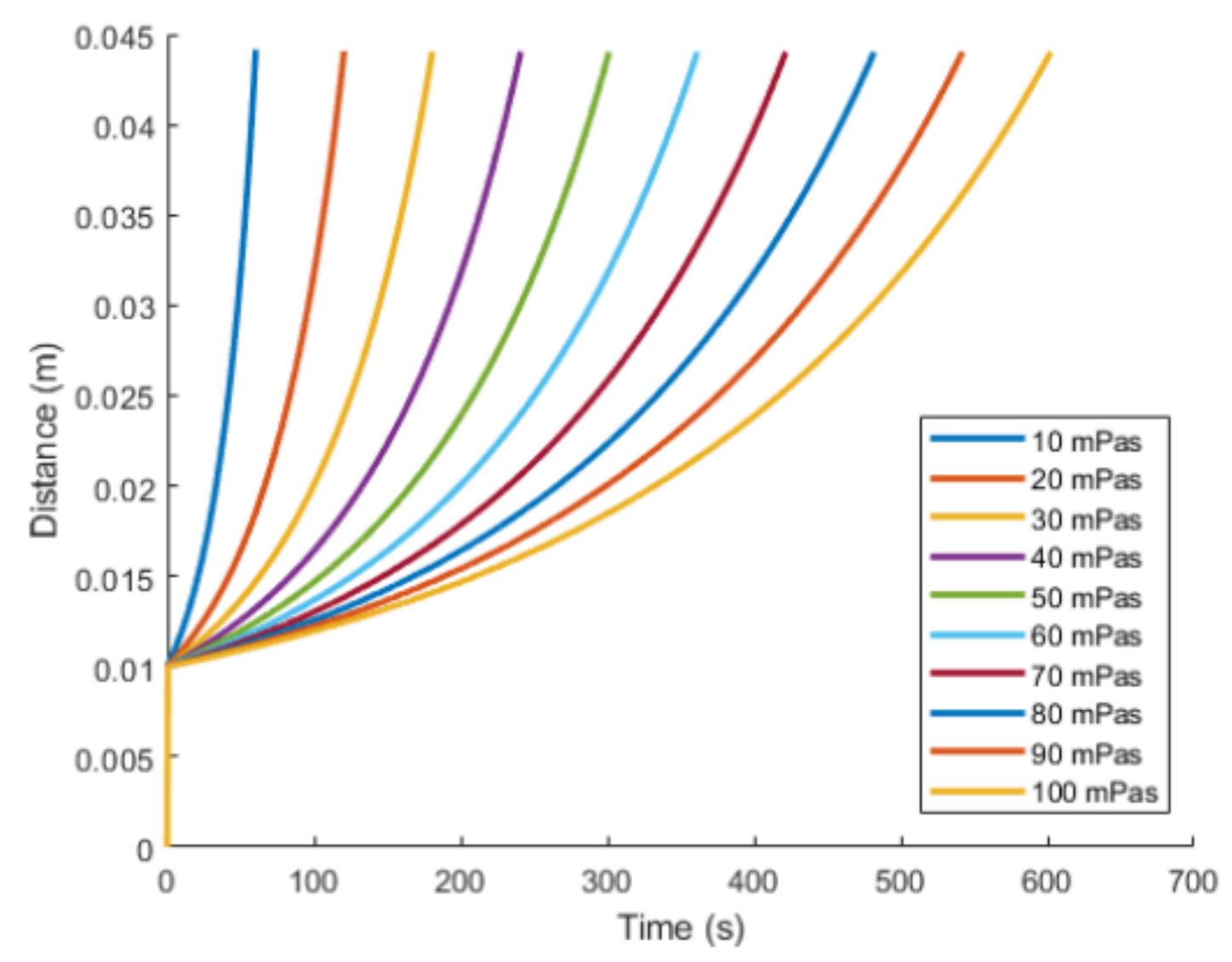

Figure 5.8: The modeled time taken for fibers to travel from $10 \mathrm{~mm}$ from the top of the enclosed magnetic cell to the bottom of the cell is linearly dependent on fluid viscosity. This omission of the top $10 \mathrm{~mm}$ of the cell is accounting for the region containing a negative field gradient present in the enclosed magnetic cell. 
in fiber position over time. The fiber motion is observed to have a strong nonlinear dependance on initial position in the enclosed cell. This is due to fibers closer to the bottom being subjected to a stronger magnetic field gradient and hence accelerate significantly faster than fibers at $6 \mathrm{~mm}$ from the top of the cell where the field gradient is close to zero. This gradient change creates a theoretical stagnation point where the forces acting on the cell entirely cancel and fibers lose all motion. The modeling suggests this point is between $0.004431 \mathrm{~m}$ and $0.004432 \mathrm{~m}$ from the top of the cell.

The model can be extended to a Monte-Carlo simulation through randomly generating a starting height for a number of fibers equal to the desired number density in the solution using a linear distribution. The fiber concentration at a desired height can then be calculated based on the fiber position over time. This models the expected response of the ladder electrode array for linear motion through defining the desired height as the height between the top of the top electrode and the bottom of the bottom electrode. The fiber concentration over this range can then be averaged, and compared to the electrical conductance measurements. This direct comparison method is suboptimal, and one can only compare the trends in the model and conductance measurements due to the complex geometry of the electrode configurations. In order to improve the analysis a complete mapping between fiber concentration and measured conductance which also takes into account temperature effects is required.

For mass fiber analysis the initial fiber velocity is considered to be zero. In reality this is not the case due to the combination of vertical laminar flow and stagnation point flow during fluid injection. However, due to the turbulent flow when the fluid contacts the bottom of mold, the individual fiber velocity and acceleration is random in both the vertical and horizontal plane.

Modeling the average fiber concentration between the top of the upper electrode and bottom of the lower electrode for each electrode pair of the ladder electrode configuration is presented in Figure 5.10. The model predicts fibers will exhibit a wave front type clearing, where the fiber concentration exhibits two distinct patterns. Firstly, the fiber concentration reduces exponentially as 


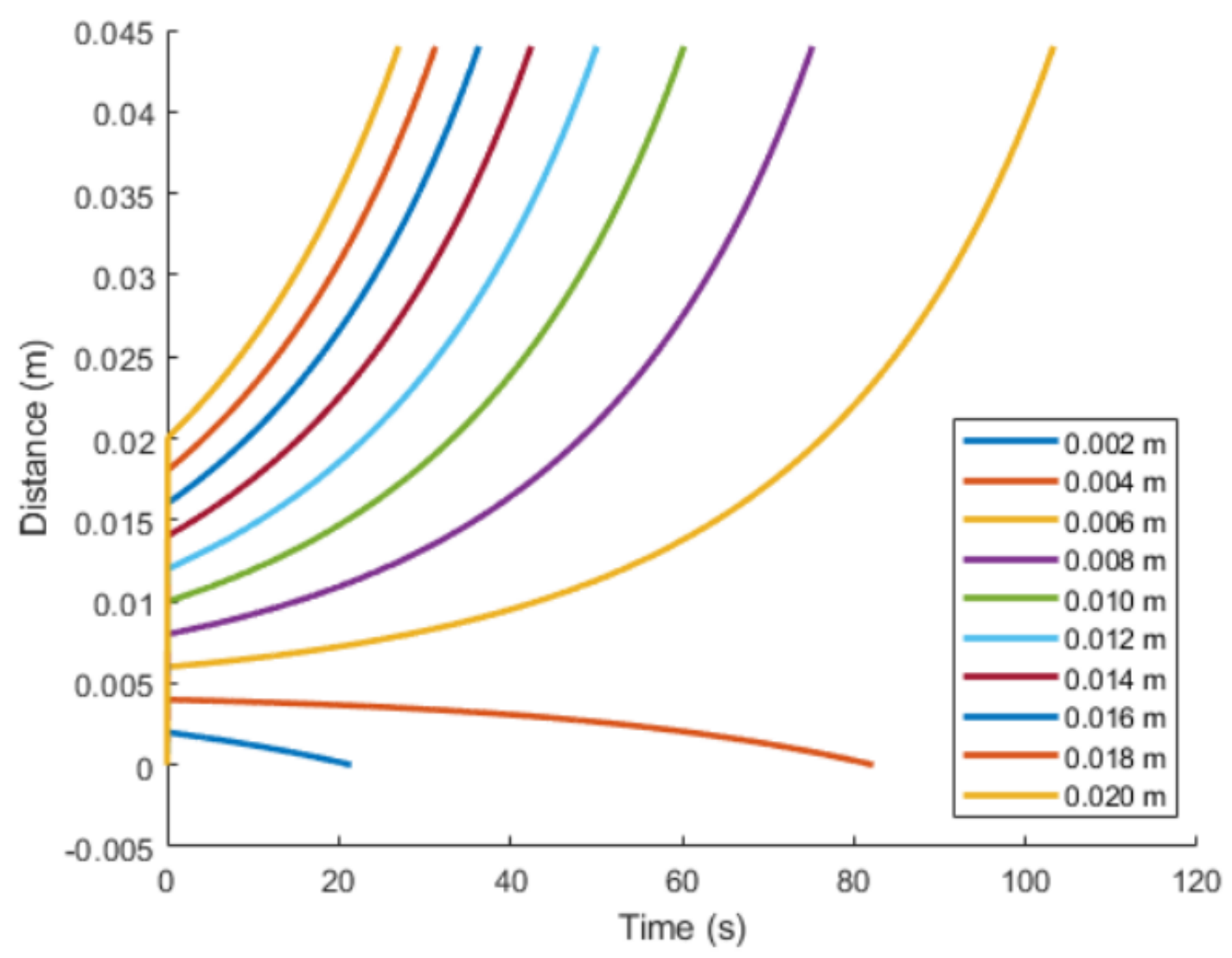

Figure 5.9: The fiber motion in the enclosed cell is observed to be non-linearly dependent on the initial fiber position. This is a product of two factors. Firstly, the region containing a negative field gradient which attracts fibers in the top $5 \mathrm{~mm}$ of the cell upwards to the top of the cell. Secondly, non-linearity in the field gradient in the bottom of the cell results in continuous acceleration towards to the bottom of the cell, with no terminal velocity reached. 


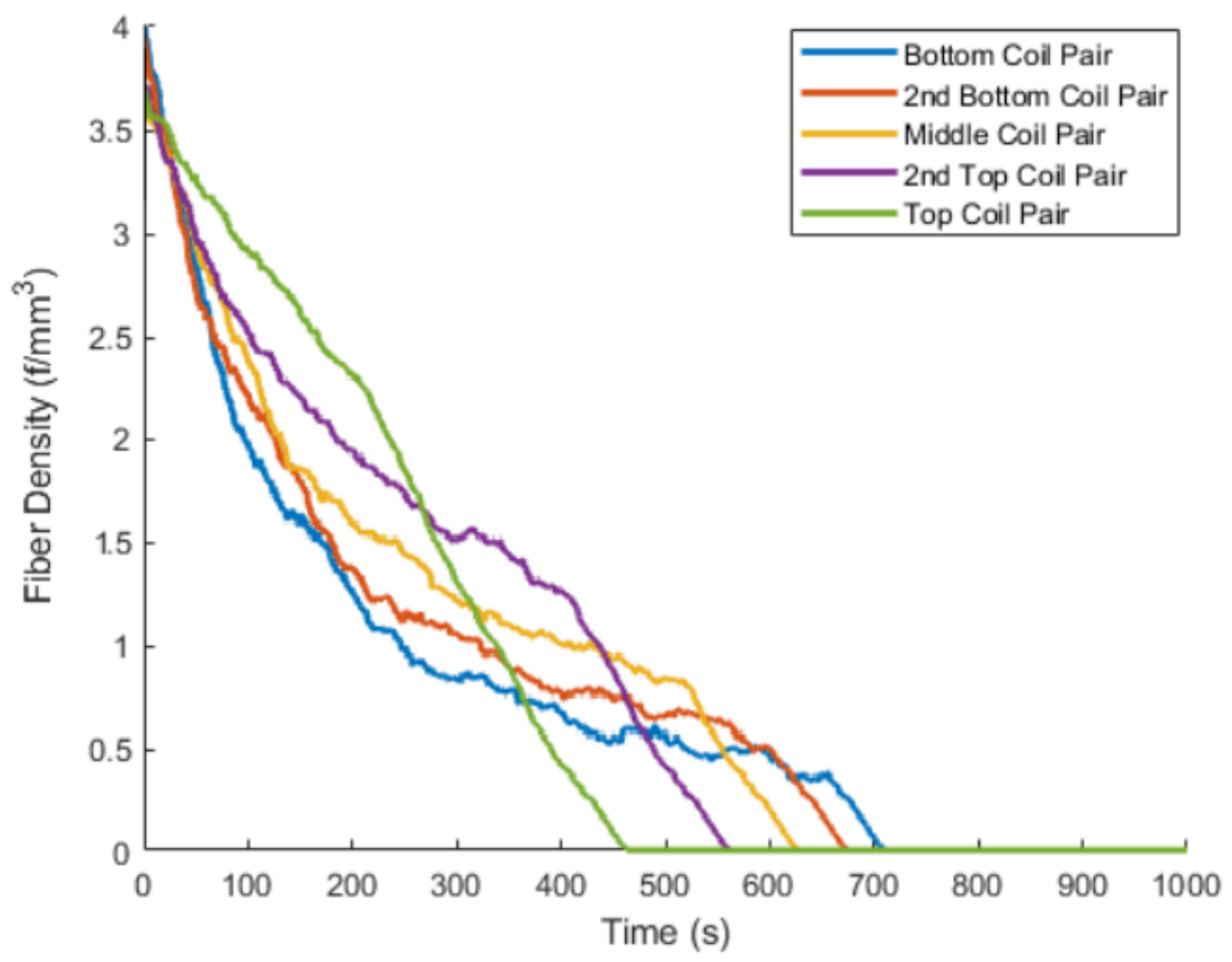

Figure 5.10: The modeled settling of fibers in the ladder electrode array can be simulated, giving the expected response from the measurement system. The initial fiber density was modeled to be 4 fibers per cubic millimeter, with the modeled suspension fluid having a viscosity of $50 \mathrm{mPas}$. The top $5 \mathrm{~mm}$ of the magnetic cell was omitted. 
they accelerate toward to bottom of the mold. Secondly, as the final fibers pass through the sensing region the fiber concentration rapidly decreases until there are no fibers in the sensing region. This wave front behaviour is expected in systems of non-interacting fibers, but does not account for high concentrations of fibers interacting physically or magnetically. That said, the model predicts fibers pass through the topmost electrode pair first but with a lower average velocity, and last through the bottom electrode pair but with a grater average velocity. This is consistent with an increasing magnetic field gradient at the bottom of the mold.

\subsection{Visual Observations of Fiber Translation}

In order to verify the numerical modeling of magnetically assisted fiber translation, visual observations were conducted. A suspension containing 4 fibers $/ \mathrm{mm}^{3}$ in a $50 \mathrm{mPas}$ and a $30 \mathrm{mPas}$ glycerol-water solution was created and injected into two small vials. Each vial was placed in the magnetic cell and the fiber position was optically monitored over a period of time, illustrated in Figure 5.11. The initial fiber orientation is approximately random, however there is some flow alignment observed due to the fluid motion during injection.

The degree of fiber clearing is calculated through analysing the quantity of visible fibers. This is achieved through comparing the quantity of pixels in a frame which are darker than a given threshold to those lighter than the threshold. These dark pixels denote fibers, whereas light pixels denote the lack of fibers. Thresholding in this manner in uncommon due to the superposition of objects in the frame making it difficult to identify individual objects, however it is a reproducible and reliable technique when fiber concentration is kept sufficiently low that there is approximately 50\% dark and 50\% light pixels, and the bottom section of the image where the fibers collect is ignored.

Rapid fiber settling is observed during the first 4 minutes of magnetic field exposure, with approximately $75 \%$ of the fibers cleared to the bottom of the vial. Fiber settling was determined to have completed after 12 minutes, where the 

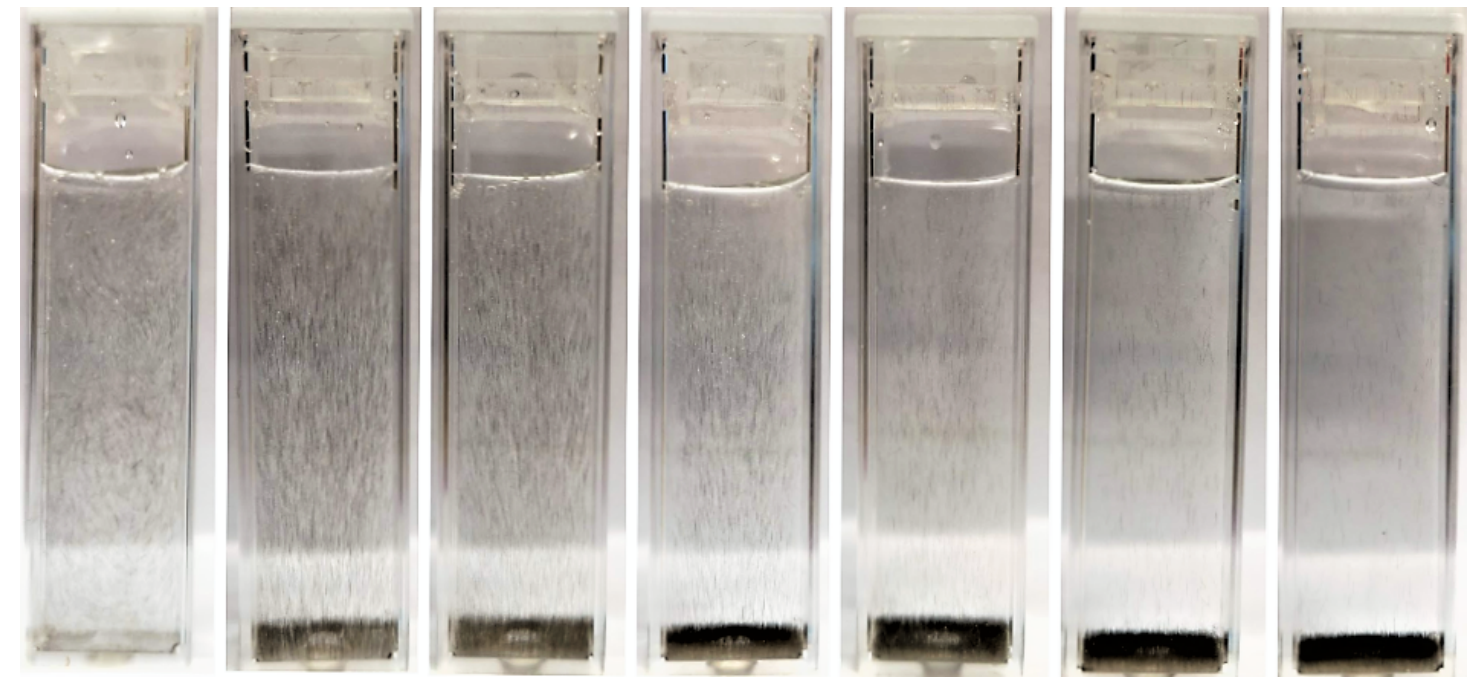

Figure 5.11: Contrast adjusted visual observations of settling times in $45 \mathrm{mPas}$ glycerol water solutions after $0,1,2,3,4,6$, and 12 minutes in magnetic field (from left).

remaining fibers show no movement beyond this time and have likely became stuck to the inside walls of the vial. It should be noted the vial illustrated in Figure 5.11 is not completely full of the fiber suspension. This empty section of the vial contains both the fiber stagnation point and the negative field gradient.

An array of aligned fibers is observed to form in the bottom of the mold, illustrated in Figure 5.12a. The initial fiber concentration to create this degree of alignment is observed to be the upper limit to create a single layer of fibers at 12 fibers $/ \mathrm{mm}^{3}$. Increasing the fiber concentration beyond this limit results in multiple layers of aligned fibers at the bottom of the mold illustrated in Figure $5.12 b$, where a fiber concentration of 16 fibers $/ \mathrm{mm} 3$ results in three layers of magnetically aligned fibers. These fibers non-uniformly across the bottom of the mold due to injecting the fibers into the mold. The narrow stream of fluid creates a non-uniform fiber distribution in the mold, where directly below the injection point a higher fiber concentration region is formed, and at the mold walls is a lower fiber concentration.

The negative field gradient is theorised to result in the collection of fibers 


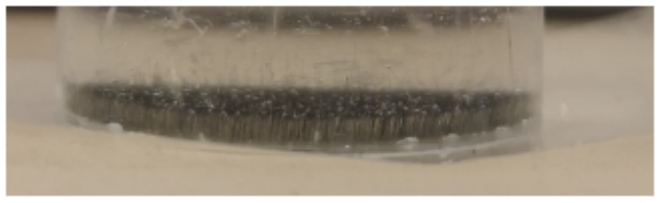

(a)

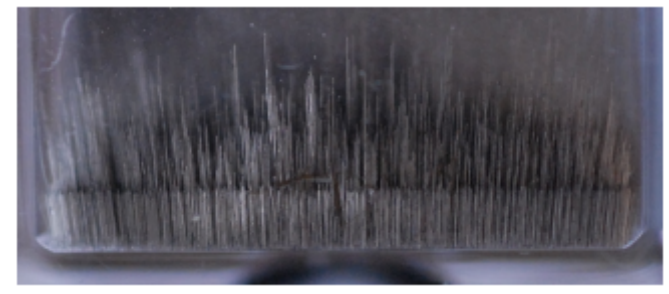

(b)

Figure 5.12: The limit for creating a single layer of aligned fibers was found to be 12 fibers $/ \mathrm{mm}^{3}$ (a). Increasing the fiber concentration beyond this limit results in multiple layers of aligned fibers (b).

at the top of the cell. This is observed in Figure 5.13 where fibers are stuck in the fluid meniscus and against the pole piece of the magnetic cell. Additionally, fibers appear to be stuck in the stagnation point where the magnetic field gradient changed from positive to negative. In order to prevent these effects during piezoceramic wafer fabrication leaving the top $5 \mathrm{~mm}$ of the mold devoid of fluid is one method. Another method is the addition of a second magnet to the cell to change the magnetic field gradient in the cell.

\subsection{Electrical Measurement of Fiber Translation}

In order to monitor the fiber density over time, a ladder electrode array was constructed which consists of a series of ring electrodes stacked on top of each other. However the in-phase current component of the ring configuration was observed to be independent of fiber density. As the addition of fibers changes the glycerol-water solution from being purely resistive to inductive and capacitive, the out-of-phase component of the current was used to monitor the fiber motion.

The results of implementing this inductive and capacitive sensing is illustrated in Figure 5.14, where the change in out-of-phase conductance is shown to reduce as a function of time. There are a number of key elements in the result which need to be discussed. Firstly, the spike in conductance at 80 seconds 


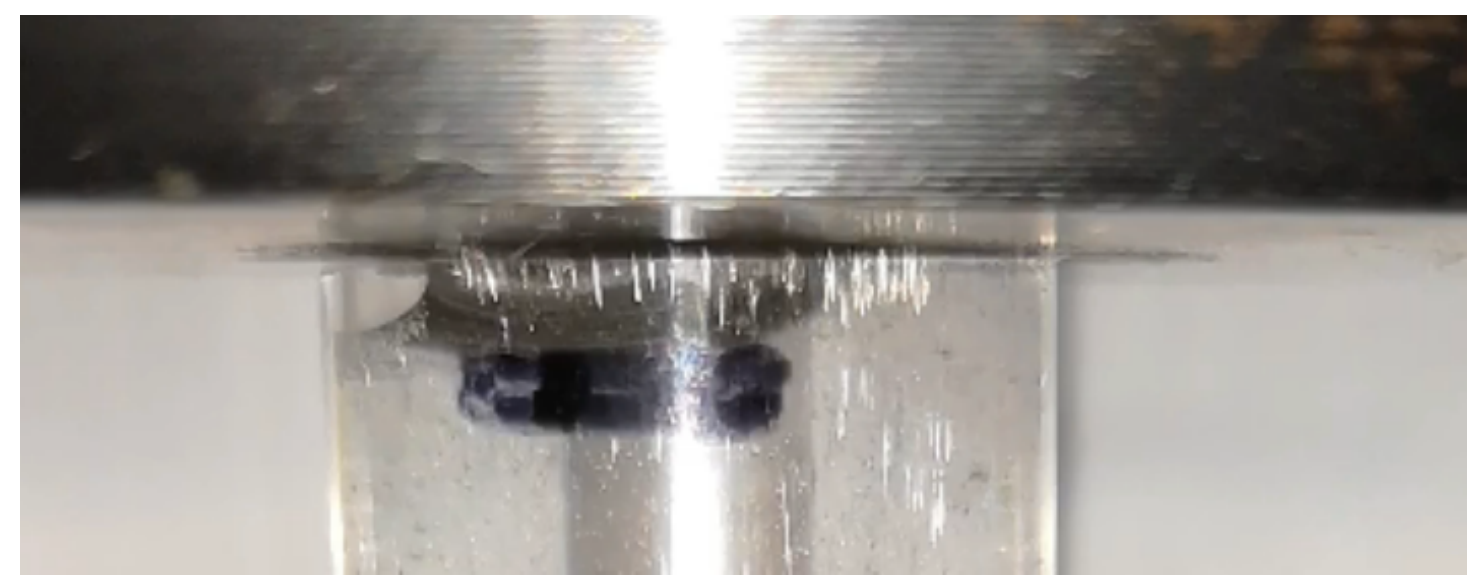

Figure 5.13: The negative field gradient in the top of the enclosed magnetic cell results in fibers collecting in the meniscus of the fluid, which is the opposite of the desired outcome.

denotes the time when the fibers suspended in the solution was injected into the mold and electrode array. The conductance immediately after the fluid is injected is observed to be different for each electrode pair. This is a product of the time taken for the multiplexor to iterate thorough the electrode array and record the conductance. Secondly, the conductance of the top electrode varies significantly with time. This is due to the fiber collection in the top of the cell, illustrated in Figure 5.13. The fibers become randomly released from the top of the cell due to external stimulus, resulting in the periodic influx of fibers through the top and 2nd top electrode pairs. Finally, the conductance measured by the bottom electrode is observed to reach a consistent level greater than that of the other electrodes. This is a result of the high density of fibers collecting in the bottom of the cell beside this electrode however outside of the direct sensing region.

The end of fiber motion is defined by the point at which the conductance measured across the bottom electrode pair no longer changes. In the example data illustrated in Figure 5.14 this point is at 250 seconds. However, due to the temperature sensitivity of these experiments and fibers stuck at the stagnation point which are freed by external stimulus, it is difficult to determine the exact 


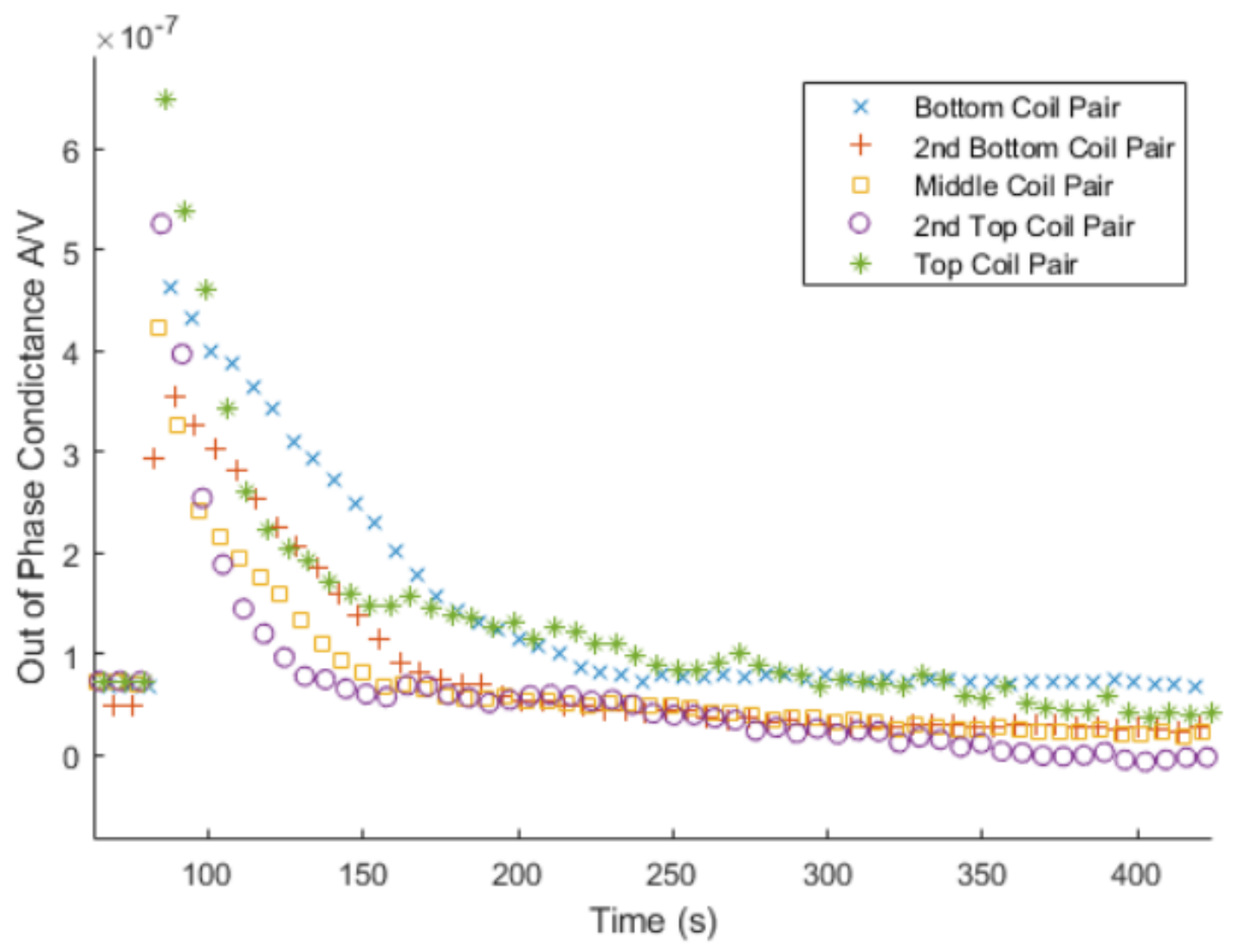

Figure 5.14: The change in the out-of-phase component of conductance over time suggests fiber motion in the cell. Fibers in the top of the mold are observed to clear quickly whereas it takes significantly longer for fibers in the bottom to clear. 


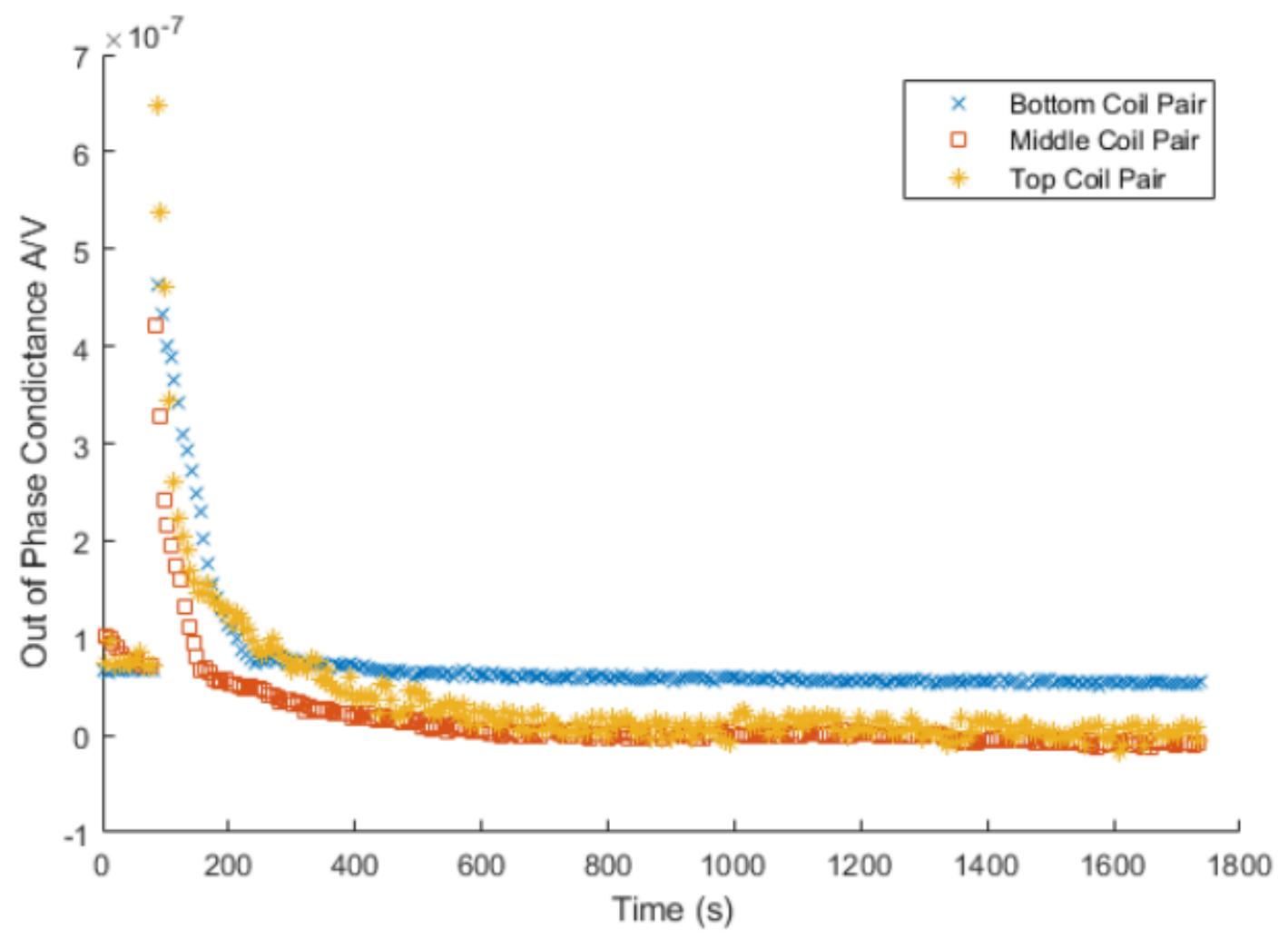

Figure 5.15: The fiber motion inferred by the bottom electrode pairs constant conductance ends at 400 seconds, whereas both the top and middle electrodes conductance becomes constant after approximately 750 seconds. Noise in the system in combination with imperfections in the electrode coils results in minor deviation in the steady state of all electrode coil pairs.

point at which fiber motion ends. Figure 5.15 illustrates the conductance measured by the top, middle, and bottom electrodes. The bottom electrode pair in this example reaches a stable level around 400 seconds, however the middle and top electrode pairs reach a stable conductance at approximately 750 seconds. As we are interesting in the fiber motion throughout the entire mold the point at which all electrode pairs reach a stable conductance will be used to define the clearing time. 


\subsection{Discussion}

A comparison between the methods for monitoring the translational fiber motion shows the time for complete clearing is varies significantly depending on the method used to measure or infer it. These differences are presented in Table 5.2, where the modeling results and optical results are in good accordance, however the electrical monitoring results in clearing in approximately half the time when solely basing clearing off the bottom electrode. This difference may be attributed to the limited sensing region of the electrode and interference from the collection of fibers in the bottom of the mold. The ladder electrodes electric field used to measure the conductance of the fiber suspension has an area in the center of the mold which may be missed by this electrode design. This, in conjunction with the large number of fibers concentrated in this area from the injection process illustrated in Figure 5.12b may make the bottom electrode alone suboptimal for inferring the fiber motion throughout the entire mold. This difference in settling time may be due to the difficulty in defining the end point of fiber settling in the electrical experiments. Incorporating the other electrodes in the determination of the clearing time puts the electrical method in good accordance when using a high viscosity fluid. The results from all methods are approximately linear with viscosity which is expected of a Newtonian fluid. 


\begin{tabular}{|c|c|c|}
\hline Method & Viscosity (mPas) & Time (s) \\
\hline Modeling & 30 & $425 \pm 5$ \\
Optical & 30 & $300 \pm 20$ \\
Electrical (Bottom Electrode) & 30 & $170 \pm 10$ \\
Electrical (All Electrodes) & 30 & $315 \pm 10$ \\
Modeling & 50 & $710 \pm 5$ \\
Optical & 50 & $720 \pm 20$ \\
Electrical (Bottom Electrode) & 50 & $290 \pm 10$ \\
Electrical (All Electrodes) & 50 & $750 \pm 10$ \\
\hline
\end{tabular}

Table 5.2: Time taken for fibers to completely settle to the bottom of the mold in the magnetic cell. 


\section{Chapter 6}

\section{Summery and Conclusion}

This thesis aims to analyse existing techniques for measuring fiber dynamics and models for fiber rotation and translation in magnetic fields. Using this analysis, a non-optical method for measuring fiber rotation and translation was devised. The results of this non-optical method was compared to optical and modeling results in order to understand fiber dynamics when subjected to magnetic fields, with the intended application being the fabrication of porous piezoelectric ceramics.

The model developed by Shine et al. [68] balancing hydrodynamic torque and magnetic torque is in good accordance with the visual observations made using transmission microscopy when small adjustments are made to the modeled viscosity. The electrical techniques developed for fiber rotation uses a change in the in-phase component of conductance measured by an electrode, which corresponds to a change in fiber orientation. The geometry of the electrodes impacts the applied field, where the creation of a magnetic field due to the displacement of charged particles superimposes on the solenoid generated magnetic field. This superposition of fields decreases the conductance time constant, implying an increase in the time taken for fiber to align with the fields. However the model predicts an increase in fluid viscosity to result in an increase in the change in conductance time constant, which is somewhat lost when using the ring electrode configuration. For this reason, a quadrant electrode config- 
uration is recommended. A direct comparison between the change in conductance time constants obtained from the electrode configurations and the fiber rotation time constants obtained by optical methods is ill advised. However a scaling factor can be applied to each conductance time constant to compensate for the geometry and conductance to rotation conversions. A scale factor of 0.5 is appropriate for the quadrant electrode, and 0.6 for the ring electrode. The fluid solution used to suspend the fibers is Newtonian in nature, however all measurement systems found the time constants are non-linear with fluid viscosity. This is believed to be due to the fibers rotation creating small turbulence in the fluid, increasing the effective viscosity.

Models for magnetically assisted fiber translation are sparse in literature, so a model balancing gravitational, drag, buoyancy, and magnetic forces on a fiber was developed. The model is a good predictor for fibers suspended in viscosity solutions greater than $30 \mathrm{mPas}$ when compared to optically recorded fiber motion. An electrode configuration was developed to measure the fiber motion non-optically. The electrode configuration consists of a series of ring electrodes stacked vertically, which adds spatial diversity to a simple electrode system. Using the full range of electrodes, we can monitor the sedimentation of fibers in the magnetic cell, however noise in the system is noticeable when the fibers have cleared from the electrodes sensing region. A comparison between the optical and electrical measurements shows the electrode configuration is a reliable and accurate method for the in situ monitoring of the fiber sedimentation.

\subsection{Recommendations for the Fabrication of Porous Ceramics}

From the analysis of fiber dynamics throughout this project, a list of observations and recommendations regarding the fabrication of piezoelectric ceramics and other similar composite materials has been devised.

- The addition of fibers to a fluid results in an increase of apparent viscosity 
while fibers rotate. Flow alignment via the injection of the material into the mold reduces the angle fibers are required to rotate to create an array of aligned fibers.

- The maximum fiber concentration to create a single layer of aligned fibers in the bottom of the mold was found to be 12 fibers $/ \mathrm{mm}^{3}$. Increasing beyond this creates secondary and tertiary layers of fibers in the mold.

- The injection of fibers into the mold creates a non-uniform distribution in the bottom of the mold, with a high concentration of fibers directly below the injection point and a low concentration at the sides of the mold. Moving the mold or injection point during fiber motion will result in a uniform fiber density resulting in a uniform array of pores.

- Lower viscosity solutions result in faster fiber alignment and settling. $30 \mathrm{mPas}$ solutions require 300 seconds for an array of aligned fibers to form, compared to 720 seconds for $50 \mathrm{mPas}$ solutions. This puts an upper limit on the fluid viscosity depending on the ceramic casting process implemented.

\subsection{Future Work}

Techniques have been devised for the analysis of fiber motion in opaque solutions, however these techniques have generally only been applied to a transparent glycerol-water solution. The next step in this project is to characterise the ceramic slurry and apply the electrical technique used to monitor fiber motion. The ceramic slurries may be highly conductive due to the water content and PZT conductivity [121]. A single experiment attempting to measure the fiber rotation in a PZT ceramic slurry was unsuccessful due to the high conductivity of the solution saturating the lock-in amplifiers current input. The use of an impedance analyser to find an appropriate excitation frequency for the lockin amplifier, denoted by a large change in resistance between a ceramic slurry 
with fibers and one without, would be a logical next step before repeating fiber translation and rotation experiments. 


\section{Bibliography}

[1] C. A. Ross, H. I. Smith, T. Savas, M. Schattenburg, M. Farhound, M. Hwang, M. Walsh, M. C. Abraham, and R. J. Ram, "Fabrication of patterned media for high density magnetic storage," Journal of Vacuum science, vol. 17, no. 6, pp. 3168-3176, 1999.

[2] M. Mirza, Q. Liu, T. Yasin, X. Qi, J. Li, and M. Ikram, "Dice-and-fill processing an charactorisation of microscale and high-aspect-ratio $(\mathrm{K}$, $\mathrm{Na}$ )NnO3-based 1-3 lead-free piezoelectric composites," Ceramics International, vol. 42, 032016.

[3] K. Luo, K.-B. Jeong, S.-M. You, D.-H. Lee, J.-Y. Jung, and Y.-R. Kim, "Surface-engineered starch magnetic microparticles for highly effective separation of a broad range of bacteria," ACS sustainable Chemistry \& Engineering, vol. 6, no. 10, pp. 13524-13531, 2018.

[4] H. Chiriac, T. Óvaŕi, and A. Zhukov, "Magnetoelastic anisotropy of amorphous microwires," Journal of Magnetism and Magnetic Materials, vol. 254255, pp. 469-471, 2003.

[5] J. Velázquez, M. Vázquez, and A. Zhukov, "Magnetoelastic anisotropy distribution in glass-coated microwires," Journal of Materials Research, vol. 11, no. 10, pp. 2499-2505, 1996.

[6] P. Marín, D. Cortina, and A. Hernando, "Electromagnetic wave absorbing material based on magnetic microwires," IEEE Transactions on Magnetics, no. 11, pp. 3934-3937, 2008. 
[7] H. Montiel, G. Alvarez, M. Gutierrez, R. Zamorano, and R. Valenzuela, "The effect of metal-to-glass ratio on the low-field microwave absorption at $9.4 \mathrm{GHz}$ of glass-coated CoFeBSi microwires," IEEE Transactions on Magnetics, vol. 42, no. 10, pp. 3380-3382, 2006.

[8] A. D. Kent and D. C. Worledge, "A new spin on magnetic memories," Nature Nanotechnology, vol. 10, 2015.

[9] "2016 endeavour round successful proposals." https:/ / www.mbie.govt.nz/assets / f388f30305/endeavour-fund2016-successful-proposals-detailed-summary.pdf?fbclid=IwAR21Y WvohTqN0BmH9FdIoqQVoXBeuUF6iocQf1h4QVGEWCG8nl-A. Accessed: 2018-06-29.

[10] A. Puttmer, P. Hauptmann, R. Lucklum, O. Krause, and B. Henning, "Spice model for lossy piezoceramic transducers," IEEE Transactions on Ultrasonics, Ferroelectrics, and Frequency Control, vol. 44, pp. 60-66, Jan 1997.

[11] V. F. Janas and A. Safari, "Overview of fine-scale piezoelectric ceramic/polymer composite processing," Journal of the American Ceramic Society, vol. 78, no. 11, pp. 2945-2955, 1995.

[12] M. S. Mirza, Q. Liu, T. Yasin, X. Qi, J.-F. Li, and M. Ikram, “Dice-and-fill processing and characterization of microscale and high-aspect-ratio $(k$, na)nbo3-based 1-3 lead-free piezoelectric composites," Ceramics International, vol. 42, no. 9, pp. 10745 - 10750, 2016.

[13] B. Michaud, E. Beaugnon, A. Sulpice, R. Tournier, and J. Claverie, "Experimental study of the magnetic orientation of ceramic fibers," Materials Transactions, JIM, vol. 41, no. 8, pp. 962-965, 2000.

[14] T. Takahashi, T. Murayama, A. Higuchi, H. Awano, and K. Yonetake, "Aligning vapor-grown carbon fibers in polydimethylsiloxane using dc electric or magnetic field," Carbon, vol. 44, no. 7, pp. 1180 -1188, 2006. 
[15] J. L. Cortes, B. Pire, and J. P. Ralston, "Measuring the transverse polarization of quarks in the proton," Zeitschrift für Physik C Particles and Fields, vol. 55, no. 3, pp. 409-416, 1992.

[16] J. Schmiedmayer, P. Riehs, J. A. Harvey, and N. W. Hill, "Measurement of the electric polarizability of the neutron," Phys. Rev. Lett, vol. 66, pp. 10151018, 1991.

[17] W. Pauli, Writings on Physics and Philosophy, ch. Exclusion Principle and Quantum Mechanics. Heidelberg, Berlin: Springer, 1994.

[18] J. M. D. Coey, Magnetism and Magnetic Materials. Cambrige University Press, 2010.

[19] M. D. Simon and A. K. Geim, "Diamagnetic levitation: Flying frogs and floating magnets (invited)," Journal of Applied Physics, vol. 87, no. 9, pp. 6200-6204, 2000.

[20] P. S. Aithal and H. J. Ravindra, Textbook of Engineering Physics. New Delhi: ACME Learning Private Limited, 1 ed., 2011.

[21] A. Abragam and B. Bleaney, Electron Paramagnetic Resonance of Transition Ions. Oxford Classic Texts in the Physical Sciences, OUP Oxford, 2012.

[22] P. W. Anderson, "Model for the electronic structure of amorphous semiconductors," Physics Review Letters, vol. 34, pp. 953-955, 1975.

[23] E. G. Batyev, "Pauli paramagnetism and Landau diamagnetism," PhysicsUspekhi, vol. 52, no. 12, pp. 1245-1246, 2009.

[24] J. K. Burdett and T. A. McCormick, "Electron localization in molecules and solids: The meaning of ELF," Journal of Physical Chemistry A, vol. 102, no. 31, pp. 6366-6372, 1998.

[25] R. M. Bozorth, Ferromagnetism. Wiley, Aug. 1993. 
[26] S. Chikazumi and C. Graham, Physics of Ferromagnetism 2e. OUP Oxford, 2009.

[27] J. Russell and R. Cohn, Superparamagnetism. Book on Demand, 2012.

[28] A.-F. Ngomsik, A. Bee, M. Draye, G. Cote, and V. Cabuil, “Magnetic nanoand microparticles for metal removal and environmental applications: a review," Comptes Rendes Chimie, vol. 8, no. 6, pp. 963-970, 2005.

[29] S. Laurent, S. Dutz, U. O. Hafeli, and M. Mahmoudi, "Magnetic fluid hyperthermia: Focus on superparamagnetic iron oxide nanoparticles," Advances in Colloid and Interface Science, vol. 166, no. 1, pp. 8-23, 2011.

[30] J. D. Chung and M. Kaviany, "Effects of phonon pore scattering and pore randomness on effective conductivity of porous silicon," International Journal of Heat and Mass Transfer, vol. 43, pp. 521-538, 2000.

[31] J. P. Liu, E. Fullerton, O. Gutfleisch, and D. J. Sellmyer, Nanoscale Magnetic Materials and Applications. Springer, 2009.

[32] D. D. L. Chung, "Electromagneic interference shielding effectiveness of carbon materials," Carbon, vol. 39, no. 2, pp. 279-285, 2001.

[33] X. F. Zhang, X. L. Dong, H. Huang, B. Lv, J. P. Lei, and C. J. Choi, "Microstructure and microwave absorption properties of carbon-coated iron nanocapsules," Journal of Physics D: Applide Physics, vol. 40, no. 17, p. 5383, 2007.

[34] R. Prasher, "Thermal conductivity of composites of aligned nanoscale and microscale wires and pores," Journal of Applied Physics, vol. 100, no. 3, p. 034307, 2006.

[35] T. M. Kuzay, "Cryogenic cooling of x-ray crystals using a porous matrix," Review of Scientific Instruments, vol. 63, no. 1, pp. 468-472, 1992. 
[36] A. Delan, M. Rennau, S. Schulz, and T. Gessner, "Thermal conductivity of ultra low-k dielectrics," Microelectronic Engineering, vol. 70, no. 2, pp. 280 -284, 2003. Materials for Advanced Metallization 2003.

[37] R. Yang and G. Chen, "Thermal conductivity modeling of periodic twodimensional nanocomposites," Phys. Rev. B, vol. 69, p. 195316, May 2004.

[38] T. C. Harman, P. J. Taylor, M. P. Walsh, and B. E. LaForge, “Quantum dot superlattice thermoelectric materials and devices," Science, vol. 297, no. 5590, pp. 2229-2232, 2002.

[39] R. Venkatasubramanian, E. Siivola, T. Colpitts, and B. O'Quinn, “Thinfilm thermoelectric devices with high room-temperature figures of merit," Nature, vol. 413, pp. 597-602, 2001.

[40] E. T. Thostenson and T.-W. Chou, "Aligned multi-walled carbon nanotube-reinforced composites: processing and mechanical characterization," Journal of Physics D: Applied Physics, vol. 35, no. 16, p. L77, 2002.

[41] T. Kimura, H. Ago, M. Tobita, S. Ohshima, M. Kyotani, and M. Yumura, "Polymer composites of carbon nanotubes aligned by a magnetic field," Advanced Materials, vol. 14, no. 19, pp. 1380-1383, 2002.

[42] J. Billaud, F. Bouville, T. Magrini, C. Villevieille, and A. R. Studart, “Magnetically aligned graphite electrodes for high-rate performance Li-ion batteries," Nature Energy, vol. 1, 2016.

[43] A. S. Lübbe, C. Alexiou, and C. Bergemann, "Clinical applications of magnetic drug targeting," Journal of Surgical Research, vol. 95, pp. 200-206, 2001.

[44] R. S. Tade, M. P. More, V. K. Chatap, P. O. Patil, and P. K. Deshmukh, “Fabrication and in vitro drug release characteristics of magnetic nanocellulose fiber composites for efficient delivery of nystatin," Materials Research Express, vol. 5, no. 11, pp. 102-116, 2018. 
[45] N. Kumar, S. Tyeb, N. Manzar, L. Behera, B. Ateeq, and V. Verma, "Entropically driven controlled release of paclitaxel from poly(2-ethyl2-oxazoline) coated maghemite nanostructures for magnetically guided cancer therapy," Soft Matter, vol. 14, pp. 6537-6553, 2018.

[46] A. R. O. Rodrigues, B. G. Almeida, J. P. Araújo, M.-J. R. Queiroz, P. J. Coutinho, and E. M. Castanheira, "Chapter 11 - Magnetoliposomes for dual cancer therapy," in Inorganic Frameworks as Smart Nanomedicines (A. M. Grumezescu, ed.), pp. 489-527, William Andrew Publishing, 2018.

[47] G. Smith, "The development of a low-cost microfludic magnetic separation system," Master's thesis, Victoria University of Wellington, 2009.

[48] M. Scanziani and M. Häusser, "Electrophysiology in the age of light," $N a-$ ture, vol. 461, pp. 930-939, 2009.

[49] E. Blessing, M. D. Esler, and D. P. F. andR oland E. Schmieder, "Cardiac ablation and renal denervation systems have distinct purposes and different technical requirements," Journal of the American College of Cardiology, vol. 6, no. 3, 2013.

[50] M. N. Faddis, J. Chen, J. Osborn, M. Talcott, M. E. Cain, and B. D. Lindsay, "Magnetic guidance system for cardiac electrophysiology: a prospective trial of safety and efficacy in humans," Journal of the American College of Cardiology, vol. 42, no. 11, pp. 1952-1958, 2003.

[51] M. H. Pablico-Lansigan, S. F. Situ, and A. C. S. Samia, “Magnetic particle imaging: advancements and perspectives for real-time in vivo monitoring and image-guided therapy," Nanoscale, vol. 5, pp. 4040-4055, 2013.

[52] K. M. Krishnan, "Biomedical nanomagnetics: A spin through possibilities in imaging, diagnostics, and therapy," IEEE Transactions on Magnetics, vol. 46, pp. 2523-2558, July 2010.

[53] J. Borgert, J. D. Schmidt, I. Schmale, J. Rahmer, C. Bontus, B. Gleich, B. David, R. Eckart, O. Woywode, J. Weizenecker, J. Schnorr, M. Taupitz, 
J. Haegele, F. M. Vogt, and J. Barkhausen, "Fundamentals and applications of magnetic particle imaging," Journal of Cardiovascular Computed Tomography, vol. 6, no. 3, pp. 149 - 153, 2012.

[54] P. W. Goodwill, K. Lu, B. Zheng, and S. M. Conolly, "An x-space magnetic particle imaging scanner," Review of Scientific Instruments, vol. 83, no. 3, p. 033708, 2012.

[55] T. Knopp, T. F. Sattel, S. Biederer, J. Rahmer, J. Weizenecker, B. Gleich, J. Borgert, and T. M. Buzug, "Model-based reconstruction for magnetic particle imaging," IEEE Transactions on Medical Imaging, vol. 29, pp. 1218, Jan 2010.

[56] T. Knopp and T. M. Buzug, Magnetic Particle Imaging: An Introduction to Imaging Principles and Scanner Instrumentation. Springer, 2012.

[57] T. F. Sattel, T. Knopp, S. Biederer, B. Gleich, J. Weizenecker, J. Borgert, and T. M. Buzug, "Single-sided device for magnetic particle imaging," Journal of Physics D: Applied Physics, vol. 42, no. 2, p. 022001, 2009.

[58] T. Le, X. Zhang, A. K. Hoshiar, and J. Yoon, “An electromagnetic navigation system with real-time $2 \mathrm{~d}$ magnetic particle imaging for targeted drug delivery," 2017 IEEE/RSJ International Conference on Intelligent Robots and Systems (IROS), pp. 1895-1900, 2017.

[59] A. V. Abelev, P. J. Valent, and K. T. Holland, "Behavior of a large cylinder in free-fall through water," IEEE Journal of Oceanic Engineering, vol. 32, pp. 10-20, Jan 2007.

[60] J. Newman and K. (Firm), Marine Hydrodynamics. The MIT Press, MIT Press, 1977.

[61] J. Turner and J. Turner, Buoyancy Effects in Fluids. Cambridge Monographs on Mechanics, Cambridge University Press, 1979. 
[62] L. Krysac, Gravitational, Electric, and Magnetic Forces: An Anthology of Current Thought. Contemporary discourse in the field of physics, Rosen Publishing Group, 2006.

[63] J. Svoboda, Magnetic Techniques for the Treatment of Materials. Advances in Global Change Research Series, Springer Netherlands, 2007.

[64] T. Speed and T. Hida, Brownian Motion. Stochastic Modelling and Applied Probability, Springer New York, 2012.

[65] E. Riande, R. Diaz-Calleja, M. Prolongo, R. Masegosa, and C. Salom, Polymer Viscoelasticity: Stress and Strain in Practice. Plastics Engineering, Taylor \& Francis, 1999.

[66] V. Timbrell, "Alignment of carbon and other man-made fibers by magnetic fields," Journal of Applied Physics, vol. 43, no. 11, pp. 4839-4840, 1972.

[67] R. M. Erb, R. Libanori, N. Rothfuchs, and A. R. Studart, "Composites reinforced in three dimensions by using low magnetic fields," Science, vol. 335, no. 6065, pp. 199-204, 2012.

[68] A. D. Shine and R. C. Armstrong, "Experimental studies of suspended ferromagnetic fibers in a magnetic field," Rheologica Acta, vol. 26, pp. 162171, Mar 1987.

[69] T. Kimura, M. Yamato, W. Koshimizu, M. Koike, and T. Kawai, "Magnetic orientation of polymer fibers in suspension," American Chemical Society, vol. 16, no. 2, pp. 858-861, 2000.

[70] J. Ciambella, D. C. Stanier, and S. S. Rahatekar, "Magnetic alignment of short carbon fibres in curing composites," Composites Part B: Engineering, vol. 109, pp. 129 - 137, 2017.

[71] S. E. Spagnolie, "Dropping slender-body theory into the mud," Journal of Fluid Mechanics, vol. 862, p. 1-4, 2019. 
[72] S. B. Lindström and T. Uesaka, "Simulation of the motion of flexible fibers in viscous fluid flow," Physics of Fluids, vol. 19, no. 11, p. 113307, 2007.

[73] C. Marchioli and A. Soldati, "Rotation statistics of fibers in wall shear turbulence," Acta Mechanica, vol. 224, pp. 2311-2329, Oct 2013.

[74] J. Férec and G. Ausias, " 4 - rheological modeling of non-dilute rod suspensions," in Rheology of Non-Spherical Particle Suspensions (F. Chinesta and G. Ausias, eds.), pp. 77 - 117, Elsevier, 2015.

[75] G. K. Batchelor, "The stress generated in a non-dilute suspension of elongated particles by pure straining motion," Journal of Fluid Mechanics, vol. 46, no. 4, p. 813-829, 1971.

[76] J. Evans, The flow of a suspension of force-free rigid rods in a Newtonian fluid. $\mathrm{PhD}$ thesis, University of Cambridge, 1975.

[77] N. Sinai Borker and D. L. Koch, "Slender body theory for particles with non-circular cross-sections," 092018.

[78] İ. Özdemir, "Resistive force theory-based analysis of magnetically driven slender flexible micro-swimmers," Acta Mechanica, vol. 228, pp. 30953109, Sep 2017.

[79] T. Zhang and D. I. Goldman, "The effectiveness of resistive force theory in granular locomotion," Physics of Fluids, vol. 26, no. 10, p. 101308, 2014.

[80] H. Stang and S. P. Shah, "Failure of fibre-reinforced composites by pullout fracture," Journal of Materials Science, vol. 21, pp. 953-957, Mar 1986.

[81] S.-Y. Fu and B. Lauke, "Effects of fiber length and fiber orientation distributions on the tensile strength of short-fiber-reinforced polymers," Composites Science and Technology, vol. 56, no. 10, pp. 1179 - 1190, 1996.

[82] J. Férec, G. Ausias, M. C. Heuzey, and P. J. Carreau, "Modeling fiber interactions in semiconcentrated fiber suspensions," Journal of Rheology, vol. 53, no. 1, pp. 49-72, 2009. 
[83] F. Folgar and I. Charles L. Tucker, "Orientation behavior of fibers in concentrated suspensions," Journal of Reinforced Plastics and Composites, vol. 3, no. 2, pp. 98-119, 1984.

[84] A. V. Abelev, P. J. Valent, and K. T. Holland, "Behaviour of a large cylinder in free-fall through water," Ieee Journal of Oceanic Engineering, vol. 32, no. 1, pp. 10-20, 2007.

[85] G. Bragg, L. van Zuiden, and C. Hermance, "The free fall of cylinders at intermediate reynold's numbers," Atmospheric Environment, vol. 8, no. 7, pp. $755-764,1974$.

[86] R. Chhabra, "Settling on cylinders in power-law liquids," Canadian Journal of Chemical Engineering, vol. 70, pp. 385-386, 041992.

[87] V. D. Azzi and S. W. Tsai, "Anisotropic strength of composites," Experimental Mechanics, vol. 5, pp. 283-288, Sep 1965.

[88] M. Gupta and K. K. Wang, "Fiber orientation and mechanical properties of short-fiber-reinforced injection-molded composites: Simulated and experimental results," Polymer Composites, vol. 14, no. 5, pp. 367-382, 1993.

[89] D. Losic and A. Santos, Nanoporous Alumina: Fabrication, Structure, Properties and Applications. Springer Series in Materials Science, Springer International Publishing, 2015.

[90] K. Maca, P. Dobsak, and A. Boccaccini, "Fabrication of graded porous ceramics using alumina-carbon powder mixtures," Ceramics International, vol. 27, no. 5, pp. $577-584,2001$.

[91] P. Stamenov and J. Coey, "Magnetic susceptibility of carbon-experiment and theory," Journal of Magnetism and Magnetic Materials, vol. 290-291, pp. 279 - 285, 2005. Proceedings of the Joint European Magnetic Symposia (JEMS' 04). 
[92] Y. Yang, B. Zhang, W. Xu, Y. Shi, Z. Jiang, N. Zhou, B. Gu, and H. Lu, "Preparation and properties of a novel iron-coated carbon fiber," Journal of Magnetism and Magnetic Materials, vol. 256, no. 1, pp. 129 - 132, 2003.

[93] M. A. Correa-Duarte, M. Grzelczak, V. Salgueiriño-Maceira, M. Giersig, L. M. Liz-Marzán, M. Farle, K. Sierazdki, and R. Diaz, “Alignment of carbon nanotubes under low magnetic fields through attachment of magnetic nanoparticles," The Journal of Physical Chemistry B, vol. 109, no. 41, pp. 19060-19063, 2005.

[94] A. K. Bentley, J. S. Trethewey, A. B. Ellis, and W. C. Crone, "Magnetic manipulation of copper-tin nanowires capped with nickel ends," Nano Letters, vol. 4, no. 3, pp. 487-490, 2004.

[95] G. Korneva, H. Ye, Y. Gogotsi, D. Halverson, G. Friedman, J.-C. Bradley, and K. G. Kornev, "Carbon nanotubes loaded with magnetic particles," Nano Letters, vol. 5, no. 5, pp. 879-884, 2005.

[96] A. M. James and M. P. Lord, "Macmillan's chemical and physical data," Biochemical Education, vol. 21, no. 2, pp. 109-110, 1993.

[97] "Conductive Composites: Enabling Conductivity." http://www . conductivecomposistes. com. Accessed: 2019-02-12.

[98] "Conductive Composites: Nickel coated carbon fibers." http: / / www . conductivecomposistes.com/pdf/Fibers_overview.pdf. Accessed: 2019-02-12.

[99] J. Crangle and G. M. Goodman, "The magnetization of pure iron and nickel," Proc. Roy. Soc. Lond. A., vol. 321, pp. 477-, 031971.

[100] E. Nieto, J. F. Fernandez, C. Moure, and P. Duran, “Multilayer piezoelectric devices based on pzt," Journal of Materials Science: Materials in Electronics, vol. 7, pp. 55-60, Feb 1996. 
[101] S. Alkoy, "Fabrication and properties of thin-shell monolithic piezoelectric ceramic transducers," Journal of Materials Science, vol. 42, pp. 67426748, Aug 2007.

[102] D. Guo, K. Cai, L. Li, C. Nan, and Z. Gui, "Gelcasting of pzt," Ceramics International, vol. 29, no. 4, pp. 403 - 406, 2003.

[103] K. Al-Malah, "Rheological properties of carbomer dispersions," Annual Transactions of the Nordic Rheology Society, vol. 14, 012006.

[104] S. Mortazavi, "A comparative study between the strength and duration of mucosa-adhesion of transbuccal carbomer based aqueous gels," Iranian Journal of Pharmaceutical Research, vol. Volume 1, no. Number 1, pp. 7-13, 2010.

[105] "Dispersion techniques for carbopol polymers." https:// www.lubrizol.com/-/media/Lubrizol/Life-Sciences/ Documents/TDS/Dispersion-Techniques-for-Carbopol.pdf. Accessed: 2019-02-14.

[106] B. Barry and M. Meyer, "The rheological properties of carbopol gels i. continuous shear and creep properties of carbopol gels," International Journal of Pharmaceutics, vol. 2, no. 1, pp. $1-25,1979$.

[107] D. Brei, J. Halloran, and W. Miao, "Suspension polymerization casting of lead zirconate titanate, part i: Acrylamide hydrogel system," Journal of Materials Science, vol. 38, 062003.

[108] T. Wang and B. Derby, "Ink-jet printing and sintering of pzt," Journal of the American Ceramic Society, vol. 88, no. 8, pp. 2053-2058, 2005.

[109] J. B. Segur and H. E. Oberstar, "Viscosity of glycerol and its aqueous solutions," Industrial \& Engineering Chemistry, vol. 43, no. 9, pp. 2117-2120, 1951. 
[110] "Physical properties of glycerine and its solutions." https: //www.aciscience.org/docs/Physical_properties_of_ glycerine_and_its_solutions.pdf. Accessed: 2019-02-14.

[111] E. McIntosh and J. Ellis, "A simple model for thermal management in solenoids," Review of scientific instruments, vol. 84, 2013.

[112] D. Brown, “Tracker: Video analysis and modeling tool." https:// physlets.org/tracker/, 2018. Accessed: 2018-10-05.

[113] R. Moskowitz and E. Della Torre, "Theoretical aspects of demagnetization tensors," IEEE Transactions on Magnetics, vol. 2, pp. 739-744, December 1966.

[114] T. Papthanasiou and D. Guell, Flow-Induced Alignment in Composite Materials. Woodhead Publishing Series in Composites Science and Engineering, Elsevier Science, 1997.

[115] L. W. McKeehan, “Electrical resistance of nickel and permalloy wires as affected by longitudinal magnetization and tension," Physical Review PHYS REV X, vol. 36, pp. 948-977, 011930.

[116] H. H. Potter, "On the change of resistance of nickel in a magnetic field," Proceedings of The Royal Society of London. Series A, Containing Papers of A Mathematical and Physical Character (1905-1934), vol. 132, pp. 560-569, 01 1931.

[117] S. Yasseri, "Experiment of free-falling cylinders in water," Underwater Technology, vol. 32, no. 3, pp. 177-191, 2014.

[118] M. Mandø and L. Rosendahl, "On the motion of non-spherical particles at high reynolds number," Powder Technology, vol. 202, no. 1, pp. 1 - 13, 2010.

[119] F. E. Luborsky and C. R. Morelock, "Magnetization reversal of almost perfect whiskers," Journal of Applied Physics, vol. 35, pp. 2055 - 2066, 081964. 
[120] R. G. Cox, "The motion of long slender bodies in a viscous fluid part 1. general theory," Journal of Fluid Mechanics, vol. 44, no. 4, p. 791-810, 1970.

[121] B. A. Boukamp, M. T. Pham, D. H. Blank, and H. J. Bouwmeester, "Ionic and electronic conductivity in lead-zirconate-titanate (pzt)," Solid State Ionics, vol. 170, no. 3, pp. $239-254,2004$. 